\title{
Structural and functional analysis of LIM domain- dependent recruitment of paxillin to focal adhesions
}

\section{Marta Ripamonti}

University of Geneva https://orcid.org/0000-0002-1975-7930

\section{Nicolas Liaudet}

Université de Genève, Faculty of Medicine

Bernhard Wehrle-Haller ( $\nabla$ Bernhard.Wehrle-Haller@unige.ch )

University of Geneva https://orcid.org/0000-0002-1159-1147

\section{Article}

Keywords: BiFC, Cross-linking, Dynamics, Fibroblasts, Focal adhesion isolation, Half-life, LD motifs, Nanoscale orientation, Palmitoylation, Photoactivation, Phosphorylation, Plasma membrane, Replacement, Talin, Vinculin

Posted Date: August 21st, 2020

DOl: https://doi.org/10.21203/rs.3.rs-42943/v1

License: (1) (1) This work is licensed under a Creative Commons Attribution 4.0 International License. Read Full License

Version of Record: A version of this preprint was published at Communications Biology on March 29th, 2021. See the published version at https://doi.org/10.1038/s42003-021-01886-9. 
3 Marta Ripamonti ${ }^{1}$, Nicolas Liaudet ${ }^{2}$ and Bernhard Wehrle-Haller ${ }^{1 *}$

${ }^{1}$ Department of Cell Physiology and Metabolism, University of Geneva, Centre Médical Universitaire, Rue Michel-Servet 1, 1211 Geneva 4, Switzerland.

62 Bioimaging Core Facility, Faculty of Medicine, University of Geneva, 1211 Geneva 4, Switzerland.

*Corresponding author, Bernhard.Wehrle-Haller@ unige.ch.

$8 \quad$ Key words

9 BiFC; Cross-linking; Dynamics; Fibroblasts; Focal adhesion isolation; Half-life; LD motifs; 10 Nanoscale orientation; Palmitoylation; Photoactivation; Phosphorylation; Plasma membrane; 11 Replacement; Talin; Vinculin.

\section{Abstract}

13 The LIM domain-dependent localization of the adapter protein paxillin to focal adhesions (FAs) is 14 not mechanistically understood. Here, by combining molecular biology with photoactivation and FA15 isolation experiments, we demonstrate a specific contribution of each LIM domain and reveal the 16 existence of multiple paxillin docking sites in the FA-complex. Mutation of $\beta 3$ integrin at a putative

\section{recruitment of paxillin to focal adhesions}




\section{Introduction}

The anchoring of cells to the extracellular matrix (ECM) is a critical function of integrins. Yet, these heterodimeric receptors are also biomechanical sensors that trigger different signaling pathways in response to extracellular stimuli ${ }^{1,2}$. To accomplish these mechanical and signaling functions, upon ligand binding, integrins cluster in the plasma membrane (PM) and recruit the cytoplasmic adapter proteins talin and kindlin ${ }^{1,3-5}$. Following the mechanical coupling to the actin cytoskeleton, notably through the binding of the $\mathrm{C}$-terminal domain of talin, signaling adapter proteins, such as paxillin and FAK, are recruited to form nascent adhesions ${ }^{6-8}$. Further maturation of these complexes into larger focal adhesions (FAs) occurs in response to additional tension ${ }^{9}$ and recruitment of reinforcing adapter proteins, such as vinculin, that further cross-link talin to the actin cytoskeleton ${ }^{10,11}$.

Paxillin, contains in its N-terminal domain a proline-rich SH3-binding site ${ }^{12}$ and five LD motifs, acting as discrete protein-binding domains, responsible for interaction with structural proteins, such as vinculin, talin and the ILK/Pinch/parvin complex ${ }^{10,13-16}$. In addition, the LD motifs bind and recruit signaling components such as kinases (e.g. FAK) and small GTPases to focal adhesions to control the polymerization of the actin cytoskeleton ${ }^{17,18}$. The activity of the LD motifs, and notably the association with FAK and vinculin, is controlled by numerous phosphorylation sites (Tyr, Ser, Thr), which make paxillin an essential hub in the assembly of FAs and a pivotal player in coordinating integrin-downstream signaling pathways ${ }^{10,19,20}$.

On the other hand, the paxillin C-terminus is composed of four highly similar LIM domains, that constitute the FA targeting motif ${ }^{21}$. Each LIM domain consists of two contiguous zinc-fingers, stacked together through a shared hydrophobic core ${ }^{22,23}$, and mediates protein-protein interactions, to create scaffolds for the assembly of multiprotein complexes at defined cellular sites ${ }^{24-29}$. Structural information of individual LIM domains revealed specific sites of interactions, as for example between the ankyrin repeats of ILK and the first LIM domain of Pinch ${ }^{23}$. Although the biochemical and structural nature of most of the proposed LIM domain-mediated interactions is not yet defined and potentially spread over several LIM domains, many proteins containing multiple LIM domains are recruited to FAs under mechanical tension ${ }^{30-32}$. It was therefore postulated that LIM domains could function as tension sensors ${ }^{32}$ and proposed that could act as a localizer, targeting proteins to specific subcellular locations, such as tensioned or injured F-actin networks ${ }^{22,32-34}$. In the case of the paxillin protein, a LIM3-, and partially LIM2-, dependent FA localization was proposed on the basis of truncations and site-specific mutagenesis experiments ${ }^{21}$. Additional work in paxillin- and Hic-5-null embryonic stem cells, further confirmed this notion, proposing that LIM1 and LIM4 only marginally contribute to FA-targeting ${ }^{35}$. Recently, different kindlin-dependent mechanisms of paxillin 
recruitment to FAs were proposed ${ }^{36-38}$. However, in attached talin-deficient cells, paxillin remained diffuse in the cytoplasm ${ }^{39}$, suggesting that kindlin may not be the only determinant for paxillin recruitment to FAs. Some reports have shown a direct binding of LIM domain-containing proteins, such as the four and a half LIM domain protein 2 (FHL-2), to $\beta$ integrin chains ${ }^{40}$. In addition, a point mutation in the $\beta 1$ integrin tail, which mimics the high-affinity talin-binding site naturally present in the alternatively spliced $\beta 1 \mathrm{D}$ integrin, negatively affected the localization of paxillin to adhesions ${ }^{41}$, proposing that paxillin recruitment is also directly controlled by the $\beta$ integrin peptide. This report is consistent with data demonstrating a direct role for the $\mathrm{Tyr}^{747}$ residue, located in the membraneproximal talin-binding NPLY ${ }^{747}$ motif, in controlling paxillin localization to $\beta 3$ integrin complexes 42,43 . Interestingly, modifications of this sequence or nearby residues, affected paxillin binding to adhesions ${ }^{43,44}$. Importantly, even in the presence of functional integrin/talin complexes that induced integrin activation and clustering, paxillin recruitment still required their mechanical linkage to the actin cytoskeleton ${ }^{45,46}$. Remarkably, paxillin displayed a mechanosensitive behavior on stress fibers ${ }^{34}$ and could bind detergent extracted and stretched cytoskeletons, proposing the existence of tensioninduced paxillin-binding sites ${ }^{30}$. Combined with the notion that some LIM domains can bind Tyrcontaining peptide motifs ${ }^{47,48}$ similar to the NPLY sequence of $\beta 3$ integrin, we previously hypothesized that paxillin LIM domains recognize the $\beta 3$ integrin $\mathrm{Y}^{747}$-presenting motif in a tensiondependent manner ${ }^{42}$.

While the central role of paxillin in the signal transduction from the integrin/talin/kindlin complex is well accepted, it is still controversial how it interacts with these proteins and whether it directly binds $\beta$ integrin subunits ${ }^{21,49,50}$. Aiming for a better understanding of the integrin-dependent mechanisms of paxillin recruitment to FAs, we studied the previously reported paxillin-binding defective $\beta 3^{\mathrm{VE} / \mathrm{YA}}$ integrin in more detail ${ }^{43}$. This integrin, which contains the VE high-affinity talin-binding motif from layilin combined with the $\mathrm{Y}^{747} \mathrm{~A}$ mutation, affected $\beta 3$ integrin-dependent spreading on vitronectin and reduced the recruitment of endogenous paxillin to FAs ${ }^{43}$. Here we show that $\beta 3^{\mathrm{VE} / \mathrm{YA}}$ integrincontaining adhesions exhibit remarkable defects of stability, despite almost normal dissociation dynamics of talin and $\beta 3$ integrin. The mechanical coupling of paxillin to such an integrin or the plasma membrane, rescued the FA instability, highlighting a critical role of paxillin in the mechanical stabilization of the integrin/talin linkage. Importantly, LIM domains appeared sufficient for this rescue, suggesting that they are critical structural components of FAs. By elucidating the mechanisms of LIM domain-mediated paxillin recruitment to $\beta 3$ integrin-containing adhesions, we revealed a defined spatial orientation of paxillin LIM domains, in which LIM4 interacts with the plasma membrane. We also demonstrated that upon deletion of LIM3, the strong paxillin recruitment defect is caused by a steric misplacement of LIM1 and LIM2, forming a major FA-binding element. This 
91 proposes a new model in which a defined spacing of the LIM domains, as well as their correct positioning, is critical for reading the presence of mechanical tension occurring in the integrin/talin/kindlin/F-actin linkage.

\section{Results}

Paxillin anchoring to signaling-defective integrins rescues a rapid-sliding adhesion phenotype

To better understand the mechanism of paxillin recruitment to $\beta 3$ integrin-containing focal adhesions, we employed the previously reported $\beta 3$ integrin mutants, $\beta 3^{\mathrm{VE}}$ and $\beta 3^{\mathrm{VE} / \mathrm{YA}}$ (Fig. 1a), known to modulate the binding of talin, and potentially disrupting the paxillin binding site ${ }^{43}$. While the first mutant presents the high-affinity talin-binding motif (VE), the second, additionally contains the $\mathrm{Y}^{745} \mathrm{~A}$ mutation, which created a spreading-incompetent and paxillin-recruitment deficient integrin 43. Nevertheless, prolonged culturing of $\beta 3^{\mathrm{VE} / \mathrm{YA}}$ integrin-expressing NIH-3T3 fibroblasts in the presence of serum allowed spreading, FA assembly and their phenotypic analysis (Fig. 1b). Prominent GFP-positive central adhesions were induced by the high-affinity talin-binding motif $\left(\beta 3^{\mathrm{VE}}\right)$, but lacking in the $\beta 3^{\mathrm{VE} / \mathrm{YA}}$ integrin-expressing cells (Fig. 1b). At the same time, the latter cells presented much thinner and more elongated FAs (Fig. 1b). Moreover, life cell imaging of $\beta 3^{\mathrm{VE} / \mathrm{YA}}$ integrinexpressing cells unveiled a striking FA instability and accelerated retrograde sliding, when compared to $\beta 3^{\mathrm{WT}}$ and $\beta 3^{\mathrm{VE}}$ integrin-containing adhesions (Supplementary Movies 1 and 2). Importantly, this differential FA stability was not linked to altered expression levels of transfected proteins (Fig. 1c). Considering the previously reported diminished recruitment of paxillin to $\beta 3^{\mathrm{VE} / \mathrm{YA}}$ integrin, the enhanced apparent sliding of FAs presenting this receptor was intriguing. In fact, it contradicted current models proposing that an increased paxillin recruitment is associated with enhanced adhesion turnover ${ }^{8,51}$. We therefore assessed paxillin recruitment to $\beta 3^{\mathrm{VE} / \mathrm{YA}}$-adhesions and determine whether this fulfils a critical role in activating the clutch between the substrate-bound integrin and the retrogradely moving F-actin network ${ }^{4}$.

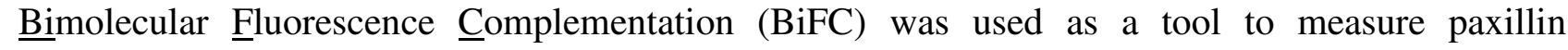
recruitment to $\beta 3$ integrin-positive adhesions in living cells. Accordingly, mCherry_paxillin and $\beta 3^{\mathrm{WT}} / \beta 3^{\mathrm{VE} / \mathrm{YA}}$ integrins C-terminally tagged with complementary citrine fragments were coexpressed, as shown in Fig. 1d. Transfected NIH-3T3 cells, recognized in virtue of their mCherry emission, were imaged via TIRF microscopy, in order to detect the BiFC-derived citrine fluorescence. Although both combinations of proteins generated BiFC (Fig. 1e,f and Supplementary Fig 1a), the VE/YA mutation significantly reduced the efficiency, confirming a diminished recruitment of paxillin 
123 to $\beta 3^{\mathrm{VE} / \mathrm{YA}}$ integrin ${ }^{43}$. However, the generation of BiFC proposed that exogenously expressed paxillin 124 could be transiently recruited to $\beta 3^{\mathrm{VE} / \mathrm{YA}}-\mathrm{FAs}$, potentially relying on endogenous integrins, or 125 interactions with other FA-proteins such as kindlin or talin ${ }^{16,36-38,43}$. Nevertheless, the reduced BiFC 126 efficiency could result from an impaired and very transient paxillin binding to $\beta 3^{\mathrm{VE} / \mathrm{YA}}$-containing 127 adhesions, which reduced the complementation of the citrine fragments ${ }^{52-54}$. Strikingly, the 128 establishment of a BiFC-based mechanical coupling of paxillin to $\beta 3^{\mathrm{VE} / \mathrm{YA}}$ integrin rescued the $\mathrm{FA}$ 129 sliding phenotype (Fig. 1g-h and Supplementary Movie 3). These observations were confirmed by 130 the quantification of the flow of the integrin-associated fluorescence in a frame-by-frame pixel-flux131 based algorithm (Fig. 1i and Supplementary Fig. 1b). Indeed, the VE/YA mutation almost doubled 132 the apparent displacement of integrins per minute $\left(\beta 3^{\mathrm{WT}} 0.21 \mu \mathrm{m} / \mathrm{min}, \beta 3^{\mathrm{VE} / \mathrm{YA}} 0.40 \mu \mathrm{m} / \mathrm{min}\right)$, but, 133 after the BiFC-based trapping of paxillin, the stability was recovered $\left(\beta 3^{\mathrm{VE} / \mathrm{YA}}+\mathrm{BiFC} 0.13 \mu \mathrm{m} / \mathrm{min}\right)$ 134 and even improved compared to $\beta 3^{\mathrm{WT}}$ integrin-containing adhesions. Interestingly, in the presence of 135 the mechanical integrin-paxillin coupling, the displacements of $\beta 3^{\mathrm{WT}}$ and $\beta 3^{\mathrm{VE} / \mathrm{YA}}$ containing FAs 136 were indistinguishable (Fig. 1i). These results propose that the fast retrograde-sliding adhesion 137 phenotype induced by the $\beta 3^{\mathrm{VE} / \mathrm{YA}}$ integrin mutant was linked to a compromised paxillin binding and 138 retention within FAs, possibly impairing the maturation of highly dynamic integrin/talin clusters into 139 mature FAs ${ }^{45}$. 
$3^{\text {WT }} \quad$ WDTANNPLY ${ }^{747}$ KEAT

$\beta 3^{\mathrm{VE}} \quad W--V E N P L Y^{745} \mathrm{KEAT}$

$\beta 3^{\mathrm{VE} / \mathrm{YA}} \mathrm{W}--\mathrm{VENPLA}{ }^{745} \mathrm{KEAT}$

C $\quad \frac{\text { mCh_paxillin + }}{\beta 3^{\text {WT }} \beta 3^{\mathrm{VE}} \beta 3^{\mathrm{VENA}}}$

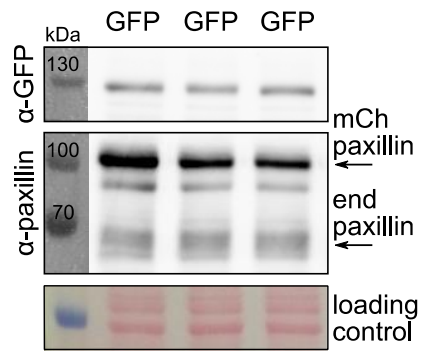

$\beta 3^{\text {WT }}$ GFP $+m$ Ch pax
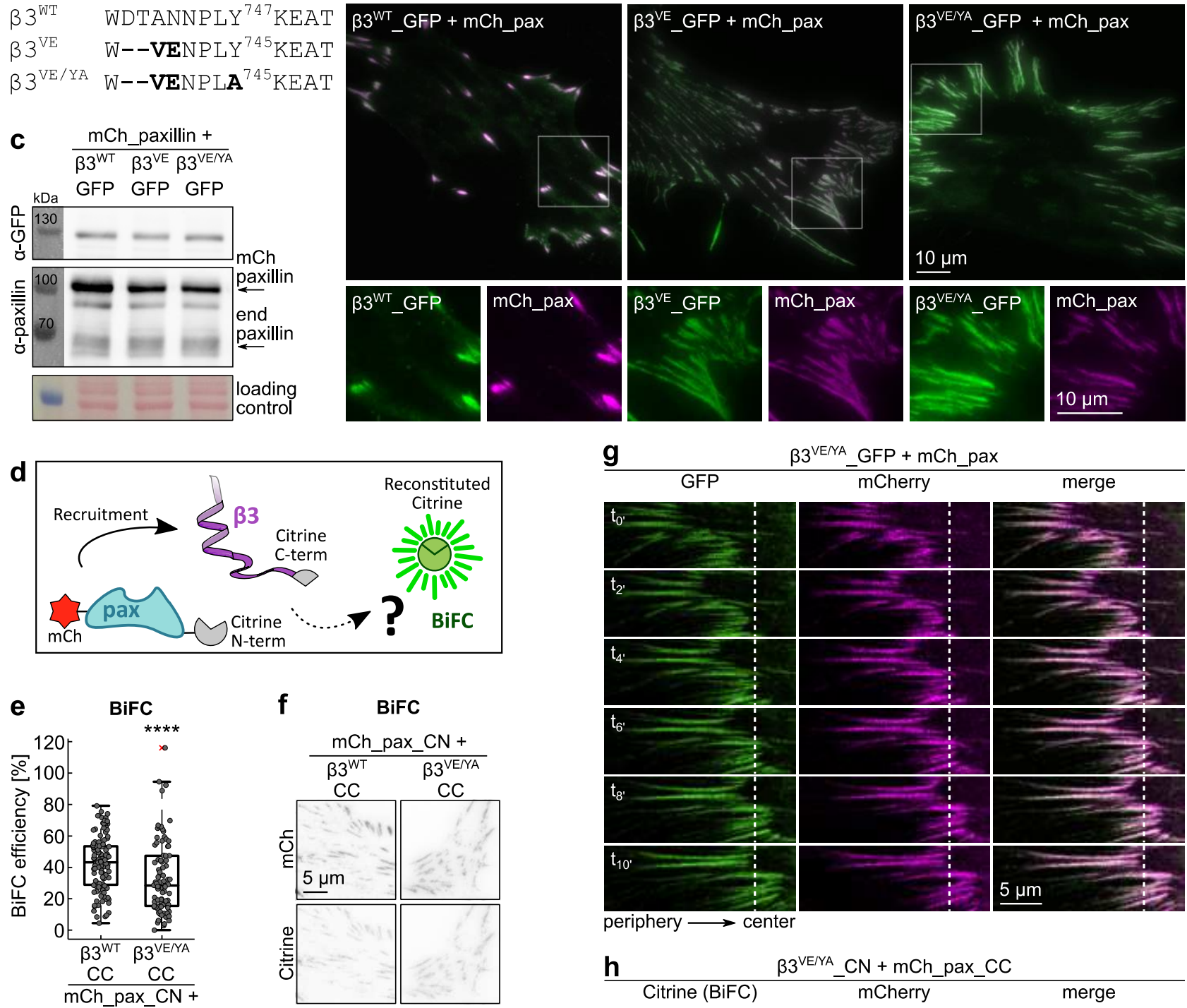

i Integrins flow in adhesions
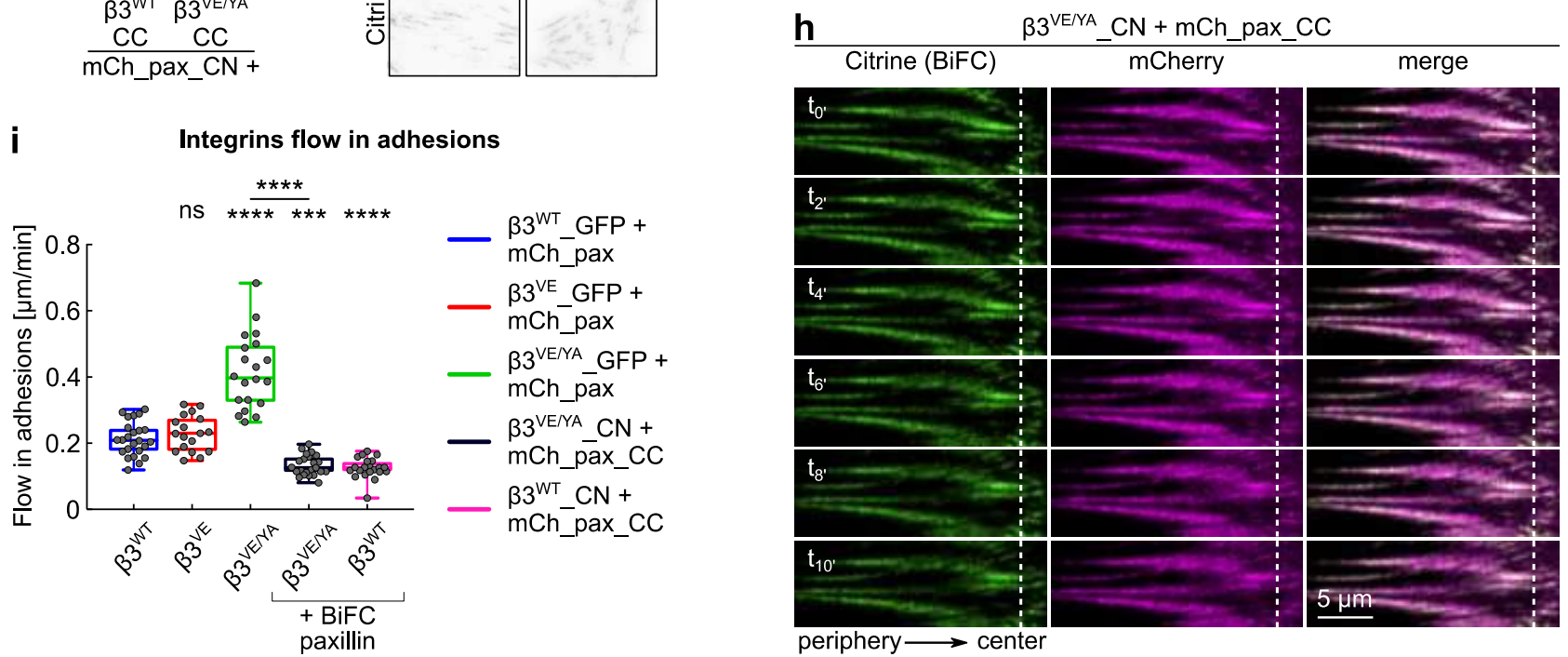

141 Figure 1 - Morphology and dynamics of wild-type and mutant $\beta 3$ GFP integrin-positive adhesions. (a) Sequence alignment of $\beta 3$ integrins, wt and mutants, at the NPLY ${ }^{747}$ motif. The highaffinity talin-binding chimeric $\beta 3$ integrins were obtained by introducing the VE motif from layilin. Bold indicates mutated residues. (b,c) NIH-3T3 cells transiently transfected with $\beta 3$ GFP integrins, wild-type or mutants, and mCherry_paxillin. (b) Representative TIRF images of cells fixed 24 hours after spreading on glass coverslips in serum containing medium and (c) recombinant protein expression assessed by western blotting against GFP and total paxillin. Loading control: Ponceau 
staining. (d) Schematic representation of the BiFC assay used to evaluate paxillin recruitment to $\beta 3$ integrins. (e,f) BiFC upon co-expression of mCherry_paxillin_CN and $\beta 3^{\mathrm{WT}}{ }_{-} \mathrm{CC}$ or $\beta 3^{\mathrm{VE} / \mathrm{YA}}{ }_{-} \mathrm{CC}$ in NIH-3T3 fibroblasts. (e) Quantification of the BiFC efficiency. Statistical analysis is provided in Supplementary Table 1. (f) Representative TIRF images. (g,h) Kymographs representative of the displacement of $\beta 3^{\mathrm{VE} / \mathrm{YA}}$-containing adhesions, over a time period of 10 minutes, in the absence (g) and in the presence $(\mathbf{h})$ of mechanically (BiFC) coupled paxillin. (i) Quantification of the $\beta 3$ integrins flow in adhesions, expressed as mean displacement over time $(\mu \mathrm{m} / \mathrm{min})$ per replicate. Statistical analysis is provided in Supplementary Table 2.

\section{Photoactivation of paxillin reveals its rapid dissociation from $\beta 3^{\mathrm{VE} / \mathrm{YA}}$ integrin-} containing adhesions

Following the hypothesis that impaired paxillin binding caused the instability of $\beta 3^{\mathrm{VE} / \mathrm{YA}}$-positive FAs, paxillin dynamics at adhesion sites were investigated. Its dissociation rate was quantified via photoactivation experiments, and used as a readout of its FA-binding capacity. Given the plausible influence of the VE and VE/YA mutations on the dynamics of talin, and therefore of $\beta 3$ integrin itself ${ }^{45}$, we first studied talin and integrin turnovers in their presence. To do so, $\beta 3$ integrins were $\mathrm{C}$ terminally tagged with the photoactivatable-GFP(PA-GFP), co-expressed with mCherry_paxillin and photoactivated in a single mCherry-positive peripheral FA. The fluorescence loss after photoactivation demonstrated that the high-affinity talin-binding motif (VE) enhanced the stability of the $\beta 3$ integrin, prolonging its half-life $\left(\mathrm{t}_{1} / 2\right)$ in adhesions by 1.4 -fold (median $\mathrm{t}_{1 / 2}$ of $\beta 3^{\mathrm{WT}} 104 \mathrm{sec} v s$ $147.1 \mathrm{sec}$ of $\beta 3^{\mathrm{VE}}$ integrin). However, for the $\beta 3^{\mathrm{VE} / \mathrm{YA}}$ integrin mutant, the dissociation dynamics was increased, and the median of the estimated half-lives was $75.3 \mathrm{sec}$, meaning 0.75 -fold that of the wildtype integrin (Fig. 2a-c and Supplementary Fig. 3d).

To measure the talin off-rate, a similar photoactivation strategy was applied, with the $\beta 3$ integrin carrying the mCherry tag. Coherent with the slower turnover of the $\beta 3^{\mathrm{VE}}$ integrin, the half-life of PAGFP_talin was prolonged in $\beta 3^{\mathrm{VE}}$ - compared to $\beta 3^{\mathrm{WT}}$-positive adhesions $(82.6 \mathrm{sec}$ and $46.7 \mathrm{sec}$, respectively). Talin dissociation from $\beta 3^{\mathrm{VE} / \mathrm{YA}}$ integrin-adhesions $(43.2 \mathrm{sec})$ was instead almost identical to that observed with the wild-type receptor (Fig. 2d-f and Supplementary Fig. 3e). Nevertheless, the ratio between half-lives, revealed a stabilization of talin on the VE-expressing integrins: the half-life of talin in respect to that of wild-type $\beta 3$ integrin was 0.4 , while increased to 0.6 in FAs containing $\beta 3^{\mathrm{VE}}$ or $\beta 3^{\mathrm{VE} / \mathrm{YA}}$ integrins.

Considering the intricate network of paxillin interactions, we assured that the tagging of paxillin did not affect its binding capacity to FA-complexes. The equivalent dissociation rate of recombinant paxillins, presenting the PA-GFP either at the $\mathrm{N}$ - or $\mathrm{C}$-terminus, indicated that the fusion of tags at the extremities did not alter its binding FAs (Supplementary Fig. 3a-c). Surprisingly, the VE motif, which increased the affinity of talin for the $\beta 3$ tail by about 20 -fold ${ }^{43}$ and reduced the dissociation 
183 rate of these two proteins from FAs, caused a faster dissociation of paxillin and a shortening of its

184 half-life by approximately $40 \%$ (paxillin $\mathrm{t}_{1 / 2}$ in $\beta 3^{\mathrm{WT}}$ adhesions $72 \mathrm{sec}$, while in $\beta 3^{\mathrm{VE}}$ adhesions 42.3

$185 \mathrm{sec}$ ) (Fig. 2g-i and Supplementary Fig. 3f). Adding the $\mathrm{Y}^{747} \mathrm{~A}$ substitution into the $\beta 3^{\mathrm{VE}}$ integrin

186 sequence further destabilized paxillin binding to FAs and shortened its half-life to less than 30\% (19.7

$187 \mathrm{sec}$ ) (Fig. 2g-i and Supplementary Fig. 3f). Therefore, the impact of the VE/YA mutation was much

188 greater on the dynamics of paxillin than on talin (almost unaltered between $\beta 3^{\mathrm{VE} / \mathrm{YA}}$ and $\beta 3^{\mathrm{WT}}$

189 adhesions) and $\beta 3$ integrin ( $\mathrm{t}_{1 / 2}$ of $\beta 3^{\mathrm{VE} / \mathrm{YA}}$ reduced of $25 \%$ compared to that of $\beta 3^{\mathrm{WT}}$ ). Importantly,

190 these dynamic measures of paxillin dissociation would propose a highly compromised paxillin

191 binding to $\beta 3^{\mathrm{VE} / \mathrm{YA}}$ adhesions, which might be functionally linked to their rapid-sliding phenotype.

a $\quad \beta 3$ integrins dissociation curves

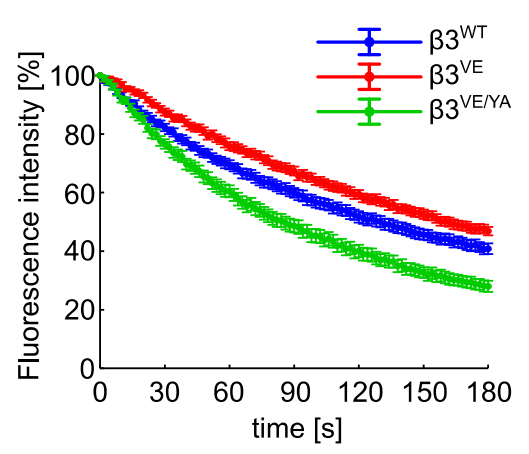

d

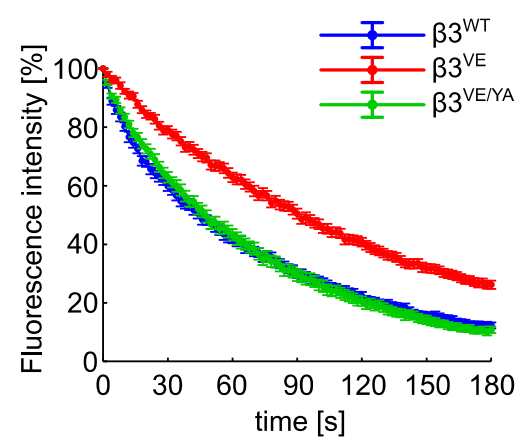

9

Paxillin dissociation curves

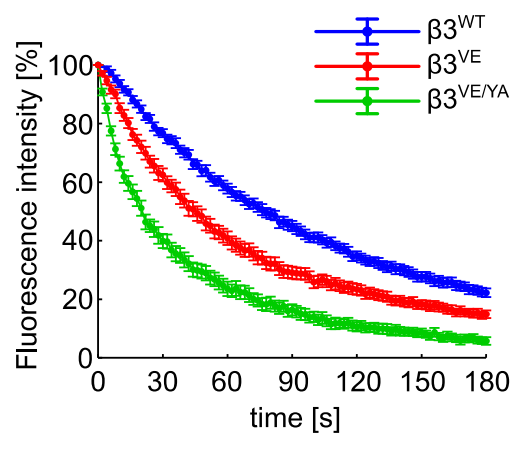

b Integrins half-lives

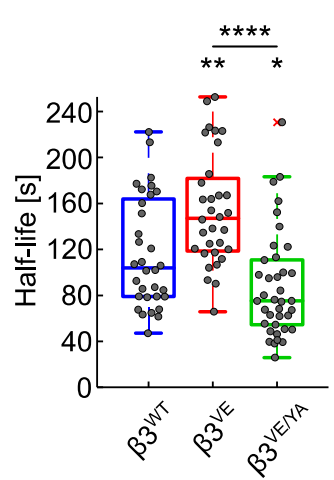

e

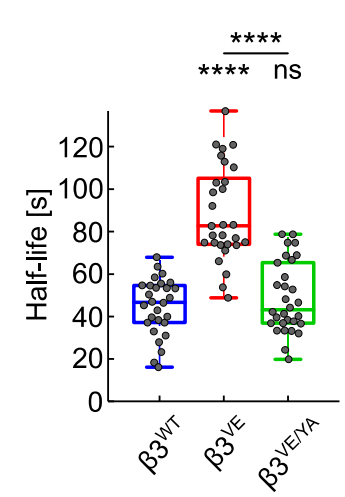

h Paxillin half-life

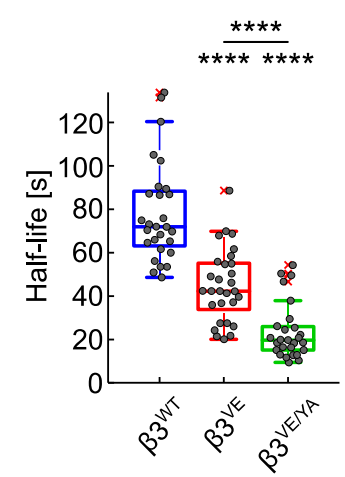

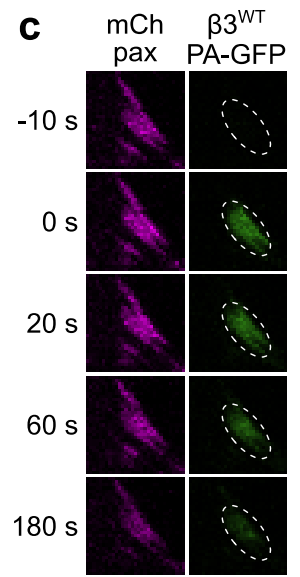
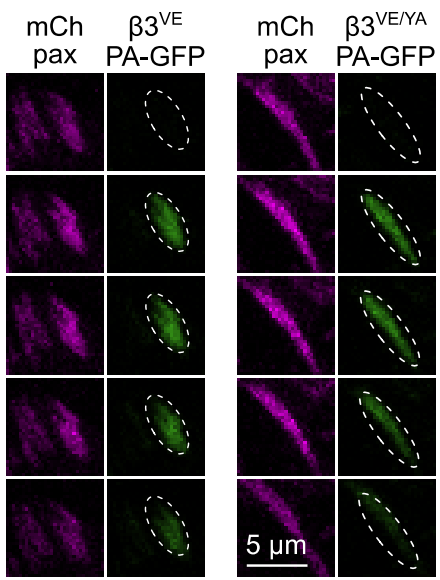

f

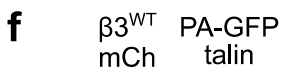

$\begin{array}{lc}\beta 3^{\text {VE }} & \text { PA-GFP } \\ \mathrm{mCh} \text { talin }\end{array}$
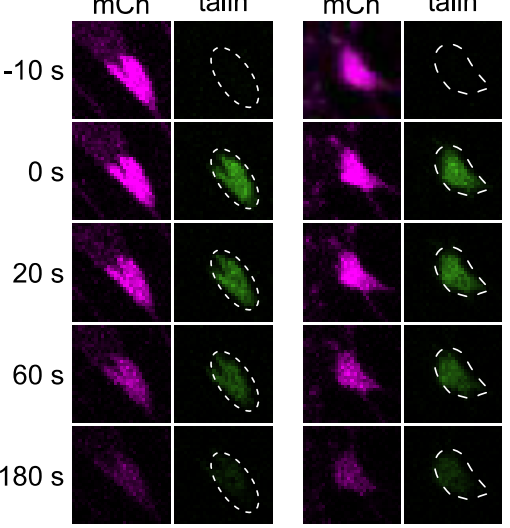

$\beta 3^{\text {VE/YA } P A-G F P ~}$ $\mathrm{mCh}$ talin

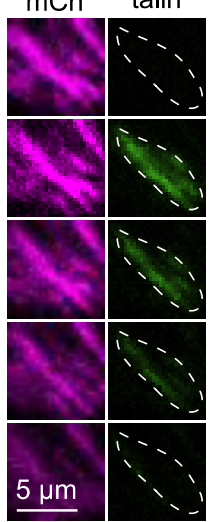

i
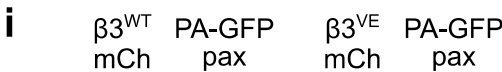

$\beta 3^{\text {VE/YA }}$ PA-GFP
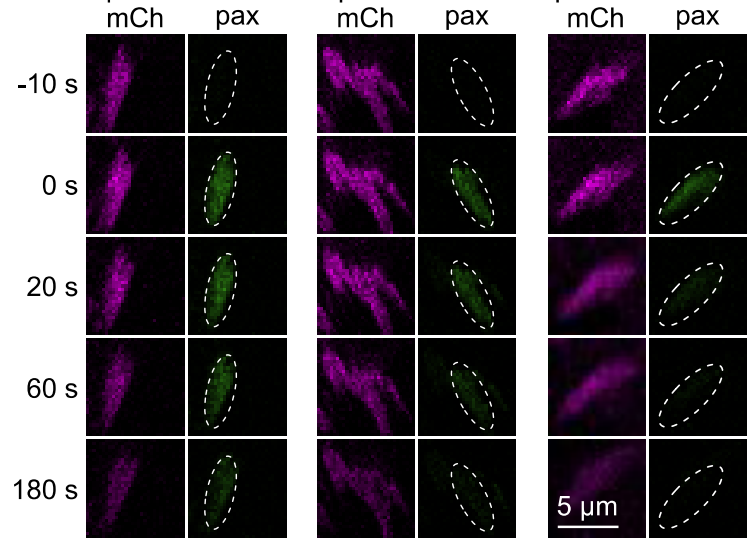
Figure 2 - Analysis of the dissociation dynamics of the $\beta 3$ integrin/talin/paxillin complex. (a,d,g) Experimental dissociation curves of (a) $\beta 3$ _PA-GFP integrins from mCherry_paxillin-positive focal adhesions and of (d) PA-GFP_talin and (g) PA-GFP paxillin from $\beta 3$ mCherry-positive focal adhesions. (b,e,h) Box plot of the half-lives of (b) $\beta 3$ _PA-GFP integrins in mCherry_paxillin-positive FAs and of (e) PA-GFP_talin and (h) PA-GFP_paxillin in $\beta 3$ mCherry-positive FAs. Statistical analysis is provided in Supplementary Table 3. (c,f,i) Representative images of photoactivation time series of experiments in $\mathbf{a}-\mathbf{b}, \mathbf{d}-\mathbf{e}$ and $\mathbf{f}-\mathbf{g}$.

Paxillin dissociation dynamics reflects the mechanical stability of isolated focal adhesions

Since the rapid inward-sliding of $\beta 3^{\mathrm{VE} / \mathrm{YA}}$ integrin-presenting FAs was potentially linked to the fast dissociation of paxillin from them, it appeared relevant to characterize the mechanical stability of the entire FA-complex with an alternative approach. We therefore applied the protocol developed by Kuo et al. to isolate apparently intact FAs, and we revealed their mechanical integrity in respect to the expressed GFP-tagged $\beta 3$ integrin construct. Consistent with the rapid displacement of $\beta 3^{\mathrm{VE} / \mathrm{YA}}$ integrin-containing adhesions, these apparently mechanical fragile structures, did not survive the FAisolation procedure. On the contrary, the shearing of the PM, the washing away of the cytosol with a jet of PBS ${ }^{55}$ and the subsequent fixation, allowed detecting isolated FAs containing $\beta 3^{\mathrm{WT}}$ or $\beta 3^{\mathrm{VE}}$ integrins in virtue of their fluorescence (Fig. 3a,b). Importantly, these isolated adhesive structures were mCherry_paxillin-positive. Reasonably, the dissociation curves of the photoactivation studies reflected the strength of paxillin binding to FAs, which determined its ability to remain associated during the FA-isolation process. Indeed, while in intact cells, paxillin was recruited to $\beta 3^{\mathrm{WT}}$ and $\beta 3^{\mathrm{VE}}$ adhesions equally well (Fig. 1b), in isolated FAs, the expression of the $\beta 3^{\mathrm{VE}}$ integrin variant caused a drop of paxillin/integrin fluorescence ratio of about $50 \%$ (Fig. 3a,b), which was consistent with the accelerated dissociation rate of paxillin from this type of adhesions (Fig. 2g,h). To assure that the mCherry-tag and the exogenous paxillin expression did not affect its mechanical retention in FAs, cells were transfected only with the integrin proteins and the presence of endogenous paxillin in isolated adhesions was verified by immunostaining. As for the mCherry-labelled paxillin, the immunofluorescence signal dropped in adhesions presenting the $\beta 3^{\mathrm{VE}}$, compared to the wild-type integrin (Fig. 3c,d). We also evaluated the binding of vinculin and demonstrated its reduction in $\beta 3^{\mathrm{VE}}$ integrin-containing, compared to $\beta 3^{\mathrm{WT}}$ integrin-, adhesions (Fig. 3e,f). This was unexpected considering the proposed talin-dependent recruitment and binding of vinculin to adhesions and the expression of an integrin with a high-affinity talin-binding site ${ }^{2,4,39,43}$. However, it was consistent with a critical role of paxillin in orchestrating the nanoscale localization of vinculin within FAs ${ }^{10}$. Accordingly, it has been demonstrated that paxillin and vinculin have comparable recovery curves after photobleaching ${ }^{56}$, proposing that the VE mutation loosened vinculin binding to FAs, in a paxillin-dependent manner. These results suggested that, despite a high-affinity talin/integrin 
interaction, a proper paxillin-mediated focal adhesion assembly and maturation is required to firmly

230 anchor cells to the substratum and to create FAs with sufficient mechanical cohesion to survive the 231 isolation protocol.
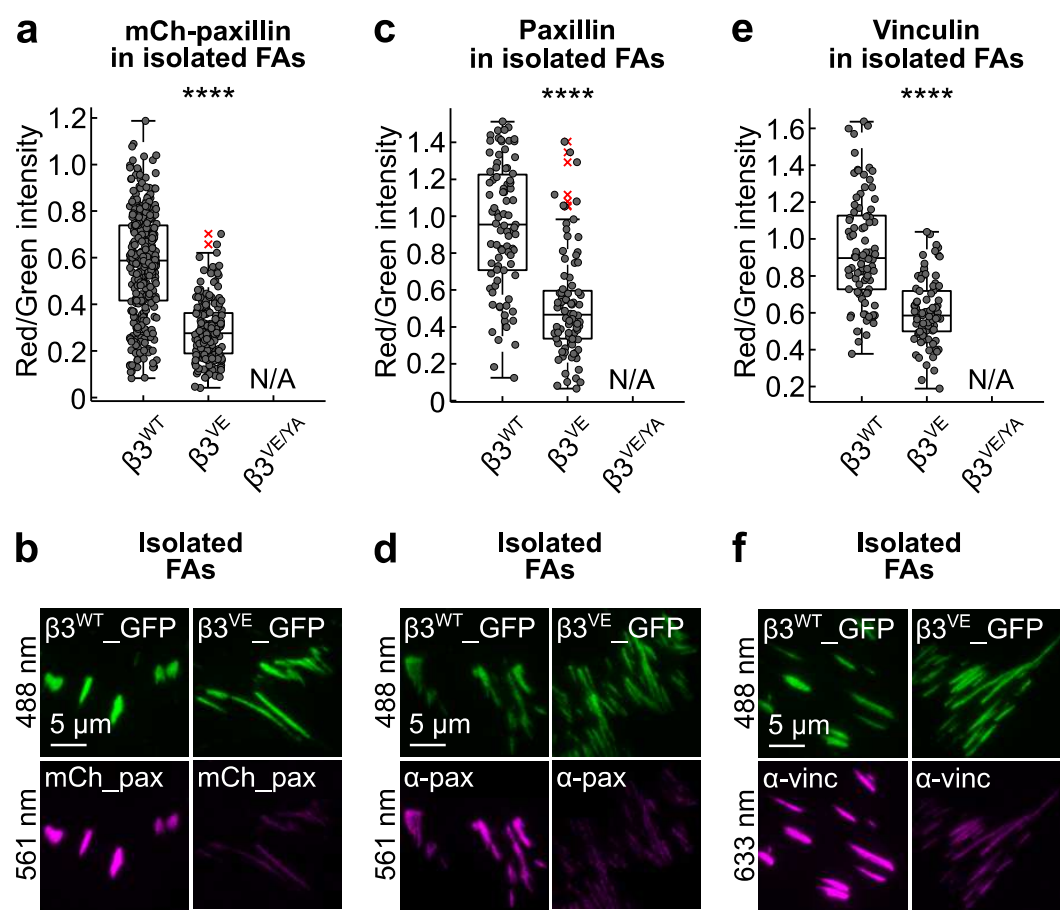

Figure 3 - Analysis of paxillin and vinculin binding upon isolation of FA-complexes. (a,c,e) Quantification of cytoplasmic proteins retained in $\beta 3^{\mathrm{WT}}$ _GFP- or $\beta 3^{\mathrm{VE}}$-GFP-positive FAs, isolated from NIH-3T3 fibroblasts. (a) mCherry_paxillin, (c) endogenous paxillin and (e) endogenous vinculin. Statistical analysis is provided in Supplementary Table 4. (b,d,f) Representative TIRF images of FAs isolated from NIH-3T3 cells of experiments quantified in $\mathbf{a}, \mathbf{c}$ and $\mathbf{e}$.

\section{The N-terminal, LD motif-containing sequence, enhances paxillin stability in FAs}

239 By applying photoactivation and FA-extraction protocols to two paxillin fragments corresponding to 240 the N-terminal LD motif-containing sequence and to the C-terminal LIM domains respectively (Fig. $2414 a)$, we confirmed the previously proposed FA-targeting activity of the second ${ }^{21}$. Although the Nterminal paxillin fragment could be photoactivated and detected in the area corresponding to FAs

243 (Supplementary Fig. 2a), it showed no specific retention and dissociated similarly to the 244 cytoplasmically located PA-GFP control (median half-life of 1.8 and 2 sec, respectively) (Fig. 4b,c 245 and Supplementary Fig. 3g). Accordingly, this paxillin fragment was not detected in isolated 246 adhesions above control levels (Fig. 4d,e). In contrast, the LIM domain-only construct (paxillin C247 terminus) was efficiently photoactivated (Supplementary Fig. 2a), retained in FAs for a prolonged 248 period ( $\mathrm{t}_{1 / 2}$ about $50 \%$ of full-length paxillin) (Fig. 4b,c and Supplementary $3 \mathrm{~g}$ ) and significantly 249 present in isolated FAs (Fig. 4d,e). Despite the evident interaction of the paxillin LIM domain-only protein with FAs, our analyses revealed that it dissociated significantly faster than the full-length 
251 paxillin, and was less retained in isolated FAs (LIMs/integrin fluorescence 59\% in respect to 252 paxillin/integrin). These data, therefore, support an auxiliary function of paxillin N-terminal 253 sequences in stabilizing the LIM domain-dependent localization at FAs.

254 To circumscribe this stabilization activity to a specific paxillin sequence, the dynamic behavior of $\delta$ 255 paxillin, a naturally occurring paxillin isoform missing the first 133 amino acids (Fig. 4f), was 256 investigated. Compared to LIMs-only, $\delta$ paxillin showed an improved stability (median t $1 \frac{2}{2} 35.4 \mathrm{sec}$ 257 and $47.4 \mathrm{sec}$, respectively), but still a dissociation rate significantly faster than that of the canonical 258 paxillin $(72 \mathrm{sec})$, proposing the presence of important residues within the missing amino acids (Fig. $2594 \mathrm{~g}, \mathrm{~h}$ and Supplementary Fig. 2b and 3h). Interestingly, $\delta$ paxillin lacks tyrosines 31 and 118, which 260 phosphorylation enhances FAK and vinculin binding ${ }^{10,14,57}$. When their phosphorylation was 261 prevented by means of site-directed mutagenesis (paxillin $\mathrm{Y}^{31 / 118} \mathrm{~F}$, Fig. 4f), the resulting dissociation 262 curve overlaid that of $\delta$ paxillin. These results are consistent with the hypothesis that paxillin 263 stabilization in adhesion occurs in response to phosphorylation of the $\mathrm{Y}^{31}$ and/or $\mathrm{Y}^{118}$, potentially 264 leading to an exposure of LD motifs that enables a mechanical interaction with other components of 265 FAs, such as talin, FAK, and vinculin ${ }^{10,16,21,58,59}$.

266 Considering this novel stabilizer function of the paxillin $\mathrm{N}$-terminus, we tested if the paxillin267 dependent arrest of $\beta 3^{\mathrm{VE} / \mathrm{YA}}$ integrin-positive FAs relied on its activity. Although the BiFC-based 268 mechanical coupling of $\delta$ paxillin or paxillin $\mathrm{Y}^{31 / 118} \mathrm{~F}$ to the mutant integrin was as efficient as the 269 wild-type protein, the LIM domains alone were, surprisingly, sufficient to arrest the inward flow of $270 \beta 3^{\mathrm{VE} / \mathrm{YA}}$ integrin in adhesion to wild-type levels $\left(\beta 3^{\mathrm{VE} / \mathrm{YA}}+\mathrm{LIMs}\right.$ BiFC $0.20 \mu \mathrm{m} / \mathrm{min}, \beta 3^{\mathrm{WT}}$ without 271 BiFC $0.21 \mu \mathrm{m} / \mathrm{min}$ ) (Fig. 4i). This further corroborated a critical function of the LIM domains in 272 mechanically stabilizing integrin/talin complexes, thus suggests an essential structural role of paxillin 273 in FAs. 


\section{a}

pax

wt

pax

N-term

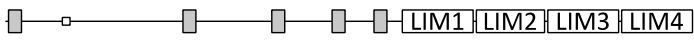

LIMs

\section{-LIM1 LIM2 LIM3 LIM4}

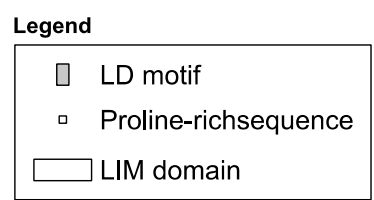

b Paxillins dissociation curves

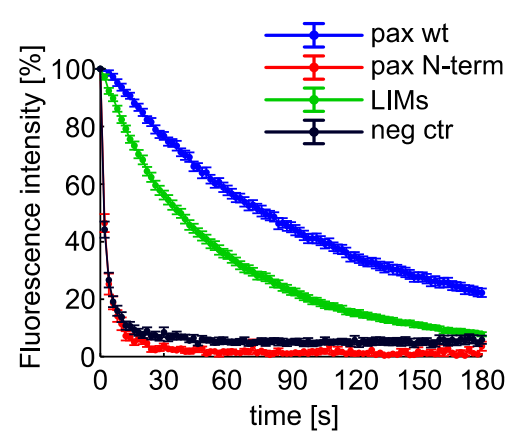

C Paxillins half-lives

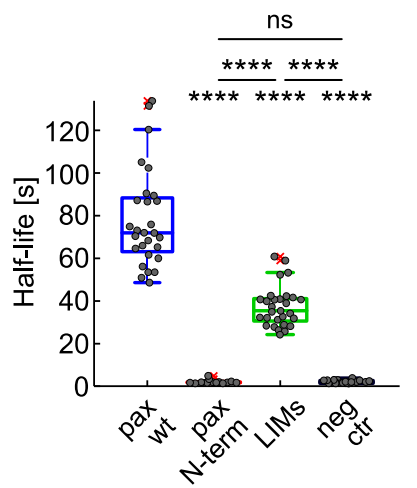

d Paxillins in isolated FAs

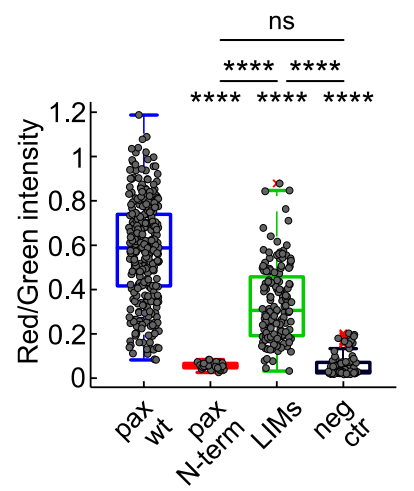

e

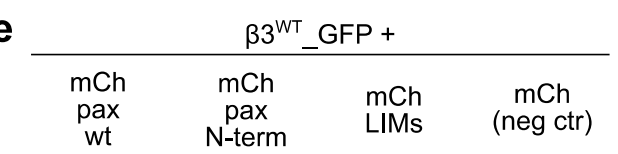

\begin{tabular}{cccc}
\multicolumn{4}{c}{$\beta 3^{\mathrm{WT}} \_\mathrm{GFP}+$} \\
\hline $\begin{array}{c}\mathrm{mCh} \\
\mathrm{pax} \\
\mathrm{wt}\end{array}$ & $\begin{array}{c}\mathrm{mCh} \\
\text { pax } \\
\text { N-term }\end{array}$ & $\begin{array}{c}\mathrm{mCh} \\
\text { LIMs }\end{array}$ & $\begin{array}{c}\mathrm{mCh} \\
\text { (neg ctr) }\end{array}$
\end{tabular}
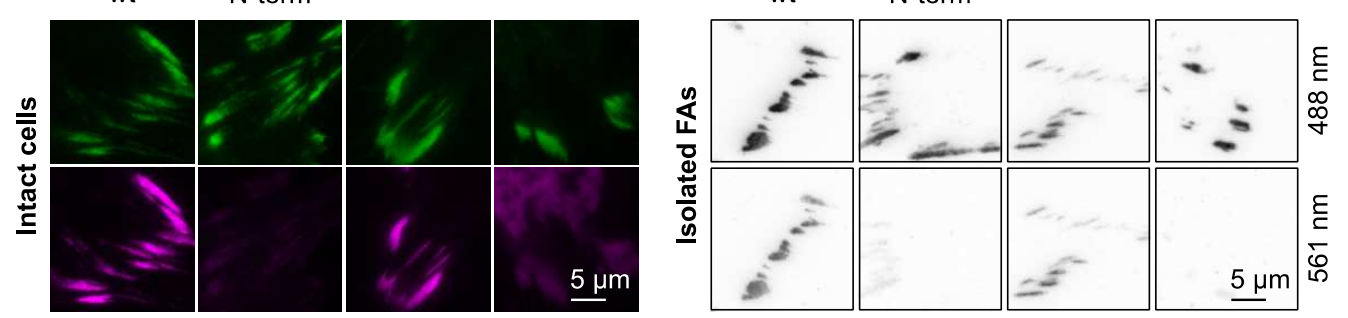

f

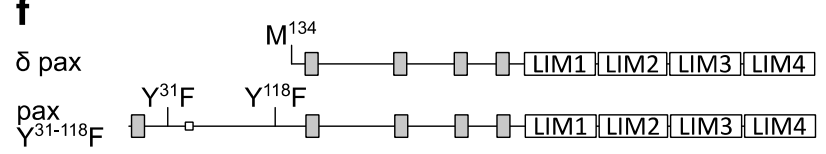

i $\quad \beta 3^{\text {VENA }}$ integrin flow in adhesions

g Paxillins dissociation curves
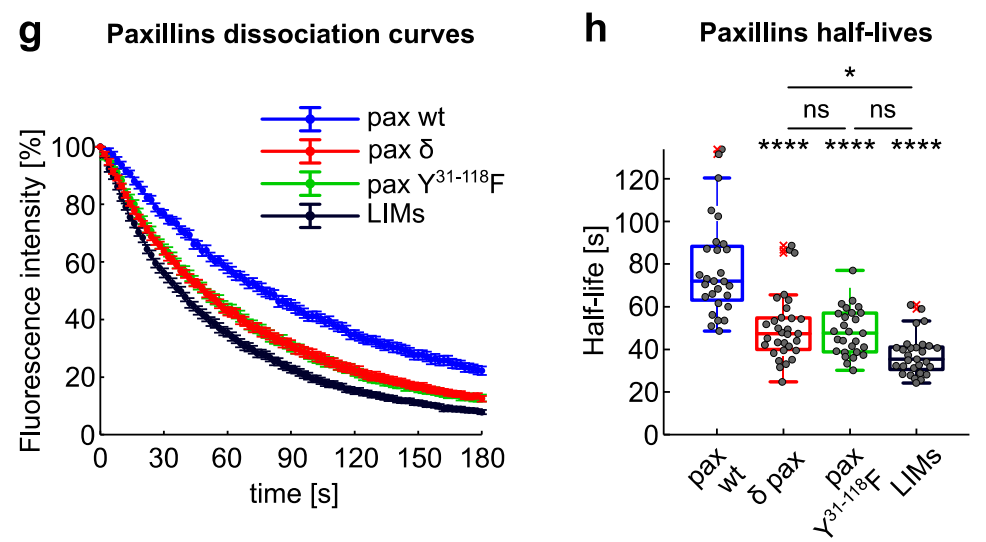

Figure 4 - Paxillin binding to adhesions is driven by the LIM domains but stabilized by the Nterminus. (a,f) Schematic representation of paxillin (a) truncation mutants and (f) N-terminal mutants. All proteins were N-terminally tagged with PA-GFP or mCherry. (b,g) Experimental dissociation curves of PA-GFP_paxillin wt and (b) truncation mutants or (g) $\mathrm{N}$-terminal mutants, from 33 m mCherry-positive FAs. (c,h) Box plot of the half-life of PA-GFP_paxillin wt and (c) 
truncation mutants or (h) $\mathrm{N}$-terminal mutants, in $\beta 3$ mCherry-positive FAs. Statistical analysis is 281 provided in Supplementary Table 3. (d) Quantification of mCherry_paxillin wt and truncation 282 mutants retained in FAs, isolated from NIH-3T3 cells co-expressing $\beta 3^{\mathrm{WT}}$ GFP. Statistical analysis 283 is provided in Supplementary Table 4. (e) Representative TIRF images of FAs in NIH-3T3 cells co284 expressing $\beta 3^{\mathrm{WT}}$ _GFP and mCherry_paxillin proteins. Left panel: FAs in intact cells; right panel: 285 isolated FAs. (i) Quantification of the $\beta 3^{\mathrm{VE} / \mathrm{YA}}$ integrin flow in adhesions, expressed as mean 286 displacement over time $(\mu \mathrm{m} / \mathrm{min})$ per replicate. Statistical analysis is provided in Supplementary 287 Table 2. Neg ctr: PA-GFP in photoactivation experiments and mCherry in isolated FAs.

\section{Differential contribution of LIM domains to paxillin docking to FAs}

289 To precisely assess the contribution of each LIM domain to paxillin docking to FAs, we designed 290 paxillin mutants missing individual LIM domains (Fig. 5a,b and Supplementary Fig. 4a), verified 291 their proper expression (Fig. 5c), and analyzed them by photoactivation and FA-isolation 292 experiments. This first approach unveiled a wide range of paxillin dissociation dynamics (Fig. 5d,e 293 and Supplementary Fig. 2c and 3i), strongly correlating with the quantitative analysis of mechanical 294 retention in isolated FAs (Fig. 5f,g). Specifically, the medians of the dissociation half-lives measured 295 for paxillin- $\Delta$ LIM1, $-\Delta$ LIM2, $-\Delta$ LIM3 and - $\Delta$ LIM4 were respectively $15 \%, 7 \%, 9 \%$ and $42 \%$ of that 296 of paxillin wt (Fig. 5e). Similarly, the residual normalized paxillin-derived fluorescence in isolated 297 adhesions was 14\%, 7\%, 10\% and 25\% compared to that of mCherry_paxillin wt (Fig. 5f). In line 298 with previous qualitative observations ${ }^{21,35}$, the deletion of LIM4 was significantly the least severe, 299 while surprisingly the LIM1 and LIM2 deletions were comparable to that of LIM3, which so far 300 represented the most critical FA-interaction domain. These results suggested the existence of multiple 301 FA-interaction sites, spread along the four LIM domains. Except for LIM4, which removal would not 302 affect the spacing between LIM1-LIM2-LIM3 domains, the outcome of all other LIM-deletions could 303 also reflect the mis-localization of the remaining LIM domains, preventing to identify the specific 304 binding function of the missing LIM domain. 
a

$$
\operatorname{pax} \Delta \mathrm{LIM}
$$

\section{b}

LIM1 G $^{322}$ VCGACKKPIAGQVVTAMGKTWHPEHFVCTHCQEEIGSRNFFERDGQPYCEKDYHNLFS ${ }^{380}$ LIM2 P ${ }^{381}$ RCYYCNGPILDKVVTALDRTWHPEHFFCAQCGAFFGPEGFHEKDGKAYCRKDYFDMFA ${ }^{439}$ LIM3 $\mathrm{P}^{440}$ KCGGCARAILENYISALNTLWHPECFVCRECFTPFVNGSFFEHDGQPYCEVHYHERRG ${ }^{498}$ LIM4 $S^{499}$ LCSGCQKPITGRCITAMAKKFHPEHFVCAFCLKQLNKGTFKEQNDKPYCQNCFLKLFC 557
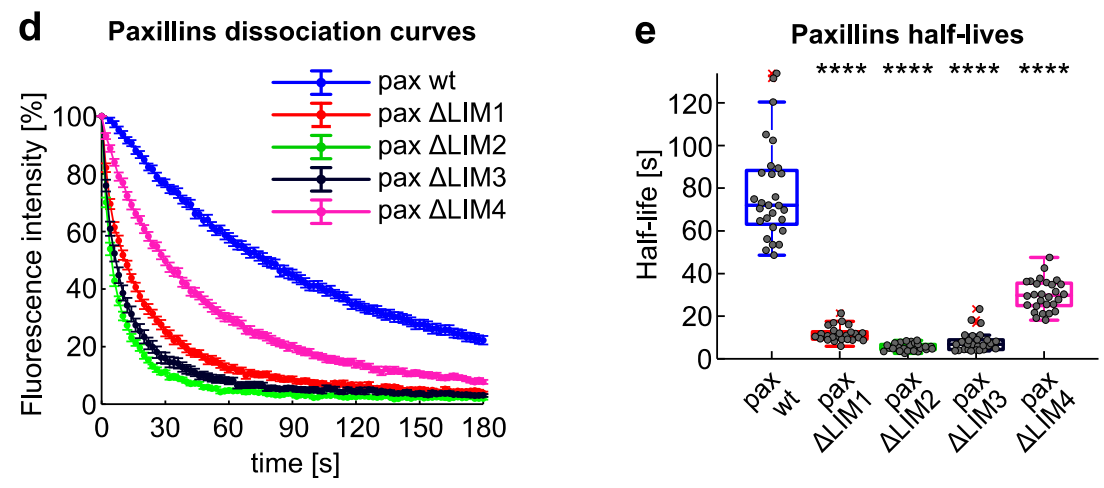

g

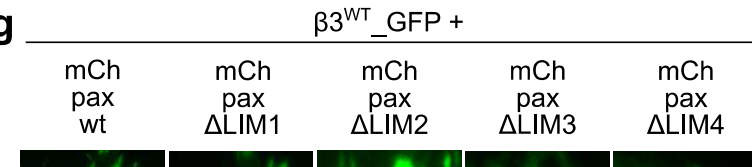
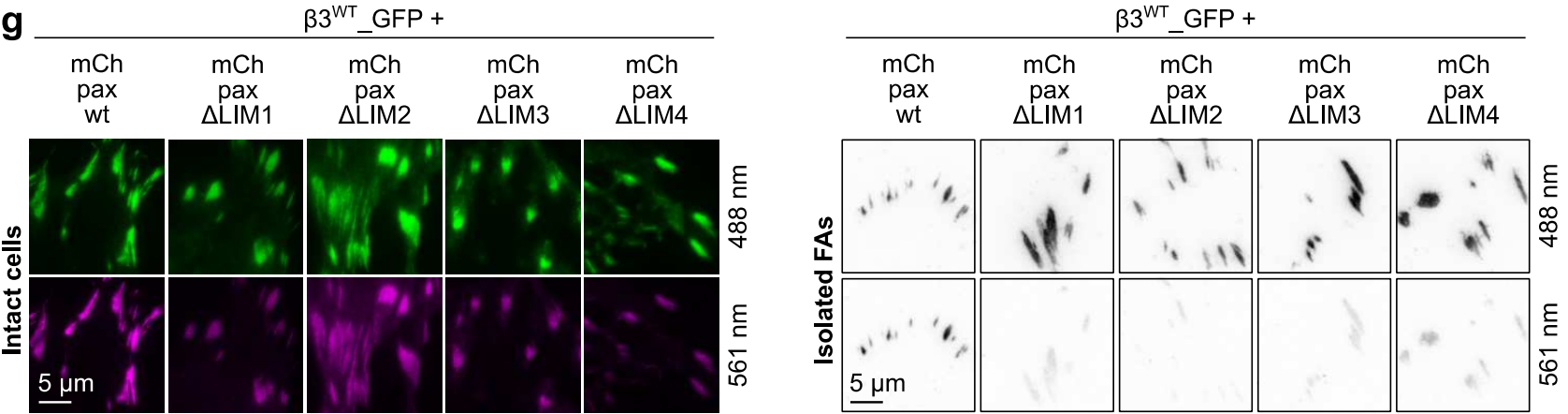

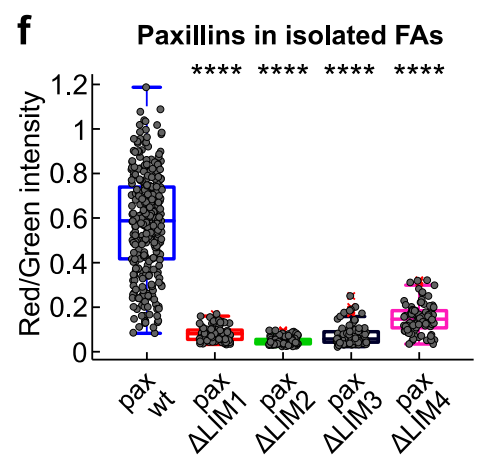

C

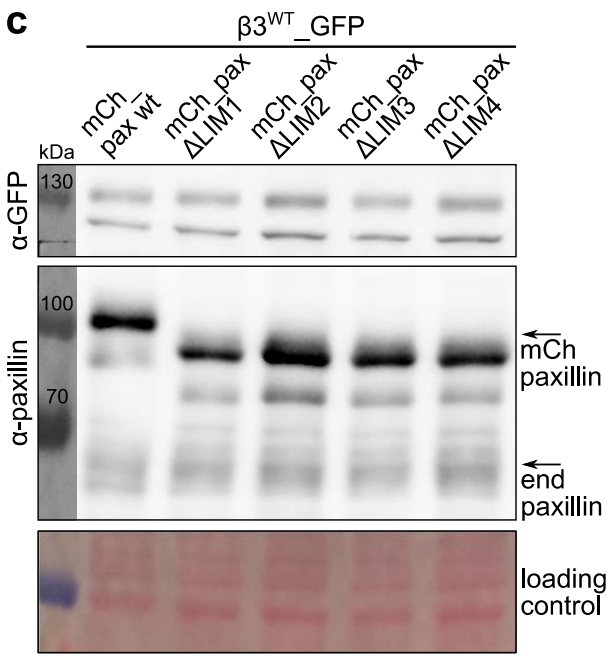

Figure 5 - LIM domain deletion analysis by photoactivation and FA-isolation. (a) Schematic representation of the paxillin LIM domain deletion mutants. All proteins were N-terminally tagged with PA-GFP or mCherry. (b) Alignment of the four paxillin LIM domains. Grey: zinc-binding residues. (c) Western blot of protein extracts from NIH-3T3 fibroblasts transiently co-expressing $\beta 3^{\mathrm{WT}}$ GFP and mCherry-tagged paxillin proteins, wt or deletion mutants. Loading control: Ponceau staining. (d) Experimental dissociation curves of PA-GFP_paxillin wt and deletion mutants from $\beta 3^{\mathrm{WT}}$ mCherry-positive FAs. (e) Box plot of the corresponding half-lives Statistical analysis is provided in Supplementary Table 3. (f) Quantification of mCherry_paxillin wt and mutants retained in FAs isolated from NIH-3T3 cells co-expressing $\beta 3^{\mathrm{WT}}$ GFP. Statistical analysis is provided in Supplementary Table 4. (g) Representative TIRF images of FAs in NIH-3T3 cells co-expressing $\beta 3^{\mathrm{WT}} \_$GFP and mCherry_paxillin proteins. Left panel: FAs in intact cells; right panel: isolated FAs. 
317 LIM domains are not interchangeable and LIM2 is the major determinant of paxillin 318 docking to FAs

319 Because of the confounding factor generated by the LIM domain deletion strategy, which abolished 320 the function of a LIM domain but at the same time also destroyed their respective spacing (with the 321 exception of LIM4 truncation), we developed the LIM domain replacement strategy (Fig. 6a and 322 Supplementary Fig. 4b).

323 A specific LIM1 domain-associated function was revealed by replacing it by either LIM2 or LIM3, 324 and assessing the FA-dissociation dynamics of the resulting paxillin 2234 and paxillin 3234 proteins 325 (Fig. 6b,c and Supplementary Fig. 2d and 3j). While both mutants partially rescued the deletion of 326 LIM1, none of them presented a level of FA-binding comparable to that of paxillin wt, indicating a 327 missing LIM1-depedent interaction. Nevertheless, LIM2 was slightly more efficient than LIM3 in 328 rescuing LIM1, proposing that despite the high degree of homology among their primary structure, 329 LIM2 presents some residues that better mimicked the precise and unique signature of LIM1.

330 The half-life of paxillin lacking LIM2 (paxillin $\Delta$ LIM2, $5.1 \mathrm{sec}$ ) was only slightly improved by 331 duplicating LIM1 (paxillin 1134, $22.1 \mathrm{sec}$ ) or LIM3 (paxillin 1334, $16.4 \mathrm{sec}$ ) (Fig. 6d,e and 332 Supplementary Fig. 2d and 3k). These data favor the hypothesis that the LIM2 domain does possess 333 exclusive features and an irreplaceable function that none of the other structurally similar paxillin 334 LIM domains can execute.

335 When based on the sole analysis of the LIM domain deletion mutants, we would have expected that 336 LIM3 is carrying key determinants of paxillin binding to FAs, confirming the previously proposed 337 findings by Brown et al. ${ }^{21}$. However, the LIM3 replacement by either LIM1 or LIM2, caused no 338 significant (paxillin 1214) and only a minor (paxillin 1224) acceleration of paxillin dissociation from 339 FAs, when compared to the wild-type protein (Fig. 6f,g and Supplementary Fig. 2d and 31). This 340 corresponds to an efficient rescuing of the LIM3 deletion and proposes that a LIM3 domain-specific 341 function is unlikely to be sequence-dependent, but rather relying on its overall 3D-folding, faithfully 342 reproduced by both LIM1 and LIM2. In fact, it raises the possibility that LIM3 has an important role 343 in creating the correct distance between LIM1-LIM2 and LIM4 domains, assuring their correct spatial 344 separation when binding to the FA-complex.

345 Finally, relying on a BiFC approach, we demonstrated that the paxillin binding to FAs, but not its 346 recruitment was altered by the LIM domain replacement strategy. Emblematic of these two distinct 347 phenomena is the analysis of paxillin 1334, which was among the least retained mutant (Fig. 6d,e), 348 showing a dissociation curve comparable to that of wild-type paxillin from $\beta 3^{\mathrm{VE} / \mathrm{YA}}$ integrin349 containing FAs (Fig. $2 \mathrm{~g}, \mathrm{~h}$ ), yet accomplishing a BiFC of $\beta 3^{\mathrm{WT}}$ integrin at least as efficient as the wt 
350 paxillin (Fig. 6h,i). Consistent BiFC results were obtained upon exchange of citrine fragments among 351 paxillin and integrin (Fig. 6j,k), confirming that the replacement of the LIM2 by LIM3 domain 352 resulted in a protein (paxillin 1334) unable to make stable interactions with FAs, which was however 353 well, but only transiently, recruited to these sites.

354 Overall, these data revealed that individual LIM domains have specific FA-interaction functions 355 linked to their respective position in the LIM domain repeats. Moreover, the efficient BiFC between 356 paxillin 1334 and the $\mathrm{C}$-terminus of $\beta 3$ integrin suggests that despite reduced retention in FAs and 357 the absence of the pivotal LIM2 domain, the mechanisms of recruitment of the LIMs-array in respect 358 to the C-terminal end of the integrin tail was not altered. 


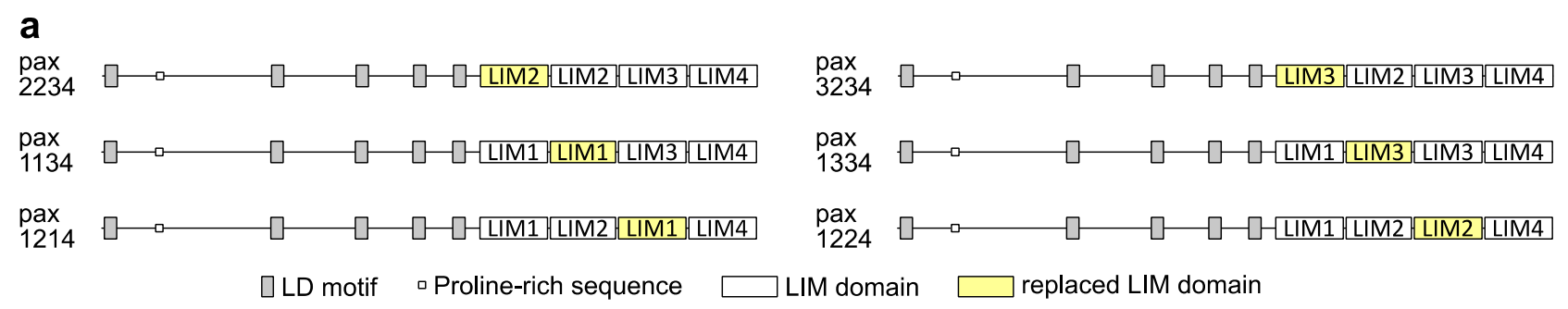

b Paxillins dissociation curves

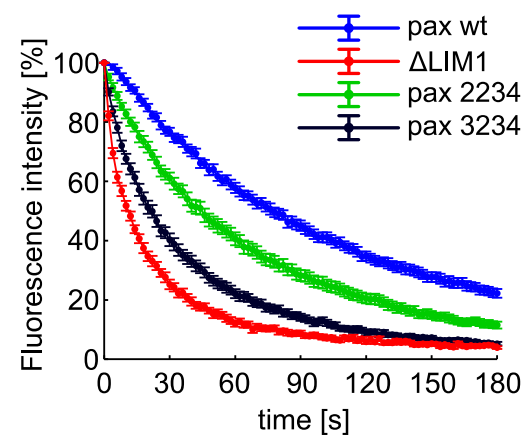

C Paxillins half-lives

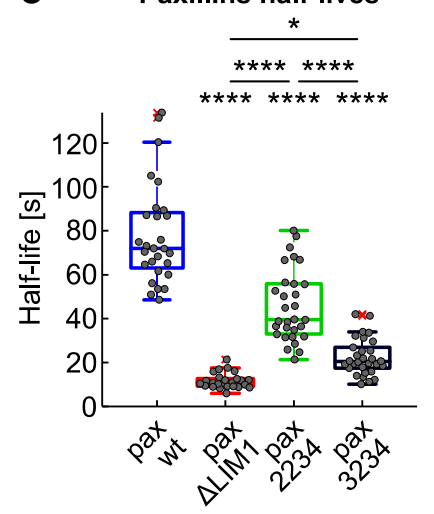

d

Paxillins dissociation curves

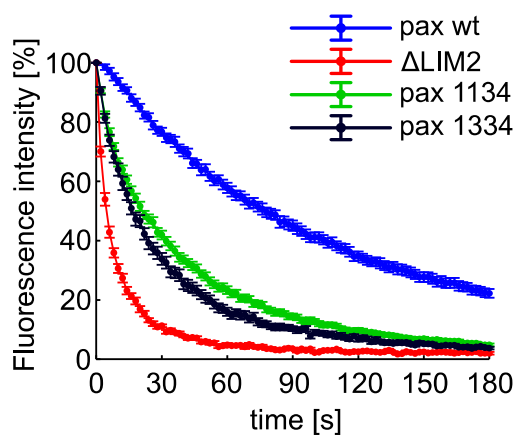

e

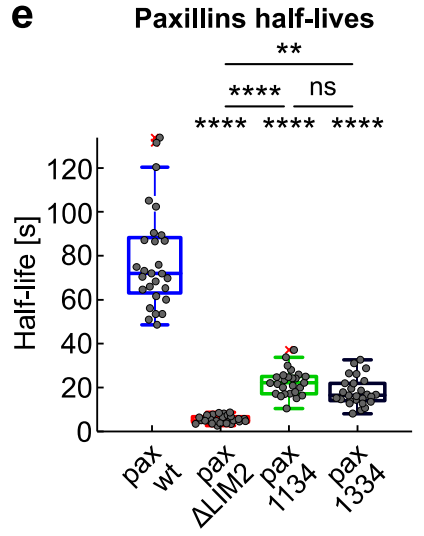

h

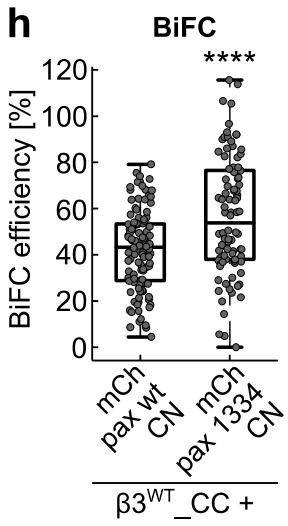

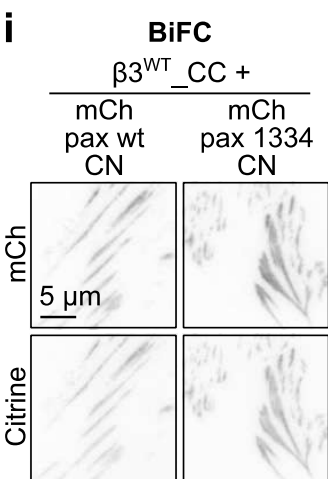

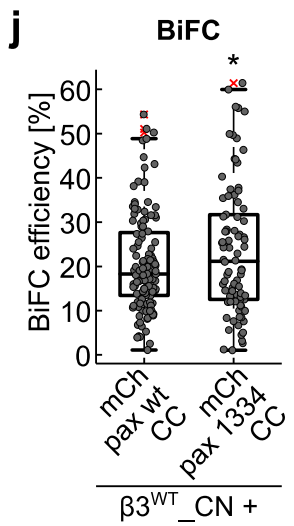

f

Paxillins dissociation curves

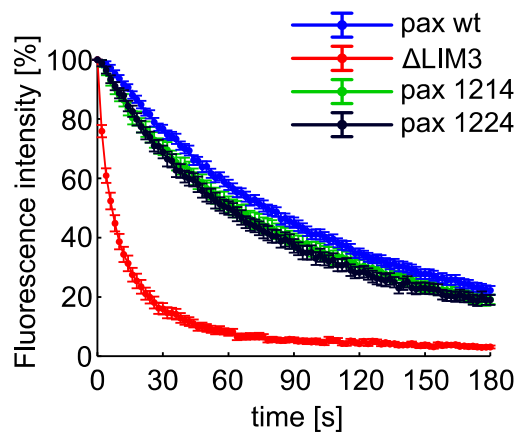

g Paxillins half-lives
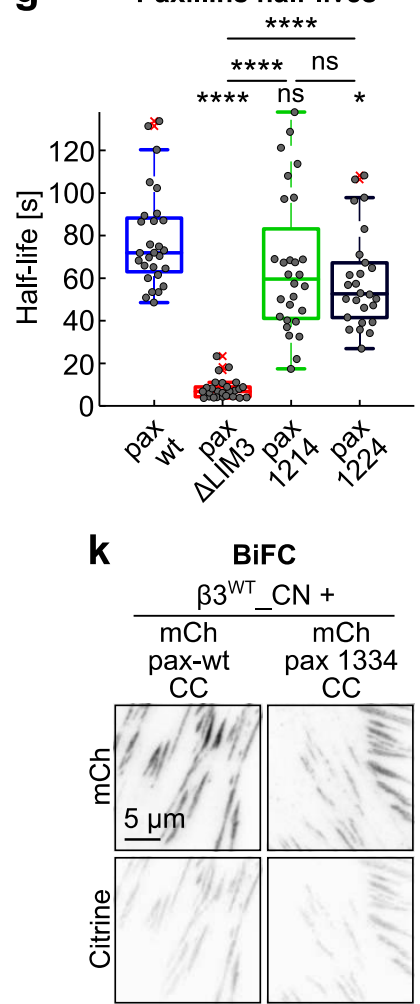

Figure 6 - LIM domain-specific functions assessed by the LIM domain replacement strategy. (a) Schematic representation of the paxillin mutants obtained by the LIM domain replacement strategy. From the top line: replacement of LIM1, replacement of LIM2 and replacement of LIM3. All proteins were N-terminally tagged with PA-GFP. (b,d,f) Experimental dissociation curves of PAGFP_paxillin wt and (b) LIM1 mutants, (d) LIM2 mutants and (f) LIM3 mutants, from $\beta 3$ mCherrypositive FAs. (c,e,g) Box plot of the half-lives of PA-GFP_paxillin wt and (c) LIM1 mutants, (e) LIM2 mutants and (g) LIM3 mutants in $\beta 3$ mCherry-positive FAs. Statistical analysis is provided in Supplementary Table 3. (h,i) BiFC upon co-expression of mCherry_paxillin_CN or its corresponding paxillin 1334 mutant and $\beta 3^{\mathrm{WT}}$ integrin $\mathrm{C}$-terminally tagged with the citrine $\mathrm{C}$-terminal fragment in NIH-3T3 cells. (h) Quantification of the BiFC efficiency. Satistical analysis is provided in 
Supplementary Table 1. (i) Representative TIRF images. (j,k) BiFC upon co-expression of 371 mCherry_paxillin_CC or its corresponding paxillin 1334 mutant and $\beta 3^{\text {WT }}$ integrin C-terminally 372 tagged with the citrine N-terminal fragment in NIH-3T3 cells. (j) Quantification of the BiFC 373 efficiency. Statistical analysis is provided in Supplementary Table 1. (k) Representative TIRF images.

\section{Paxillin LIM4 is a potential membrane binding and palmitoylated domain}

By sequence comparisons of the paxillin LIM domains, Beckerle and co-workers grouped the homologous LIM1, LIM2 and LIM3 but classified differently LIM4 ${ }^{22}$. When analyzed for specific features, LIM4 stands out for its remarkably high positive charge $(+5$ compared to $-1,-1$ and -2 of LIM1, LIM2 and LIM3 respectively). LIM4 is also unique for the presence of two conserved cysteines that do not coordinate the binding of zinc atoms $\left(\mathrm{Cys}^{512}\right.$ and $\left.\mathrm{Cys}^{557}-\mathrm{COOH}\right)$ and remain therefore potentially accessible for PTMs (Fig. 7a). Based on these peculiar and previously unnoticed features we investigated a possible membrane binding function for the paxillin LIM4 domain, intended to orient and stabilize paxillin in FAs. In fact, the cluster of basic residues could establish nonspecific electrostatic interactions with acidic phospholipids of the PM while the abovementioned cysteines can be $S$-acylated (i.e. palmitoylated), further strengthening this membrane association.

Considering the link between protein structure/function and selective pressure over evolution, an alignment of paxillin LIM4 domain across species was performed (Fig. 7a). It appeared that this domain is highly conserved, that the C-terminal cysteine, compared to $\mathrm{C}^{512}$, arose later during evolution and that much simpler organisms, although containing several sequence modifications, presented a perfectly intact GRC ${ }^{512}$ motif and an overall high net positive charge (Fig. 7a). As these well-preserved features may be pivotal for the functioning of the domain, we sought to determine their contribution to paxillin retention in FAs. Site-directed mutagenesis, preventing palmitoylation at $\mathrm{Cys}^{512 / 557}$ or abrogating LIM4 positive charge, did not affect paxillin expression levels (Fig. 7b). However, they reduced the half-life of paxillin in $\beta 3^{\mathrm{WT}}$ integrin-positive FAs, as shown by photoactivation experiments (Fig. 7c). Ensuring lipidation of the paxillin $\mathrm{C}^{512 / 557} \mathrm{~S}$ double mutant, by adding to the paxillin C-terminus a CaaX-box (PA-GFP_paxillinC ${ }^{512 / 557}$ S-CAIL), rescued paxillin FA-dissociation dynamics to wild-type levels (Fig. 7c). Interestingly, the mutation of the arginine within the conserved GRC motif $\left(\mathrm{R}^{511} \mathrm{Q}\right)$ was comparable to the $\mathrm{C}^{512} \mathrm{~S}$ mutation (Fig. $7 \mathrm{c}$ ), suggesting that this residue (and $\mathrm{G}^{510}$ as well) may function to allow the lipidation of the following cysteine. The neutralization of paxillin LIM4 positive charge, by means of the $\mathrm{RKK}^{511 / 532 / 554} \mathrm{QED}$ triple mutation, reduced the retention of paxillin in FAs to the same extent as the deletion of the entire LIM4 domain respect to the PM. 
403 The Acyl-Resin Assisted Capture assay (Acyl-RAC), a valid tool to detect palmitoylated proteins 404 among a total cell lysate, revealed a small but constant fraction of paxillin in the pool of palmitoylated 405 proteins (Fig. 7d). The amount of palmitoylated paxillin strongly increased when, by means of the 406 previously exploited CaaX-box (mCherry_paxilin-CAIL), an irreversible geranylgeranyl lipid anchor 407 was coupled to the its carboxyl-terminus. Only in this context we appreciated the effect of the 408 treatment with 2-bromopalmitate (2BP) that, competing with endogenous palmitate, acts as a general 409 inhibitor of palmitate incorporation. Caveolin-1, because of its essentially irreversible palmitoylation $410{ }^{60}$ is not sensitive to $2 \mathrm{BP}$ treatment and was therefore used as an internal positive control.

411 Overall, these data highlighted the relevance of LIM4 positive charges and demonstrated the 412 palmitoylation of the paxillin protein, which is in line with a possible membrane binding function of 413 the LIM4 domain.

\section{a Conservation of the paxillin LIM4 domain across species}

\begin{tabular}{|c|c|c|c|}
\hline & & & \\
\hline H. sapiens & PXN & CSGCQKPITGRCITAMAKKFHPEHFVCAFCLKQLNKGTFKEQNDKPYCQNCFLKLFC & +5 \\
\hline R. norvegicus & PXN & CSGCQKPITGRCITAMAKKFHPEHFVCAFCLKQLNKGTFKEQNDKPYCQSCFLKLFC & +5 \\
\hline M. musculus & PXN & CSGCQKPITGRCITAMAKKFHPEHFVCAFCLKQLNKGTFKEQNDKPYCQSCFVKLFC & +5 \\
\hline G. gallus & PXN & CSGCQKPITGRCITAMGKKFHPEHFVCAFCLKQLNKGTFKEQNDKPYCQNCFLKLFC & +5 \\
\hline X. laevis & PXN & CSGCQKPITGRCITAMGKKFHPEHFVCAFCLKQLNKGTFKEQNDKPYCQNCFVKLFC & +5 \\
\hline D. rerio & PXNA & CSGCQKPITGRCITAMGKKFHPEHFVCAFCLKQLNKGTFKEQNDKPYCQSCFVKLFS & +5 \\
\hline D. melanogaster & Pax & CAGCSKPITGRCITAMFKKFHPEHFVCAFCLKQLNKGTFKEQKDKPYCHTCFDKIFG & +5 \\
\hline C. elegans & PXL1 & CSQCRGAINGRCVAAMGRKFHPEHFRCSYCNHQLTKGTEKEVDRRPFCHKCYNNTYALTPA & +6 \\
\hline D. discoideum & PAXB & CSGCGKAVSGRCVDALDKKWHPEHFVCAFCMNPLAGGSYTANNGKPYCKGCHNKLFA & +3 \\
\hline LIM4 t & & FCLEQLNKGTFKEQNDKPYCQNCFLDLFC & 0 \\
\hline
\end{tabular}

b
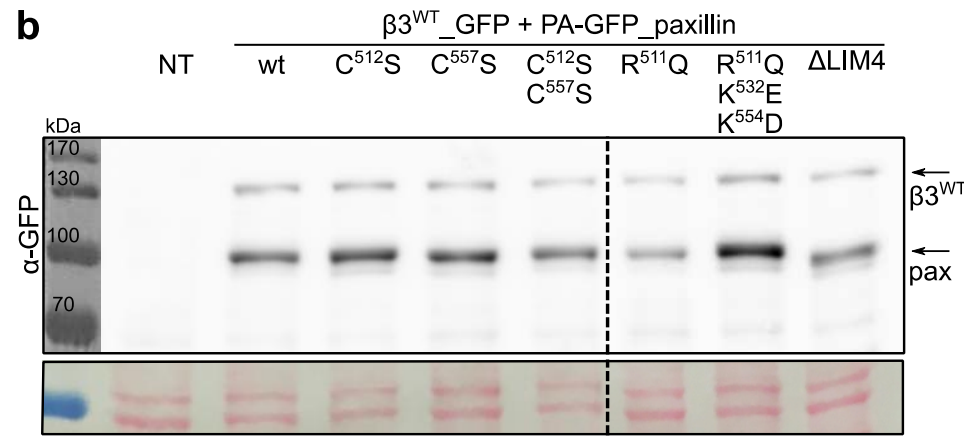

d

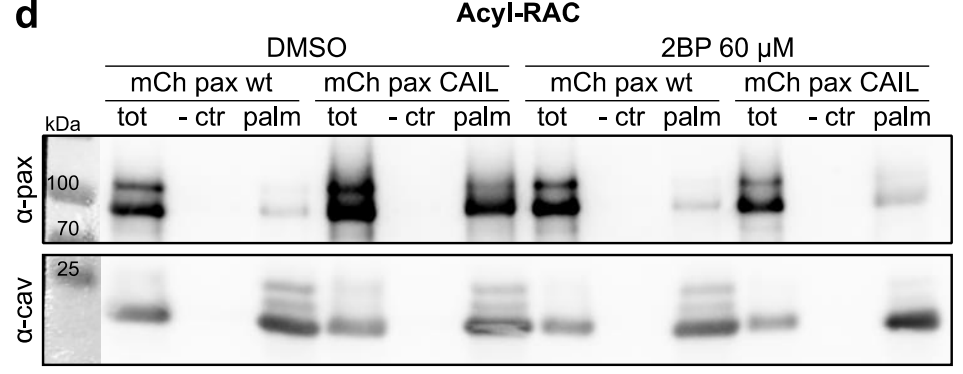

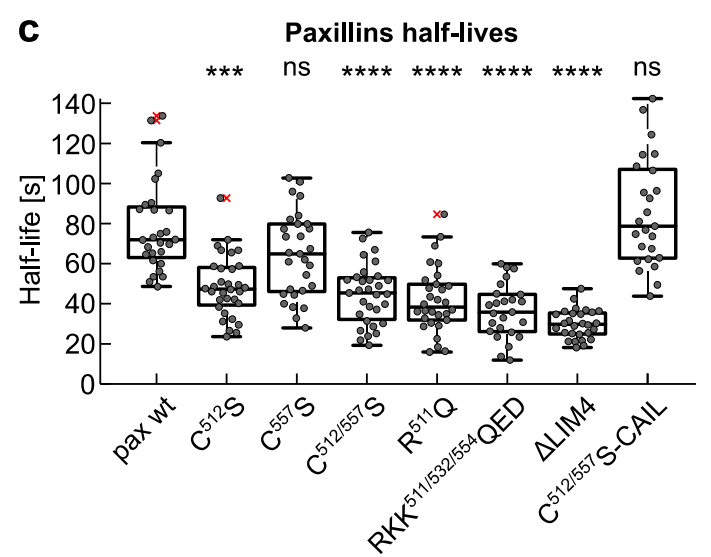

415 Figure 7 - Paxillin LIM4 domain carries membrane binding features relevant for FA binding. 416 (a) Alignment of the amino acid sequence of paxillin LIM4 across species and triple human paxillin 417 LIM4 mutant (bottom). The latter was obtained by replacing three basic amino acids with neutral or 
acidic residues found at the corresponding positions in other paxillin LIM domains 419 (RKK $\left.{ }^{511 / 532 / 554} \mathrm{QED}\right)$. Grey: zinc-binding residues; blue: positive residues; red: negative residues; bold: non-conserved residues in respect to the human paxillin LIM4 domain; arrows indicate the conserved free cysteines; yellow: conserved GRC motif. (b) Expression analysis, via western blot, of the paxillin LIM4 mutants. Loading control: Ponceau staining. (c) Box plot of the half-lives of PAGFP_paxillin wt and LIM4 mutants in $\beta 3^{\mathrm{WT}}$ _mCherry-positive FAs. Statistical analysis is provided in Supplementary Table 3. (d) Acyl-Resin Assisted Capture assay from cell transiently transfected with mCherry_paxillin wt or mCherry_paxillin-CAIL, untreated (DMSO) or treated with 2bromopalmitate. NT: not transfected; tot: total cell lysate; - ctr: negative control (tris-base); palm: fraction of palmitoylated proteins (hydroxylamine); 2BP: 2-bromopalmitate.

\section{BiFC shows a defined spatial orientation of paxillin LIM domains within focal adhesions}

A possible function of a membrane-binding activity of LIM4, is to specifically orient the entire LIM domain-array within FAs. Previous iPALM studies revealed a close membrane association of both paxillin termini ${ }^{61}$. However, based on various structures of two tandemly-organized LIM domains (e.g. PDB ID: 1RUT and PDB ID: 2DFY), it is conceivable that the paxillin LIM1-LIM2-LIM3LIM4 array has an extended conformation. Because the BiFC efficiency is imposed by the relative position of the proteins under analysis, as well as by the spatial orientation of the complementary fluorophore fragments, we attempted to reveal the orientation of paxillin in respect to the PM by BiFC. Accordingly, as summarized in Fig. 8a, differentially tagged recombinant paxillins were tested for their ability to complement, in the context of $\beta 3$-expressing fibroblasts (Swiss-3T3 ${ }^{43}$ ), a membrane-anchored citrine fragment so localized via the PM-targeting peptide of Lck ${ }^{62}$. Despite the flexible nature of the paxillin amino-terminal half ${ }^{63}$, only the paxillin C-terminus appeared close enough to the lipid bilayer for efficient BiFC with the PM-residing complementary probe (Fig. 8b,c). Importantly, the spatial orientation of paxillin was encoded by the LIM domains, which yielded comparable results in the absence of the paxillin N-terminus (Fig. 8b,c). The swapping of citrine fragments between the PM probe and paxillin/LIMs (Supplementary Fig. 1c) produced analogous outcomes, confirming the preferred complementation of the PM-localized CN fragment by Cterminally over N-terminally CC-tagged paxillin/LIMs proteins (Supplementary Fig. 1d). Interestingly, despite the general distribution of the PM-anchored citrine fragments within the lipid bilayer (Supplementary Fig. 1f), the BiFC-driven paxillin-PM association identified in virtue of the citrine fluorescence, did not affect the specific FA-localization of paxillin (Fig. 8c). On the other hand, in the few cells that showed an interaction between the N-terminus of LIMs and the PM probe, 451 that when trapped in an "inverted" orientation, LIM domains have a low affinity for FAs 452 (Supplementary Fig. 1h). 
453 To gather further insights about the mechanism of paxillin binding to the FA-complex, we mapped 454 the relative position of the paxillin extremities compared to the $\beta 3$ integrin tail, applying a similar 455 BiFC strategy (Fig. 8a). Efficient complementation of CC-tagged $\beta 3^{\mathrm{WT}}$ integrin was obtained only 456 when the matching citrine fragment was fused to the C-terminus of paxillin (Fig. 8d,e). Similarly, the 457 BiFC efficiencies of the LIMs-only constructs were comparable to that of the corresponding full458 length protein (Fig. 8d,e), and insensitive to the swapping of the complementary citrine fragments 459 (Supplementary Fig. 1c), demonstrating the significantly higher interaction of the paxillin C-terminus 460 with the integrin cytoplasmic tail (Supplementary Fig. 1d).

461 Although the sliding of $\beta 3^{\mathrm{VE} / \mathrm{YA}}$ adhesions was rescued by directly joining paxillin and the integrin 462 receptor via BiFC (Fig. 1i and Supplementary Fig. 1b), it is still controversial whether paxillin 463 mediates FA stabilization by direct $\beta$ integrin binding ${ }^{21,49,50}$. We therefore asked whether an integrin464 independent binding of paxillin could also rescue the sliding of $\beta 3^{\mathrm{VE} / \mathrm{YA}}$ adhesions. Leveraging the 465 newly disclosed knowledge about the structural organization of adhesion complexes, an alternative, 466 LIM4-mediated paxillin-FA interaction was stabilized, in order to evaluate its potential to rescue FA467 sliding. The irreversible anchoring of LIM4 to the PM was achieved by using the SpyTag/SpyCatcher 468 technology ${ }^{64}$ as presented in Fig. 8f. Upon co-expression of mCherry_paxillin_SpyTag and 469 PM_SpyCatcher a spontaneous reaction results in a stable isopeptide bond, which covalently 470 conjugated the two recombinant proteins (Fig. 8f). By this method, paxillin was depleted from the 471 cytoplasm, in favor of an enhanced PM localization (Supplementary Fig. 1i), which induced a slower 472 recovery in FAs after photobleaching (FRAP) (Supplementary Fig. 1j,k). The longer half-life and the 473 increased immobile fraction of such PM-anchored paxillin in FAs, effectively demonstrated the 474 occurrence of the cross-linking reaction. The latter, likely prolonging paxillin residency time within 475 FAs, rescued the $\beta 3^{\mathrm{VE} / \mathrm{YA}}$ integrin instability (Fig. 8g) and suggested that paxillin stability in adhesions 476 is a key factor in activating the clutch between the substrate-bound integrin and the retrogradely 477 moving F-actin network. Interestingly, strengthening paxillin binding to the PM compensate for the 478 loss of paxillin binding to $\beta 3$ integrin $\left(\beta 3^{\mathrm{VE} / \mathrm{YA}}\right)$. 

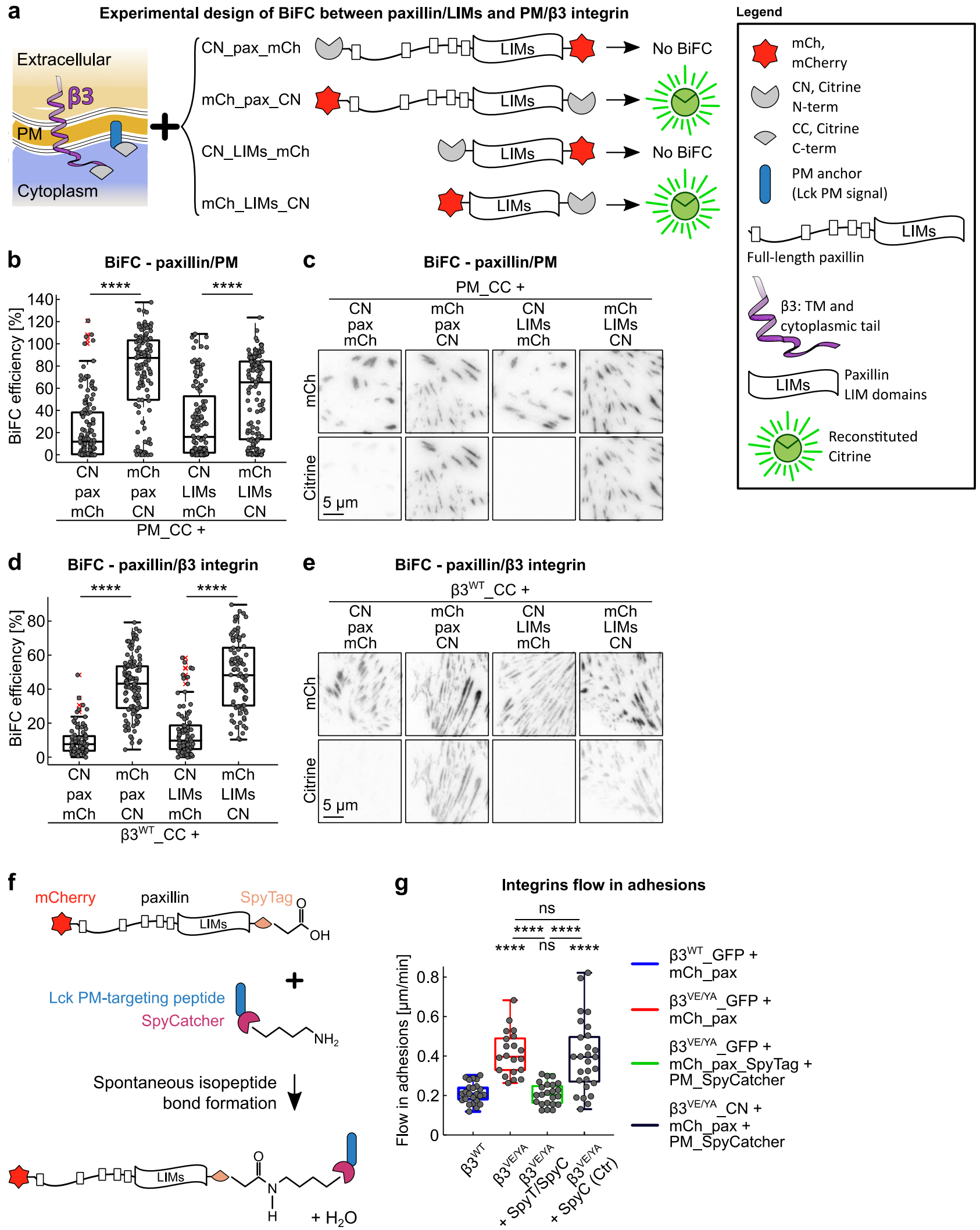

Figure 8 - Preferential orientation of the paxillin LIM domains within FAs. (a) Overview of the molecules tested with the $\mathrm{BiFC}$ assays and a summary outcome, in terms of citrine fluorescence, of their pairwise expression. (b,d) Quantification of the BiFC signal generated by the co-expression of each of the paxillin constructs shown in figure a with (b) the plasma membrane-localized CC fragment, in Swiss-3T3 or with (d) the $\beta 3^{\mathrm{WT}}$ C-terminally tagged with $\mathrm{CC}$, in NIH-3T3 cells. Statistical analysis is provided in Supplementary Table 1. (c,e) Representative TIRF images of 
486 experiments quantified in b and d. (f) Schematic representation of the functioning 487 SpyTag/SpyCatcher technology ${ }^{64}$ and of how it has been used in our cellular system. (g) 488 Quantification of the integrins flow in adhesions, expressed as mean displacement over time $489(\mu \mathrm{m} / \mathrm{min})$ per replicate. Statistical analysis is provided in Supplementary Table 2. 


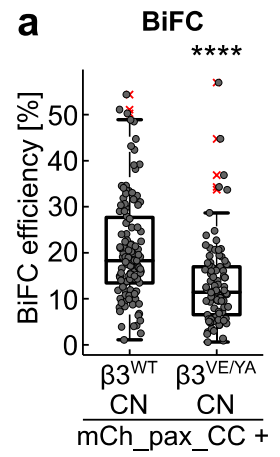

b

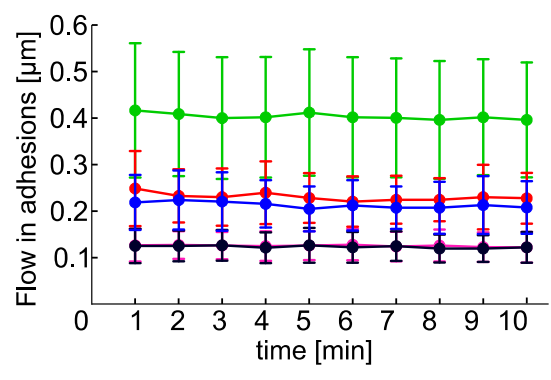

工 ${ }^{\beta 3^{\mathrm{WT}} \text { GFP + }}$

mCh_pax

$\mp \beta 3^{\mathrm{VE}} \mathrm{GFP}+$

工 $\beta 3^{\mathrm{VENA}}$ GFP +

I $\mathrm{mCh} \_$pax

I- $\beta 3^{\mathrm{VE} / \mathrm{P} A} \mathrm{CN}+$

ICh_pax_CC
$\mp$ I $3^{\text {WT }} \mathrm{CN}+$
$\mathrm{mCh}$-pax_CC
C

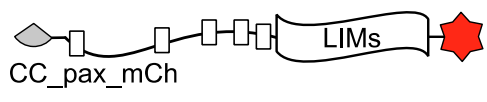
CC_pax_mCh

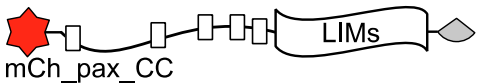

CC_LIMs_mCh $O$ LIMs

mCh_LIMs_cc L LIMs

Legend

$\mathrm{mCh}, \mathrm{mCherry} \circlearrowleft \mathrm{cc}$, Citrine C-term
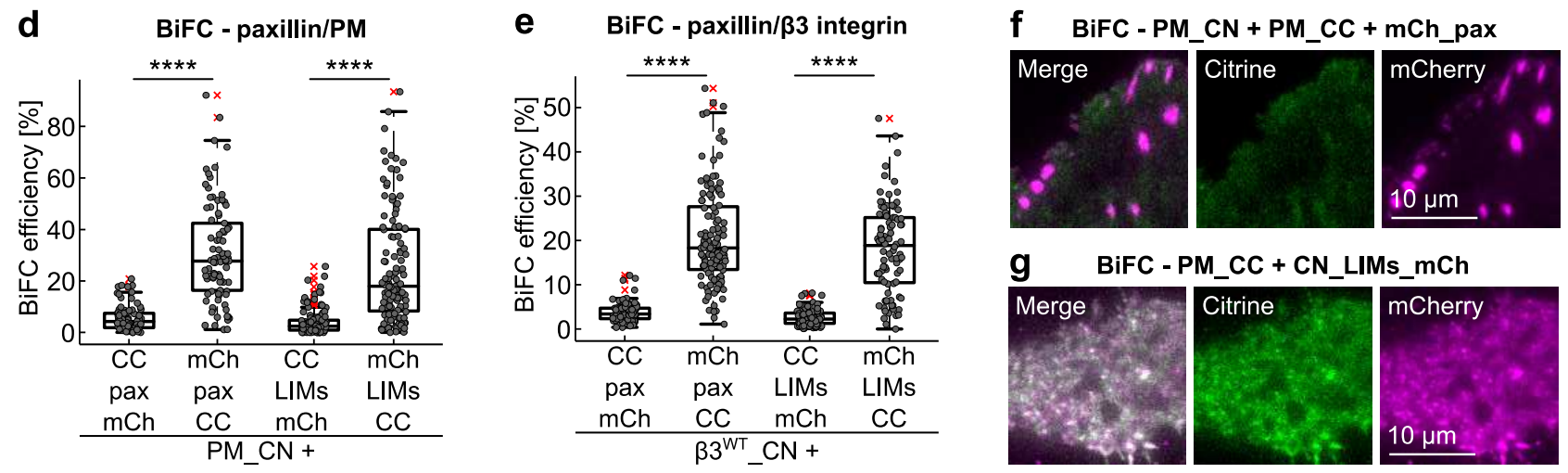

h
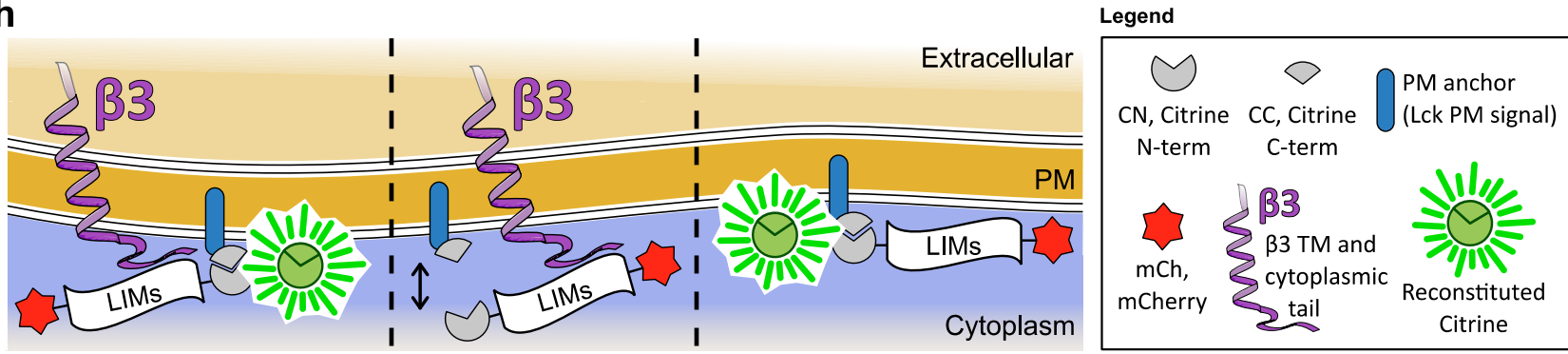

i

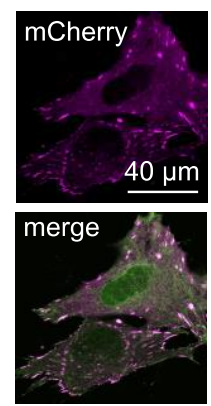

$\beta 3^{W T}$ GFP

mCh_pax

PM_SpyCatcher j

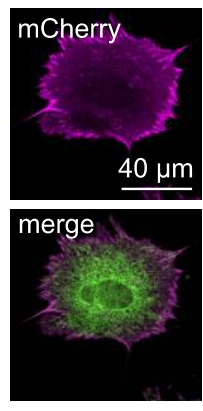

$\beta 3^{W T}$ GFP

mCh_pax_SpyTag

mM_SpyCatcher
FRAP - Recovery curves

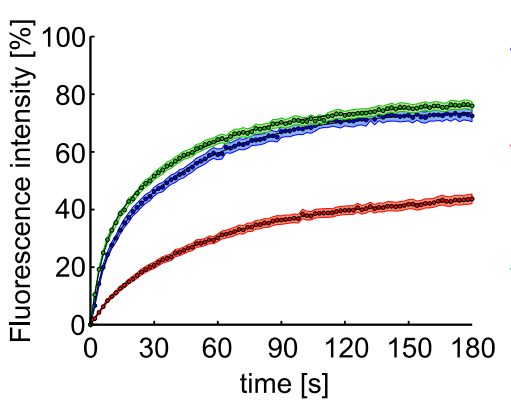

$-\beta 3^{\mathrm{WT}}{ }_{\text {GFP }}+$

mCh_pax

- $\beta 3^{\text {WT }}$ GFP + mCh_pax_SpyTag PM_SpyCatcher

$-\begin{aligned} & \beta 3^{\mathrm{WT}} \text { GFP }+ \\ & \mathrm{mCh} \text { pax }+\end{aligned}$ PM SpyCatcher

\section{K Paxillin half-life}

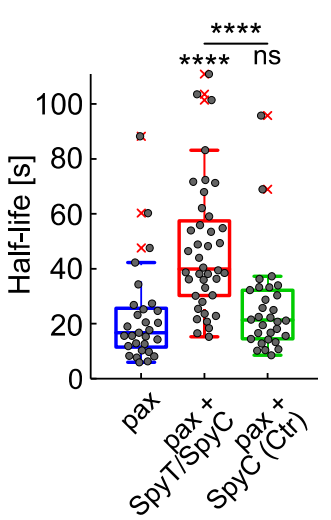

Supplementary Figure 1 - BiFC and SpyTag/SpyCatcher assays to study focal adhesions organization and function. (a) Quantification of the BiFC efficiency upon co-expression of mCherry_paxillin_CC and $\beta 3^{\mathrm{WT}}$ CN or $\beta 3^{\mathrm{VE} / \mathrm{YA}}{ }_{-} \mathrm{CN}$ in NIH-3T3 cells. Statistical analysis is provided in Supplementary Table 1. (b) Quantification of the $\beta 3$ integrins flow in adhesions, expressed as displacement over time. Error bar \pm SD. Statistical analysis is provided in Supplementary Table 2. (c) 
in BiFC assays with alternatively PM_CN or $\beta 3^{\mathrm{WT}}{ }_{-} \mathrm{CN}$. (d,e) Quantification of the BiFC signal generated by the co-expression of each of the paxillin constructs shown in figure $\mathbf{c}$ with (d) the plasma membrane-localized $\mathrm{CN}$ fragment, in Swiss-3T3 or with (e) the $\beta 3^{\mathrm{WT}}$ integrin C-terminally tagged with CN, in NIH-3T3 cells. Statistical analysis is provided in Supplementary Table 1. (f) Representative TIRF images of a triple positive Swiss-3T3 fibroblast, co-expressing mCherry_paxillin and the two complementary citrine fragments, each one fused to the PM-targeting peptide. While the BiFC signal is localized throughout to the plasma membrane, mCherry_paxillin only appears in the discreates spots of FAs. Brightness and contrast automatically optimized. (g) Example of a Swiss-3T3 fibroblast in which the co-expression of CN_LIMs_mCherry and PM_CC led to substantial BiFC and concomitant mis-localization of LIMs. Brightness and contrast automatically optimized. (h) Schematic representation of the possible scenarios, in terms of BiFC generation, upon co-expression of $\mathrm{CN}$-tagged LIMs recombinant proteins and the PM-localized complementary citrine fragment. Left: the $\mathrm{CN}$ fragment at the C-terminus of LIMs leads to BiFC in adhesions. Middle: the $\mathrm{CN}$ fragment in front of LIMs is not compatible with BiFC in adhesions. Right: the CN fragment in front of LIMs can complement PM-localized CC outside adhesions. (i) Differential paxillin distribution among FAs, cytosol and PM, in the absence (left) and in the presence (right) of the irreversible fusion of the C-terminus to the PM_SpyCatcher. (j) Florescence Recovery after Photobleaching (FRAP) of paxillin localized to $\beta 3^{\text {WT }}$ _GFP-positive FAs, in the absence (mCherry_paxillin and mCherry_paxillin + PM_SpyCatcher, control) and in the presence (mCherry_paxillin_SpyTag + PM_SpyCatcher) of the irreversible fusion of the C-terminus to the PM_SpyCatcher. (k) Box plot of the half-lives of paxillin in $33^{\mathrm{WT}}$ _GFP-positive FAs. Statistical analysis is provided in Supplementary Table 3. 

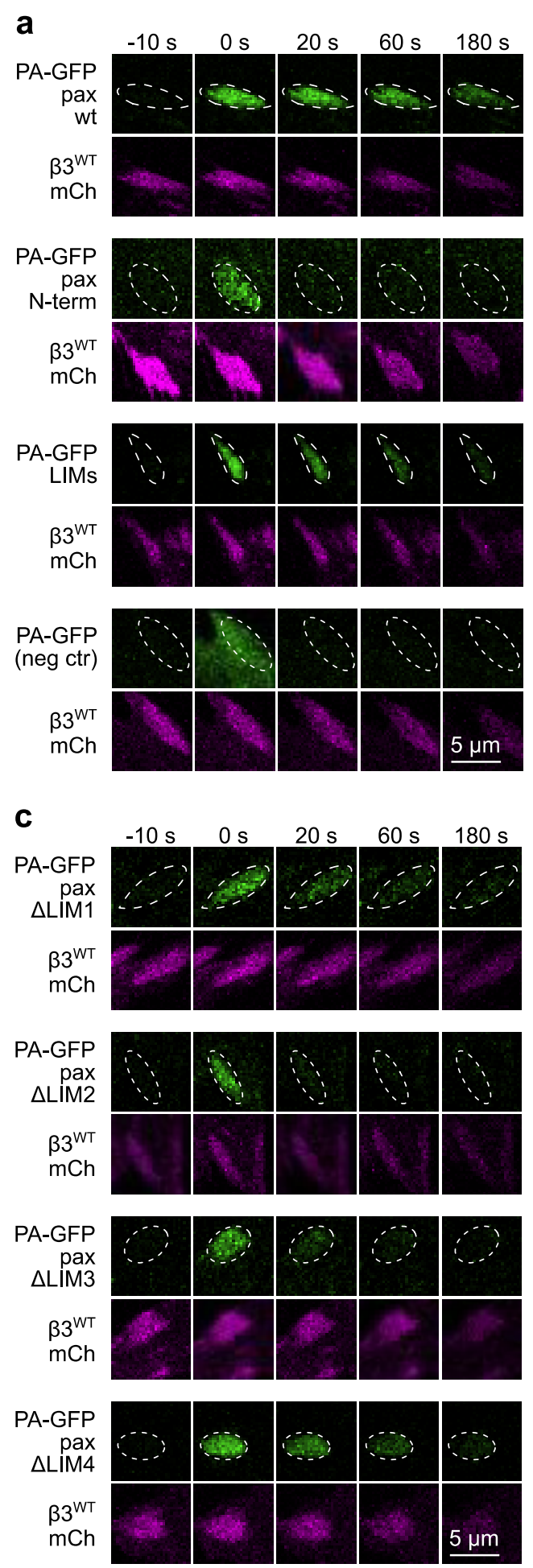

b

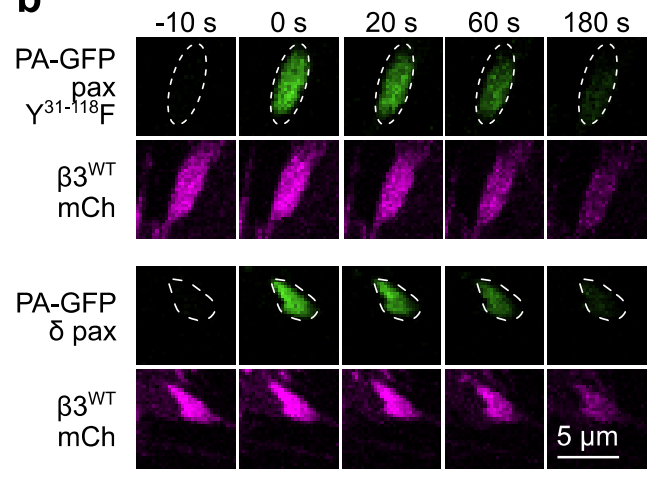

d
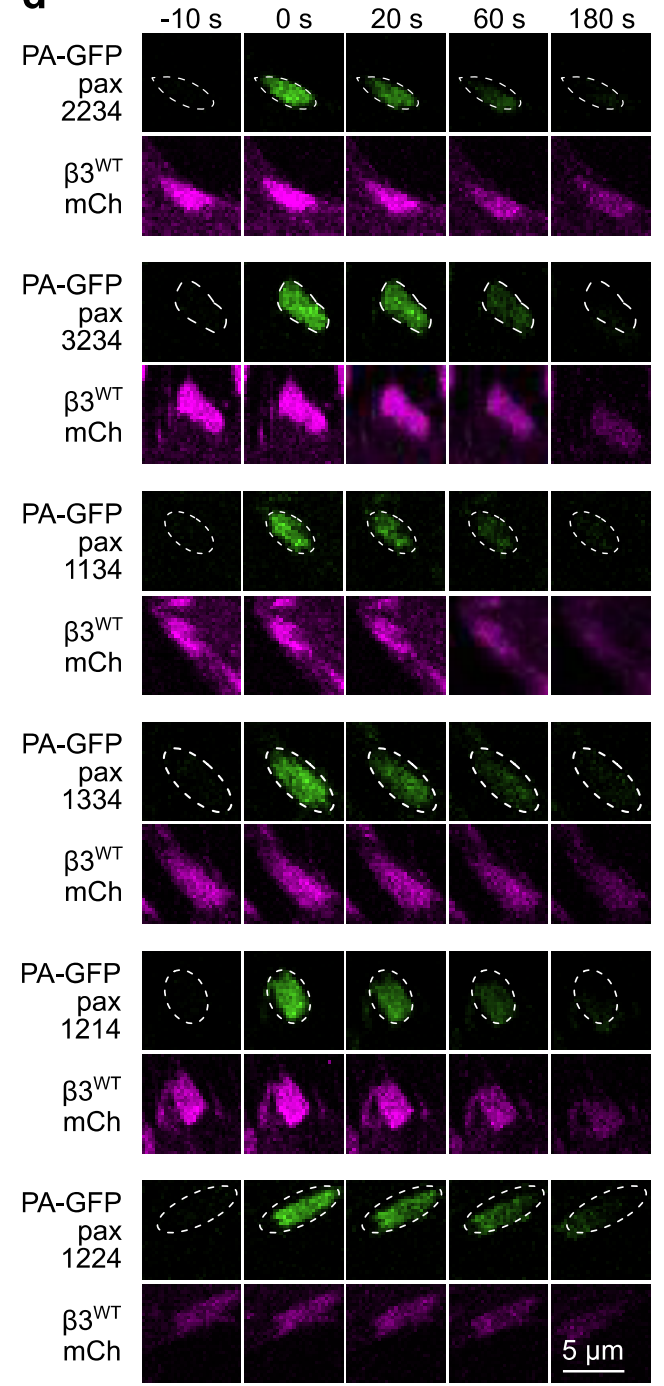

521 Supplementary Figure 2 - Photoactivation time series of paxillin in $\boldsymbol{\beta 3}^{\mathrm{WT}}$ _mCherry-positive 522 adhesions. (a-d) Images of photoactivation time series of paxillin wt and mutants, transiently co523 expressed with $\beta 3^{\mathrm{WT}}$ _mCherry integrin in NIH-3T3 cells. Brightness and contrast of images in the 524 green channel were adjusted to the image at to. PA-GFP: photoactivatable GFP; mCh: mCherry. 

- experimental -

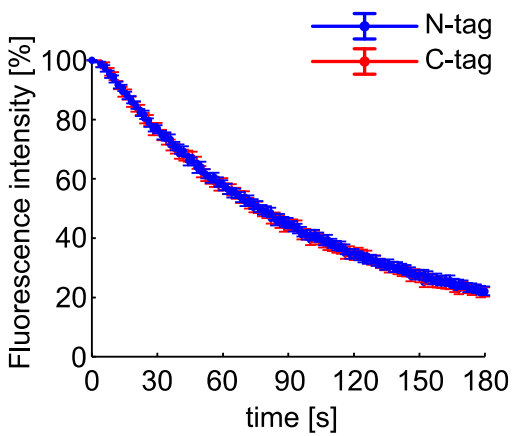

d $\quad$ B3 integrins dissociation curves double decaying exponentials model

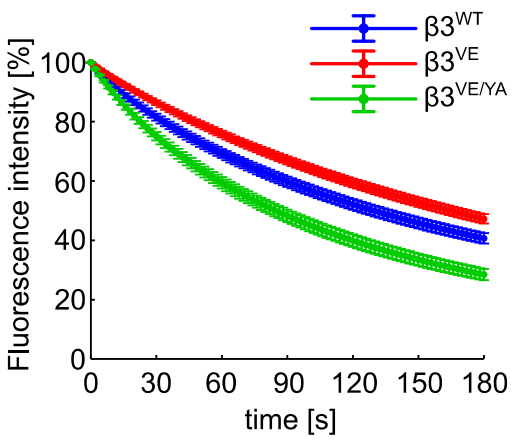

g Paxillins dissociation curves double decaying exponentials model

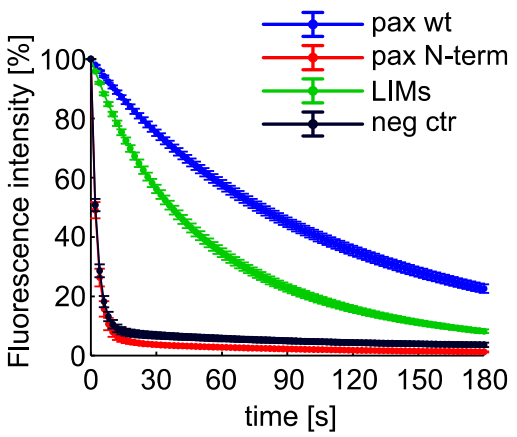

j Paxillins dissociation curves double decaying exponentials model

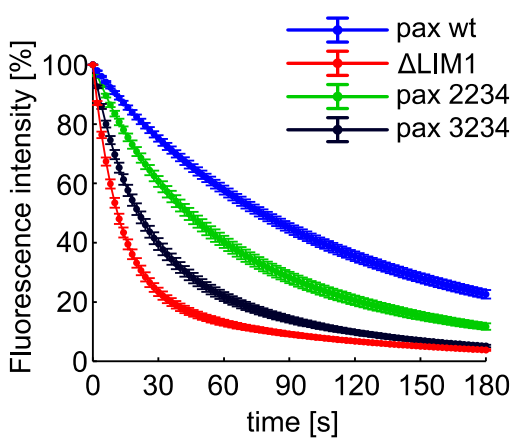

b Paxillins dissociation curves double decaying exponentials model

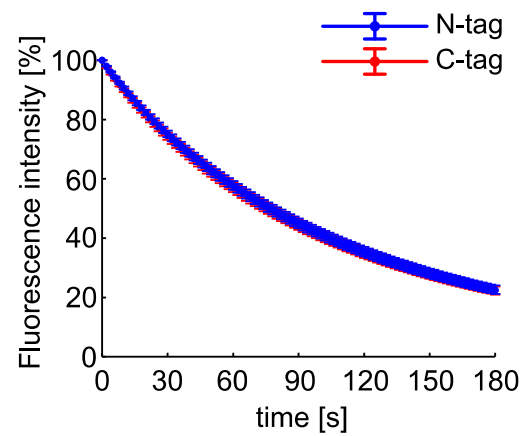

e

Talin dissociation curves double decaying exponentials model

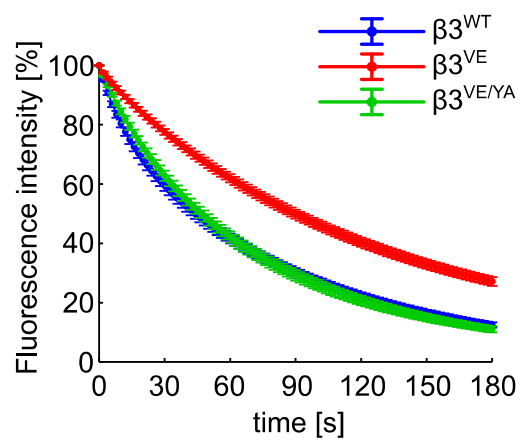

h Paxillins dissociation curves double decaying exponentials model

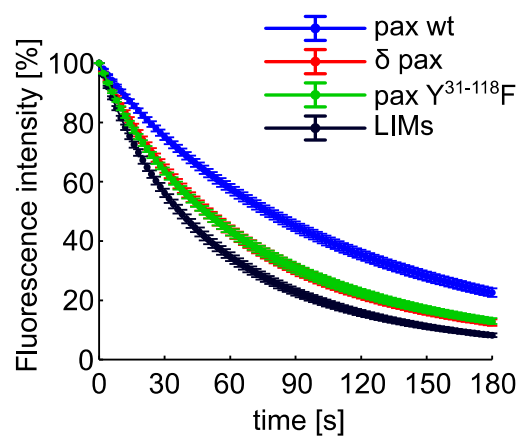

k Paxillins dissociation curves double decaying exponentials model

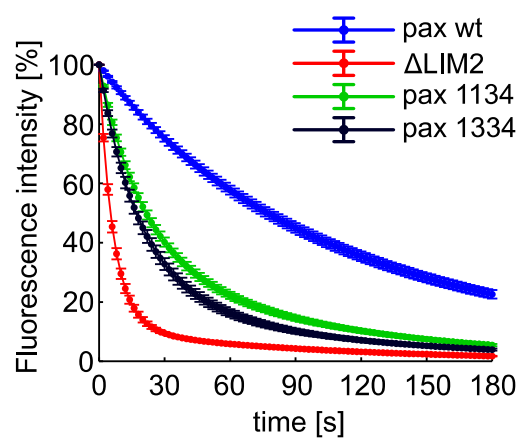

C

Paxillins

half-lives

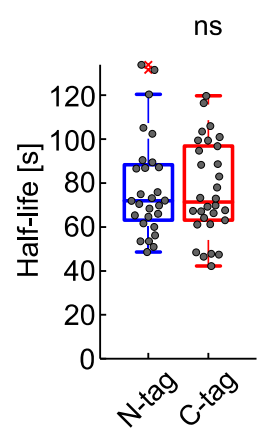

f

Paxillin dissociation curves double decaying exponentials model

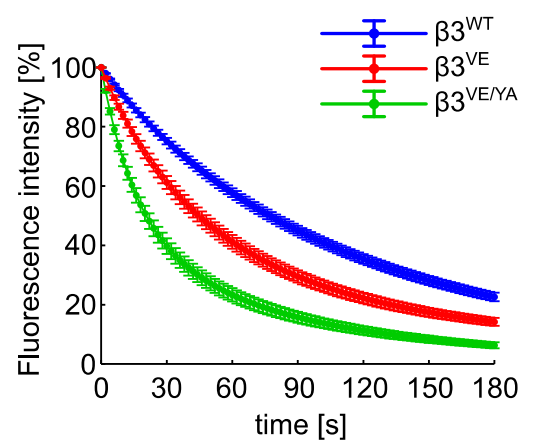

i

Paxillins dissociation curves double decaying exponentials model

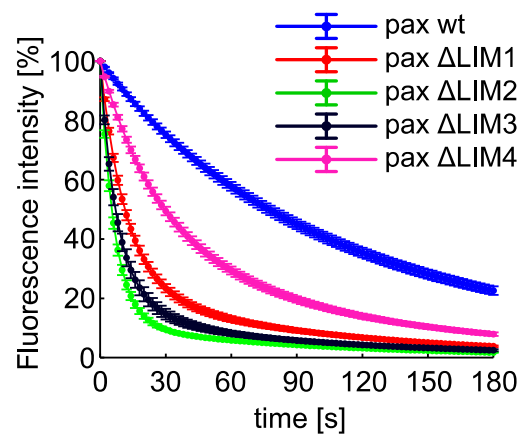

Paxillins dissociation curves double decaying exponentials model

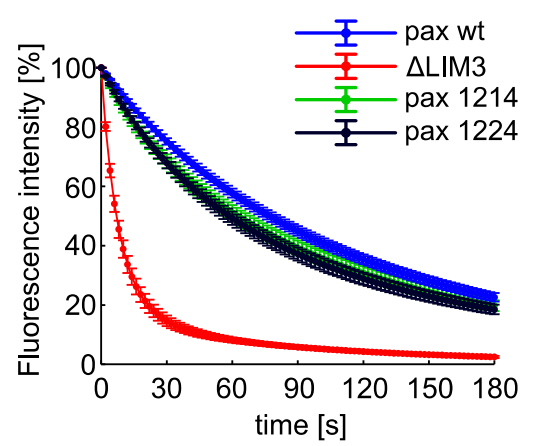
protein dissociation rates. (a) Experimental dissociation curves of PA-GFP_paxillin (N-tag) and paxillin_PA-GFP (C-tag) from $\beta 3$ mCherry-positive FAs. (c) Box plot of the half-lives of PA- 
GFP_paxillin (N-tag) and paxillin_PA-GFP (C-tag) in $\beta 3$ mCherry-positive FAs. Statistical analysis is provided in Supplementary Table 3. (b,f-I) Theoretical model of dissociation of PA-GFP-tagged paxillin proteins from $\beta 3$ mCherry-positive FAs. (d,e) Theoretical model of dissociation of (d) B3_PA-GFP integrins from mCherry_paxillin-positive FAs and of (e) PA-GFP_talin from B3_mCherry-positive FAs.

a paxillin $\triangle \mathrm{LIM} 1$

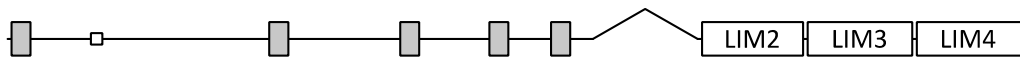
TVAKI ${ }^{322}$ VCGACKKPIAGQVVTAMGKTWHPEHFVCTHCQEEIGSRNFFERDGQPYCEKDYHNLFS ${ }^{380}{ }_{1}$ IRCY

paxillin $\Delta \mathrm{LIM} 2$

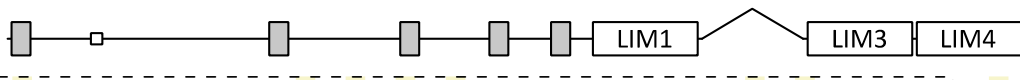

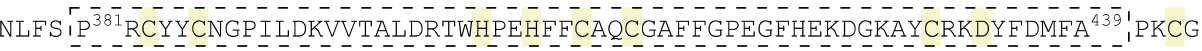

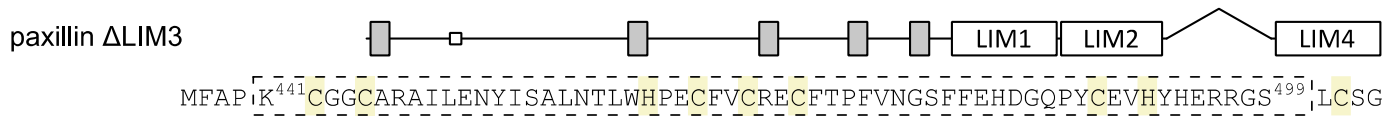

paxillin $\triangle$ LIM4 RRGSIII ${ }^{500}$ CSGCQKPITGRCITAMAKKFHPEHFVCAFCLKQLNKGTEKEQNDKPYCQNCFLKLFC ${ }^{557}-\mathrm{COOH}_{1}$

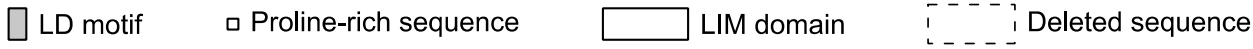
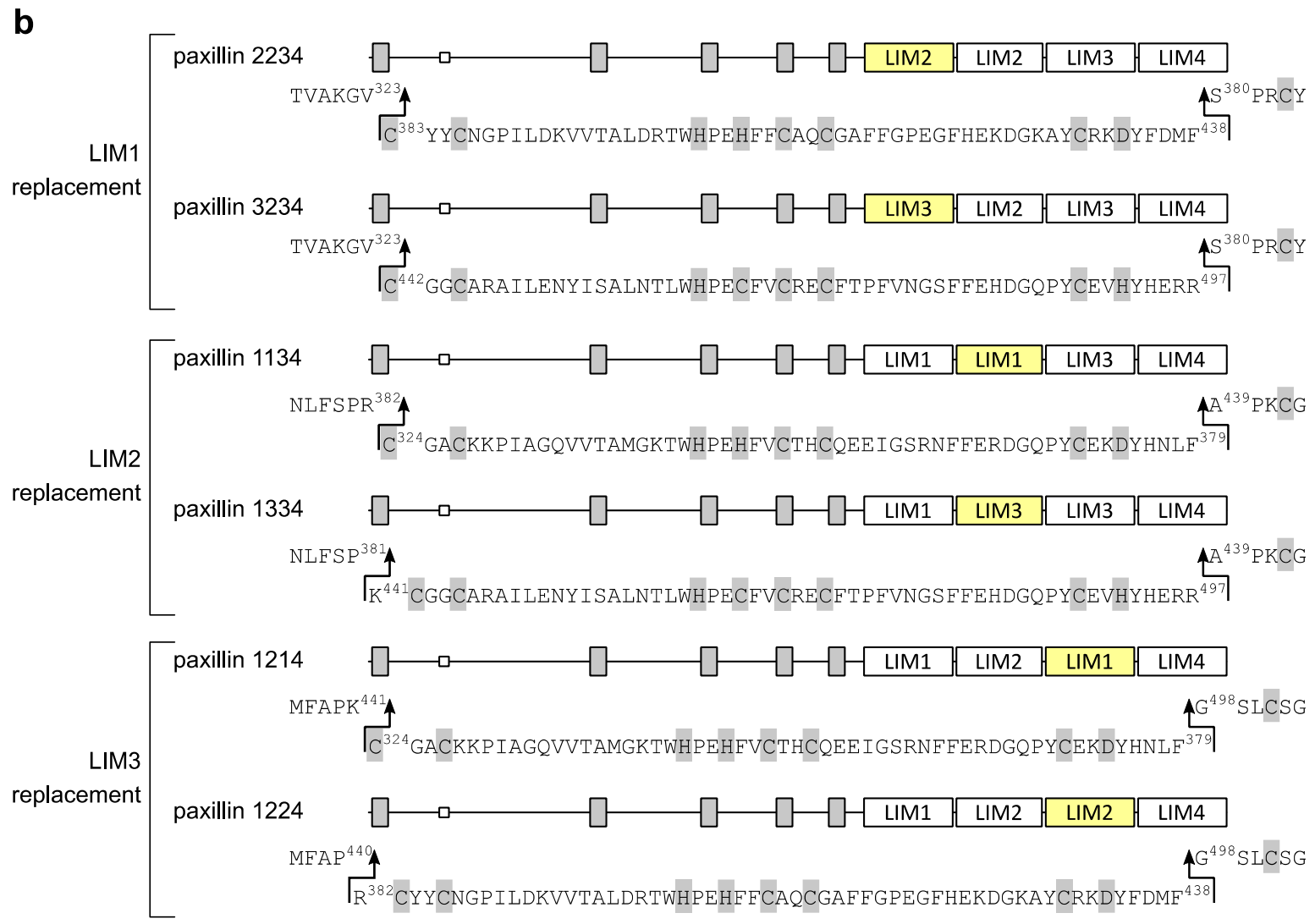

Supplementary Figure 4-LIM deletion and replacement strategies. (a) Schematic representation of the paxillin deletion mutants, showing the exact amino acid sequence deleted. (b) Schematic 
538 top line: replacements of LIM1, replacements of LIM2 and replacements of LIM3. The exact 539 boundaries used for the replacement are shown in the sequences. 
540 Supplementary Movies 1-3 - Live cell imaging of adhesions of NIH-3T3 fibroblasts.

- Movie 1 NIH-3T3 co-expressing $\beta 3^{\mathrm{WT}}$ _GFP and mCherry_paxillin.

- Movie 2 NIH-3T3 co-expressing $\beta 3^{\mathrm{VE} / \bar{Y} \mathrm{~A}}$ _GFP and mCherry_paxillin.

- Movie 3 NIH-3T3 co-expressing $\beta 3^{\mathrm{VE} / \mathrm{YA}}{ }_{-} \mathrm{CN}$ and mCherry_paxillin_CC.

544 mCh: mCherry; $\mathrm{CN}$ : citrine $\mathrm{N}$-terminal fragment; $\mathrm{CC}$ : citrine C-terminal fragment.

545 Supplementary Tables 1-4 - Statistical analysis.

546 - Table 1 Statistical analysis of BiFC experiments.

547 - Table 2 Statistical analysis of the integrin flow in adhesions.

548 - Table 3 Statistical analysis of photoactivation and FRAP experiments.

549 - Table 4 Statistical analysis of FA-isolation experiments. 


\section{Discussion}

551 A stepwise recruitment of paxillin to focal adhesions

552 The unique combination of molecular and cellular biological tools revealed that the LIM-dependent 553 localization of paxillin to FAs ${ }^{21}$ is the result of a synergistic binding to the PM and other FA 554 components. Although the N-terminal domain of paxillin is not contributing to the targeting 555 mechanism, it further stabilizes paxillin within FAs, when recruited there by the LIM-array. This 556 additional stabilization largely relies on phosphorylation of $\mathrm{Y}^{31}$ and/or $\mathrm{Y}^{118}$, which potentially

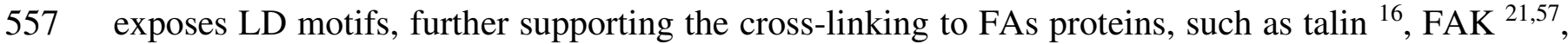
558 and/or vinculin ${ }^{10,21,58,59}$. Since the $\mathrm{N}$-terminal domain prolonged paxillin half-life over that of talin, 559 it is likely that the paxillin N-terminus interacts with neighboring integrin/talin units, as proposed in 560 the slanted fence model of FAs ${ }^{1}$.

\section{The focal adhesion targeting functions of the paxillin LIM1-LIM2-LIM3 array}

562 The LIM domain replacement strategy revealed the crucial role of paxillin LIM2 domain for its FA563 docking. As previously proposed for other LIM domain-proteins ${ }^{24,47}$, specific residues confer to distinct LIM domains exclusive functions and determine the selection of precise binding partners. Based on our results, we propose that the deletion or the structural disruption of a LIM domain ${ }^{21}$ alters the distance between the remaining domains, undermining multiple synergistic interactions of the LIMs-array with FAs, resulting in a cumulative binding defect. Indeed, the extension of the linker sequence between LIM domains can abrogate a proper protein localization to adhesions ${ }^{65}$, suggesting that serial LIM domain modules must have a given relative position to be properly anchored to this complex ${ }^{31}$.

571 Previous results supported an interaction of the paxillin LIM domains with the $\mathrm{Y}^{747}$ sidechain, situated in the membrane-proximal NPLY ${ }^{747}$ motif of $\beta 3$ integrin ${ }^{30,41-43,48}$. Although the dissociation rate of paxillin carrying an inactive LIM2 domain (paxillin 1334) was almost exactly that of paxillin wt dissociating from the $\beta 3^{\mathrm{VE} / \mathrm{YA}}$ integrin-containing adhesions (Fig. $2 \mathrm{~g}$,h and $6 \mathrm{~d}, \mathrm{e}$ ), we have no structural or biochemical proof that could indicate an interaction between $\mathrm{Y}^{747}$ of integrin and the LIM2 domain of paxillin. Interestingly, the binding of the LIM domains of the Enigma protein to NPLY-like peptides ${ }^{47,48}$ was prevented by mutations analogous to our $\mathrm{Y}^{747} \mathrm{~A}$ substitution ${ }^{47}$. An alternative binding mechanism of the paxillin LIM1-LIM2-LIM3 array to FAs could involve the recognition of tandem LIM domains by extended peptides of the $\beta 3$ integrin tail, in a complex similar to that proposed for LIM domain-containing transcription factors ${ }^{27}$. 
581 Recently, based on lysine cross-linking experiments, interactions between the N-terminal LD motifs 582 of paxillin and the $\mathrm{PH}$ and F0 domains of kindlin-2, as well as between the F0 domain and the paxillin 583 LIM3-LIM4 domains, have been identified and proposed to be responsible for paxillin recruitment to 584 FAs $^{36}$. However, none of these elements was strictly required for paxillin targeting and binding to 585 adhesions (Fig. 4b-e LIMs, Fig. 5 paxillin $\Delta$ LIM4, and Fig. 6f,g paxillin 1214 and paxillin 1224). 586 Even though the interaction with kindlin might be relevant for paxillin recruitment, it is probably not 587 the major factor for a stable docking to focal adhesions.

\section{LIM4 domain induces plasma membrane association of paxillin}

589 The local lipid composition of the PM influences the mobility of integrins, clustering and ligand 590 binding 4,45,66, and acts as an anchoring point for indispensable structural FA adapters, such as talin $591 \quad 67,68$ and kindlin ${ }^{69,70}$. Additionally, transiently recruited paxillin-binding FA-proteins, such as FAK, 592 require membrane interaction and a precise orientation for their activity ${ }^{71,72}$. Likewise, a PM593 dependent transient docking could be crucial for paxillin, which was identified as a membrane594 proximal protein by proximity biotinylation assays ${ }^{73}$. Moreover, Qin and co-workers recently solved 595 the NMR structure of the kindlin-2 F0/paxillin LIM4 complex ${ }^{38}$, which interaction could be part of 596 a kindlin-dependent mechanism of paxillin recruitment to the PM. Interestingly, such an interaction 597 with kindlin does not preclude the binding we propose between paxillin LIM4 and the PM. Consistent 598 with the hypothesis of a paxillin-PM interaction, we demonstrated paxillin palmitoylation (Fig. 7d). 599 However, the presence of 24 cysteines within the paxillin LIM domains and the limited possibility of 600 mutagenesis to preserve the double zinc-finger folding, hindered the identification of the paxillin 601 palmitoylation site(s). Nevertheless, the almost exclusive BiFC between the C-terminus of paxillin 602 and the PM-localized probe (Fig. 8b,c and Supplementary Fig.1d), accredited our hypothesis. 603 Importantly, the paxillin orientation within FAs relied on the LIMs-array, showing that the precise 604 organization of these domains matters for paxillin docking to FA.

\section{The $\beta 3$ integrin-talin-paxillin complex}

606 Within the elaborated network of (synergistic) bonds established among FA-proteins, paxillin is 607 among the most connected elements ${ }^{74}$. Together with talin and kindlin, paxillin belongs to the 608 structural subnet that connects integrin receptors to the actin cytoskeleton ${ }^{74}$. However, so far, it has 609 been only described as an adaptor or scaffold protein ${ }^{17}$. Nevertheless, the uncoupling of paxillin from 610 the binomial $\beta 3$ integrin/talin interaction $\left(\beta 3^{\mathrm{VE} / \mathrm{YA}}\right)$, produced FAs with structural defects, which could

611 be rescued by the reintroduction of the LIMs-array. Importantly, our data revealed that the 612 mechanically stable incorporation of paxillin into integrin-containing FAs is distinct from the 
613 mechanism of paxillin recruitment to the FA-microenvironment (Fig. 6h-k). This stepwise model of 614 paxillin recruitment and docking may involve a transient stabilization by the proposed LIM4-PM 615 interaction ${ }^{75}$. The latter could also account for the paxillin localization to $\mathrm{Mn}^{2+}$-induced integrin616 kindlin clusters in the absence of talin, which impaired maturation is reminiscent of the unstable $617 \beta 3^{\mathrm{VE} / \mathrm{YA}}$ adhesions phenotype ${ }^{37}$. Indeed, paxillin binding to the adhesion complex also reduces the 618 exchange rate of $\beta 3$ integrins between immature and F-actin coupled integrin-talin clusters ${ }^{45}$, as well 619 as the dynamic assembly/disassembly of FAs ${ }^{51}$.

620 Altogether, our data highlighted the need of comprehensive and integrating studies, based on 621 complementary approaches, to understand the structural interactions required for the physiological 622 functioning of focal adhesions, both at the level of the assembly of the focal adhesion, and its 623 mechanosensing and signal transduction. 


\section{Cell culture and transient transfection}

NIH-3T3 and Swiss-3T3 fibroblasts were grown at $37{ }^{\circ} \mathrm{C}\left(10 \% \mathrm{CO}_{2}\right)$ in DMEM with $4500 \mathrm{mg} / \mathrm{L}$ glucose (Sigma-Aldrich), supplemented with 10\% heat inactivated FBS (PANBiotech), 1\% penicillin-streptomycin (Gibco) and $2 \mathrm{mM}$ glutamine (Sigma-Aldrich). Transient transfections were performed 24 hours after seeding cells, with jetPRIME (Polyplus Transfection) according to the manufacturer's recommendations.

\section{2-bromopalmitate treatment and Acyl-Resin Assisted Capture (Acyl-RAC)}

Protein S-acylation was analyzed by the Acyl-RAC assay as described (Gadalla, Abrami, and Goot 2020), with some modifications. 4 hours after transfection, samples were split in two and treated with DMSO or 2-bromopalmitate at $60 \mu \mathrm{M}$. 24 hours after transfection, cells were trypsinized, washed with cold PBS and lysed in $300 \mu \mathrm{l}$ of lysis buffer (1.5\% Triton-X100, 25 mM HEPES, 1 mM EDTA in $8 \mathrm{M}$ urea solution supplemented with protease inhibitor cocktail). To block free SH groups with Smethyl methanethiosulfonate (MMTS, Sigma 64306), one volume of $2 \times$ blocking buffer (200 mM HEPES, 2 mM EDTA, 5\% SDS and 2.2\% MMTS in $8 \mathrm{M}$ urea solution supplemented with protease inhibitor cocktail) was added to the cell lysate and incubated, protected from light, for $5 \mathrm{~h}$ at $42{ }^{\circ} \mathrm{C}$ in a shaker $(600 \mathrm{rpm})$. Next, proteins were precipitated adding ice-cold methanol and chloroform (4/4/1 : protein mixture/methanol/chloroform), followed by centrifugation at $14000 \mathrm{rpm}$ for $15 \mathrm{~min}$ at $4{ }^{\circ} \mathrm{C}$. The protein pellet was broken using a needle and washed four times with $600 \mu \mathrm{l}$ of methanol. Airdried pellet was resuspended in $220 \mu 1$ of $5 \times$ binding buffer (100 mM HEPES, 5 mM EDTA, and 5\% SDS in $8 \mathrm{M}$ urea solution supplemented with protease inhibitor cocktail). Cell debris were removed by centrifugation at $14000 \mathrm{rpm}$ for $10 \mathrm{~min}$ at $4{ }^{\circ} \mathrm{C}$. $5 \%$ of the solubilized pellet was saved as Total Cell Lysate (TCE) to check for protein expression by western blotting. The remaining was diluted with $8 \mathrm{M}$ urea containing protease inhibitor cocktail, to reach a final volume of $1080 \mu \mathrm{l}$, and divided into two tubes. Thioester bonds of one aliquot were cleaved with $600 \mu 1$ of hydroxylamine (HA, Sigma 55459) freshly dissolved in water to $2.7 \mathrm{M}$ and adjusted to $\mathrm{pH} 7.4$. The control aliquot was treated with equal amount of 2M Tris-base. Thiopropyl Sepharose® 6B beads (Sigma T8387) were firstly activated for $1 \mathrm{~h}$ with $\mathrm{mqH}_{2} \mathrm{O}$, then resuspended in 3 volumes of binding buffer $(100 \mathrm{mM}$ HEPES, $1 \mathrm{mM}$ EDTA, and 1\% SDS in $8 \mathrm{M}$ urea solution supplemented with protease inhibitor cocktail) and finally added to protein samples (200 $\mu \mathrm{l}$ each). Samples were light-protected and incubated overnight at room temperature on a rotating wheel. Beads were then washed $5 \times$ in binding 
buffer and bound proteins eluted from the beads with SDS-PAGE sample buffer (140 mM SDS, 20\% glycerol, $25 \% \beta$-mercaptoethanol, and bromophenolblue) for $5 \mathrm{~min}$ at $95^{\circ} \mathrm{C}$.

\section{Photoactivation-based experiments}

658 Image acquisition and analysis were performed at the Bioimaging Core Facility, Faculty of Medicine, 659 University of Geneva. Briefly, transfected NIH-3T3 cells were cultured overnight on glass bottom 660 coverslips. 1 hour before imaging, culturing medium was replaced with F12 medium (Sigma661 Aldrich), supplemented with FBS, penicillin/streptomycin and glutamine. Photoactivation was 662 performed on a Nikon A1r confocal laser scanning microscope equipped with a 60x oil immersion 663 objective and a $37^{\circ} \mathrm{C}$ and $5 \% \mathrm{CO}_{2}$ incubation chamber. Laser wavelengths of $488 \mathrm{~nm}$ and $561 \mathrm{~nm}$ 664 were used to acquire three pictures at $5 \mathrm{sec}$ intervals before photoactivation and 1 frame every $2 \mathrm{sec}$ 665 for 3 min after photoactivation. Excitation of photoactivatable GFP molecules was achieved by means 666 of a $405 \mathrm{~nm}$ laser (10\% power), on a single ROI matching the size of an mCherry-positive focal 667 adhesion. Using Imaris combined with MatLab scripts, we identified the area of photoactivation, 668 automatically repositioned it in case of lateral shift according to the mCherry signal and extracted the 669 mean green intensity within the ROI for each time point. The first three time points, corresponding 670 to the background, were averaged and subtracted from the full-time course. The intensity of the first 671 acquisition after photoactivation was set to " $100 \%$ intensity", and all other values were calculated as 672 ratio. For each photoactivation time series, a constrained double decaying exponentials model 673 (decreasing form) with the formula $\left(\bar{F}_{0}-c\right)\left(k_{\text {fast }} e^{-\lambda_{\text {fast }} \cdot t}+\left(\bar{F}_{0}-k_{\text {fast }}\right) e^{-\lambda_{\text {slow }} \cdot t}\right)+c$ was use to 674 fit the data points. For each of them, the half-life was defined as the time needed to lose $50 \%$ of the 675 intensity at time 0 , shown in the box and whisker plot, and used for statistical comparison. The same 676 constrained double decaying exponentials model was also used to fit the average data, offering a visual inspection of the goodness of the fit (theoretical model).

FRAP was conducted similarly to photoactivation experiments. However, seeding of transfected cells on coverslips was performed 4 hours after transfection to avoid prior irreversible binding of paxillin 681 to the PM via SpyTag/SpyCatcher. Time intervals of acquisition were maintained, while the photoactivation step was replaced by 3 rounds of scanning using the laser wavelength of $561 \mathrm{~nm}$ at $100 \%$ power, which efficiently bleached the mCherry fluorophore localized within the selected adhesion. The residual fluorescence was subtracted from the entire time curse, the first three acquisitions, corresponding to the mCherry level prior to bleaching, were averaged and set to $100 \%$ intensity, while all other values were calculated as ratio. Individual replicates were fitted with a 
687 constrained double exponentials model (increasing form) with the formula $\bar{F}_{\text {fast }}\left(1-e^{-\lambda_{\text {fast }} \cdot t}\right)+$ $688 \bar{F}_{\text {slow }}\left(1-e^{-\lambda_{\text {slow }} \cdot t}\right)$. The theoretical half-lives thereof calculated were presented in box and whisker 689 plots and used for statistical comparison.

690 Isolation of focal adhesions, immunostaining and TIRF microscopy

691 After culturing cells overnight on coverslips, FAs were isolated by means of a protocol previously 692 described (Kuo et al. 2012) and immediately fixed with 4\% PFA/PBS. For immunostaining, isolated 693 complexes were blocked for $30 \mathrm{~min}$ with a solution of PBS containing $1 \%$ BSA, incubated for $60 \mathrm{~min}$ 694 with the primary antibody and subsequently for $45 \mathrm{~min}$ with the corresponding secondary antibody, 695 both diluted in the same buffer. Images were acquired on a Nikon Eclipse Ti equipped with a 100x 696 oil immersion objective. TIRF angle and lasers intensities were kept constant for all experiments. For 697 each image acquired, a custom Metamorph journal was used to identify adhesions on the green 698 channel and subsequently to extract the mean intensity for each of the laser wavelengths used. The 699 quantification of the capacity of cytosolic proteins to be retained in the isolated FAs was expressed 700 in respect to the residual integrin fluorescence, thus as a red/green ratio. In the case of antibody 701 staining, to compensate for possible variations among experiments, the mean red/green ratio in $\beta 3^{\mathrm{WT}}$ 702 integrin adhesions was always considered equal to 1, and therefore all the measures expressed in 703 respect to it.

\section{Bimolecular Fluorescence Complementation (BiFC)}

705 The citrine fluorophore (FPbase ID VR7EN), generated by the $\mathrm{Q}^{69} \mathrm{M}$ mutation within the YFP 706 sequence, was obtained as previously described (Paulhe et al. 2009). The N-terminal (CN, aa 1-173) 707 and the C-terminal (CC, aa 174-239) fragments were cloned upstream or downstream the coding 708 sequences of our proteins of interest.

709 For BiFC assays, transfected cells were seeded on glass coverslips 4 hours after transfection and fixed 710 with 4\% PFA/PBS 48 hours later. Images were acquired on a Nikon Eclipse Ti equipped with a 100x 711 oil immersion objective. TIRF angle and lasers intensities were kept constant for all experiments. 712 Images were processed with Metamorph, as explained above for isolated FAs, with the only 713 difference that adhesions were identified in virtue of their red fluorescence instead of the green one. 714 For the quantification of the BiFC efficiency, the first step consisted of determining the green 715 background, corresponding to the mean green intensity in mCherry-positive FAs measured by 716 applying the just described analysis to NIH-3T3 and Swiss-3T3 cells only expressing the 717 CN_paxillin_mCherry construct. This value was then subtracted from the mean green intensity in 718 adhesions of each image acquired upon co-expression of pair of BiFC probes. The resulting green 
value was divided by the red mean intensity in adhesions of the same image. Finally, this ratio was compared to the one obtained by expressing solely the corresponding paxillin/LIMs construct presenting the full-length citrine in place of the $\mathrm{CN} / \mathrm{CC}$ fragment (and analyzed in the same way) considered to be the maximal, $100 \%$, efficiency that could be reached.

\section{Integrin flow in adhesions}

As for BiFC assays, cells were seeded on coverslips 4 hours after transfection. Two days later, the culturing medium was replaced with F12 medium (Sigma-Aldrich), supplemented with FBS, penicillin/streptomycin and glutamine, and cells were imaged by means of a Nikon A1r confocal laser scanning microscope equipped with a $60 \mathrm{x}$ oil immersion objective and a $37{ }^{\circ} \mathrm{C}$ and $5 \% \mathrm{CO}_{2}$ incubation chamber. Laser wavelengths of $488 \mathrm{~nm}$ and $561 \mathrm{~nm}$ were used to acquire 1 frame every minute for a total of 10 minutes. Adhesions of a given cell, were segmented frame by frame, using the neurite function of Metamorph applied on the (mCherry) paxillin-derived fluorescence channel. Thereafter, Matlab was used to correct the possible translational drifts of cells, and their respective adhesion masks, occurring during the time course of the acquisition. Then background subtraction, followed by a non-local means filter was used to diminish the contribution of spatially stationary areas, i.e. mainly outside of adhesions. Dense optical flow was estimated for all the pixels in the frames, using the Farnebäck's method ${ }^{76}$. The velocity of the adhesions flow was computed as the average magnitude of the flow within the adhesion mask between two following frames.

\section{Statistics}

BiFC data did not consistently satisfy normal distribution and therefore statistical comparison was performed using the Wilcoxon test based on ranks. For comparison among more than two groups the Kruskal-Wallis extension was applied. Significant differences among proteins retained in isolated FAs were analogously evaluated. Differences of integrins flows in adhesions among time points or between samples were assessed by one-way Anova test. The half-lives calculated from FLAP data were subject to one-way Anova test, while for half-lives calculated from FRAP experiments the Kruskal-Wallis test was employed. All multiple comparisons were followed by Tukey-Kramer posthoc correction. The nomenclature used in figures is based on the following criteria: ns (not significant) $p>0.05$ * $^{*}, p<0.05 ; * *, p<0.01$; ***, $p<0.001$; ****, $p<0.0001$. For each experiment, sample size, complete statistical analysis and $p$ values are provided in tables in supplementary dataset. All experiments were repeated at least three times and no repeated measurements were performed. For all box and whisker plots presented in this manuscript the box shows the median, $25^{\text {th }}$ and $75^{\text {th }}$ percentiles, while the whiskers the maximum and minimum values. Outliers are indicated with the 
symbol $x$. FLAP data are shown as mean intensity \pm standard error of the mean (experimental) or constrained double decaying exponentials model \pm standard error of the mean (theoretical dissociation model). For FRAP experiments, the mean value for each time point is represented with a dot, the standard error of the mean with a colored background and the fitting of the double exponential function to the mean values with black a curve.

\section{cDNAs and site-directed mutagenesis}

The cDNA encoding the full-length, wild-type and mutant, mouse $\beta 3$ integrins were obtained from the previously described integrin fusion proteins (Pinon et al. 2014). The linker, coding for the sequence DGSPVAT and containing an AgeI site, was introduced in between the integrin sequence (Uniprot identifier O54890-1) and the fluorescent protein (either GFP, mCherry, PA-GFP or citrine fragments).

Paxillin fusion proteins were expressed in a cytomegalovirus promoter-driven pcDNA3 vector. All residues of paxillin recombinant proteins were numbered according to the human paxillin $\alpha$ (Uniprot identifier P49023-2). Paxillin N-terminus and LIMs constructs were obtained by the cloning of a PCR-amplified fragment of full-length paxillin, either corresponding to the first 322 amino acids or to the C-terminal sequence starting from residue 317. Paxillin LIM domain deletion mutants were obtained by overlap extension PCR, producing the deletion shown in Supplementary Fig. 4a, and here summarized. Paxillin $\Delta$ LIM1: deletion of aa 322-380; paxillin $\triangle$ LIM2: deletion of aa 381-439; paxillin $\triangle$ LIM3 deletion of aa 441-499; paxillin $\triangle$ LIM4 truncation from aa 500. Point mutations were introduced by site-directed mutagenesis. For the LIM domain replacement strategy, we either used serial overlap extension PCRs with degenerated primers or cloned a de novo-synthetized nucleotide sequence, coding for the LIM domain of interest but with a degenerated codons compared to the original one. The amino acid sequence of the final recombinant proteins is shown in Supplementary Fig. 4b. In summary, in paxillin 2234 the aa 324-379 were replaced by the sequence found at aa 383438; in paxillin 3234 the aa 324-379 were replaced by the sequence found at aa 442-497; in paxillin 1134 the aa 383-438 were replaced by the sequence found at aa 324-379; in paxillin 1334 the aa 382438 were replaced by the sequence found at aa 441-497; in paxillin 1214 the aa 442-497 were replaced by the sequence found at aa 324-379; and in paxillin 1224 the aa 441-497 were replaced by the sequence found at aa 382-438. The C-terminally tagged paxillins (paxillin_PA-GFP and paxillin proteins for BiFC and SpyTag/SpyCatcher assays) were obtained by removing the stop codon in a PCR step, and then joining the sequence coding for one of the tags via a linker coding for a GPVAT peptide, containing an AgeI site. 
783 The PM-anchored proteins were obtained by sequential cloning of the sequences coding for the PM784 targeting peptide of Lck (MGCVCSSNPEL) and the citrine fragment (either CN or CC), or the 785 SpyCatcher, into a pCDNA3 vector. The plasma membrane-targeting sequence was recovered from 786 the Lck-mScarlet-I (98821; Addgene; deposited by D. Gadella) ${ }^{77}$, a gift from N. Gauthier (IFOM, 787 the FIRC Institute for Molecular Oncology, Milan, Italy).

788 PA-GFP_talin was obtained by replacing the ECFP with the PA-GFP fluorophore, in the chimeric 789 full-length construct previously described (Saltel et al. 2009).

790 The amino acids compositions of the fluorescent proteins can be found in the FPbase database, at the 791 following IDs. Photoactivatable GFP (PA-GFP): FPbase ID 7QYHY (gift from Christoph Ballestrem, 792 Wellcome Trust Centre for Cell-Matrix Research, University of Manchester, Manchester, UK) ${ }^{78}$; 793 mCherry: FPbase ID ZERB6; citrine: FPbase ID VR7EN. SpyTag/SpyCatcher003 sequences can be 794 found in the Addgene repository ${ }^{64}$.

795 DNA sequence analysis was performed for all constructs and mutants to ensure error-free 796 amplification and correct base replacement.

\section{Protein extraction and western blotting}

798 NIH-3T3 transiently transfected were trypsinized and lysed with Ripa buffer (150 mM NaCl, $5 \mathrm{mM}$ 799 EDTA, 25 mM Tris- $\mathrm{HCl}$ pH 7.6, 1\% Triton X-100, 1\% sodium deoxycholate, 0.1\% SDS, $1 \mathrm{mM}$ $800 \mathrm{PMSF}$, and $1 \mu \mathrm{g} / \mathrm{ml}$ chymostatin, leupeptin, antipain, and pepstatin; all obtained from Sigma-Aldrich) 801 during $30 \mathrm{~min}$ on ice. Cell lysates were then cleared by a $30 \mathrm{~min}$ centrifugation at $14000 \mathrm{rpm}$ and at $8024{ }^{\circ} \mathrm{C}$, supplemented with SDS sample buffer $4 \times(140 \mathrm{mM}$ SDS, 20\% glycerol, 25\% $\beta$ 803 mercaptoethanol, and bromophenolblue) and boiled for $5 \mathrm{~min}$. Samples were resolved by SDS-PAGE 804 and transferred to nitrocellulose membranes (GE Healthcare). Membrane were blocked in 5\% (w/v) 805 BSA (A1391 Applichem) diluted in TBST buffer (0.5 m Tris-base, $1.5 \mathrm{M} \mathrm{NaCl}, 0.1 \%$ Tween 20, pH 806 7.6), then incubated with primary antibody followed by horseradish peroxidase (HRP)-conjugated 807 secondary antibody, both diluted in 5\% BSA TBST buffer. Staining were revealed using Advansta 808 ECL Quantum (K-12042) or Sirius (K-12043) substrates.

\section{Reagents}

810 Purified mouse monoclonal anti-paxillin (BD Biosciences 610051). Mouse monoclonal anti-vinculin

811 (Sigma-Aldrich V9131). Rabbit polyclonal anti-caveolin-1 (Santa Cruz Biotechnology sc-894). 812 Rabbit polyclonal anti-mCherry (BioVision 5993-30T). Alexa Fluor 555 anti-mouse (ThermoFisher 
813 A-31570). Alexa Fluor 633 anti-mouse (ThermoFisher A-21050). HRP-conjugated anti-mouse 814 (Jackson). HRP-conjugated anti-rabbit (Jackson). 


\section{Acknowledgments}

816 We are grateful to Dr. Sunil Dogga for introducing us to the Acyl-RAC technique and to Dr. Patricia 817 Vazquez for her contribution in the initial phase of this work. We thank Prof. Vesa Hytönen, Latifeh 818 Azizi and Paula Turkki for helpful discussions and providing the SpyTag/SpyCatcher cDNAs. We 819 are grateful to all the members of the Bioimaging core facility of the Centre Médical Universitaire 820 for help in microscopy and image analysis. This work was supported by the Swiss National Science 821 Foundation, grants 31003A_166384 and 310030_185261. The authors declare no competing financial 822 interests.

\section{Author contributions}

824 MR and BWH designed the DNA constructs and experiments. MR conducted the experiments. NL 825 designed the strategy to analyze the photoactivation and FRAP data and the integrin flow in 826 adhesions. MR and BWH wrote the manuscript. All authors accepted the final version of the 827 manuscript.

\section{Data availability}

829 All microscopy images shown or analyzed in this work are deposited at University of Geneva and are 830 available upon request.

\section{Code availability}

832 Matlab and Metamorph codes were developed in-house at the Bioimaging Core Facility, Faculty of 833 Medicine, University of Geneva, and here deposited. Codes are available upon request. 


\section{References}

836 1. Bachmann, M., Kukkurainen, S., Hytonen, V.P. \& Wehrle-Haller, B. Cell Adhesion by

2. Kechagia, J.Z., Ivaska, J. \& Roca-Cusachs, P. Integrins as biomechanical sensors of the microenvironment. Nat Rev Mol Cell Biol 20, 457-473 (2019).

3. Bachmann, M. et al. Induction of ligand promiscuity of alphaVbeta3 integrin by mechanical force. J Cell Sci 133(2020).

4. Wehrle-Haller, B. Structure and function of focal adhesions. Curr Opin Cell Biol 24, 116-24 (2012).

5. Yu, C.H., Luo, W. \& Sheetz, M.P. Spatial-temporal reorganization of activated integrins. Cell Adh Migr 6, 280-4 (2012).

6. Roca-Cusachs, P., Gauthier, N.C., Del Rio, A. \& Sheetz, M.P. Clustering of alpha(5)beta(1) integrins determines adhesion strength whereas alpha(v)beta(3) and talin enable mechanotransduction. Proc Natl Acad Sci U S A 106, 16245-50 (2009).

7. von Wichert, G., Haimovich, B., Feng, G.S. \& Sheetz, M.P. Force-dependent integrincytoskeleton linkage formation requires downregulation of focal complex dynamics by Shp2. EMBO J 22, 5023-35 (2003).

8. Webb, D.J. et al. FAK-Src signalling through paxillin, ERK and MLCK regulates adhesion disassembly. Nat Cell Biol 6, 154-61 (2004).

9. Changede, R., Xu, X., Margadant, F. \& Sheetz, M.P. Nascent Integrin Adhesions Form on All Matrix Rigidities after Integrin Activation. Dev Cell 35, 614-621 (2015).

10. Case, L.B. et al. Molecular mechanism of vinculin activation and nanoscale spatial organization in focal adhesions. Nat Cell Biol 17, 880-92 (2015).

11. Kalappurakkal, J.M. et al. Integrin Mechano-chemical Signaling Generates Plasma Membrane Nanodomains that Promote Cell Spreading. Cell 177, 1738-1756 e23 (2019).

12. Weng, Z., Taylor, J.A., Turner, C.E., Brugge, J.S. \& Seidel-Dugan, C. Detection of Src homology 3-binding proteins, including paxillin, in normal and v-Src-transformed Balb/c 3T3 cells. J Biol Chem 268, 14956-63 (1993).

13. Alam, T., Alazmi, M., Gao, X. \& Arold, S.T. How to find a leucine in a haystack? Structure, ligand recognition and regulation of leucine-aspartic acid (LD) motifs. Biochem J 460, 31729 (2014).

14. Pasapera, A.M., Schneider, I.C., Rericha, E., Schlaepfer, D.D. \& Waterman, C.M. Myosin II activity regulates vinculin recruitment to focal adhesions through FAK-mediated paxillin phosphorylation. J Cell Biol 188, 877-90 (2010).

15. Tumbarello, D.A., Brown, M.C. \& Turner, C.E. The paxillin LD motifs. FEBS Lett 513, 1148 (2002).

16. Zacharchenko, T. et al. LD Motif Recognition by Talin: Structure of the Talin-DLC1 Complex. Structure 24, 1130-41 (2016).

17. Deakin, N.O. \& Turner, C.E. Paxillin comes of age. J Cell Sci 121, 2435-44 (2008).

18. Frank, S.R. \& Hansen, S.H. The PIX-GIT complex: a G protein signaling cassette in control of cell shape. Semin Cell Dev Biol 19, 234-44 (2008).

19. Turner, C.E. Paxillin and focal adhesion signalling. Nat Cell Biol 2, E231-6 (2000).

20. Webb, D.J. et al. Paxillin phosphorylation sites mapped by mass spectrometry. J Cell Sci 118, 4925-9 (2005).

21. Brown, M.C., Perrotta, J.A. \& Turner, C.E. Identification of LIM3 as the principal determinant of paxillin focal adhesion localization and characterization of a novel motif on paxillin directing vinculin and focal adhesion kinase binding. J Cell Biol 135, 1109-23 (1996).

22. Kadrmas, J.L. \& Beckerle, M.C. The LIM domain: from the cytoskeleton to the nucleus. Nat Rev Mol Cell Biol 5, 920-31 (2004). 
23. Velyvis, A., Yang, Y., Wu, C. \& Qin, J. Solution structure of the focal adhesion adaptor PINCH LIM1 domain and characterization of its interaction with the integrin-linked kinase ankyrin repeat domain. J Biol Chem 276, 4932-9 (2001).

24. Arber, S. \& Caroni, P. Specificity of single LIM motifs in targeting and LIM/LIM interactions in situ. Genes Dev 10, 289-300 (1996).

25. Chiswell, B.P. et al. Structural basis of competition between PINCH1 and PINCH2 for binding to the ankyrin repeat domain of integrin-linked kinase. J Struct Biol 170, 157-63 (2010).

26. Gadd, M.S. et al. A structural basis for the regulation of the LIM-homeodomain protein islet 1 (Isl1) by intra- and intermolecular interactions. J Biol Chem 288, 21924-35 (2013).

27. Matthews, J.M. \& Potts, J.R. The tandem beta-zipper: modular binding of tandem domains and linear motifs. FEBS Lett 587, 1164-71 (2013).

28. Schmeichel, K.L. \& Beckerle, M.C. The LIM domain is a modular protein-binding interface. Cell 79, 211-9 (1994).

29. Vaynberg, J. et al. Non-catalytic signaling by pseudokinase ILK for regulating cell adhesion. Nat Commun 9, 4465 (2018).

30. Sawada, Y. \& Sheetz, M.P. Force transduction by Triton cytoskeletons. J Cell Biol 156, 60915 (2002).

31. Schiller, H.B. \& Fassler, R. Mechanosensitivity and compositional dynamics of cell-matrix adhesions. EMBO Rep 14, 509-19 (2013).

32. Schiller, H.B., Friedel, C.C., Boulegue, C. \& Fassler, R. Quantitative proteomics of the integrin adhesome show a myosin II-dependent recruitment of LIM domain proteins. EMBO Rep 12, 259-66 (2011).

33. Dawid, I.B., Breen, J.J. \& Toyama, R. LIM domains: multiple roles as adapters and functional modifiers in protein interactions. Trends Genet 14, 156-62 (1998).

34. Smith, M.A. et al. LIM domains target actin regulators paxillin and zyxin to sites of stress fiber strain. PLoS One 8, e69378 (2013).

35. Wade, R. \& Vande Pol, S. Minimal features of paxillin that are required for the tyrosine phosphorylation of focal adhesion kinase. Biochem J 393, 565-73 (2006).

36. Bottcher, R.T. et al. Kindlin-2 recruits paxillin and Arp2/3 to promote membrane protrusions during initial cell spreading. J Cell Biol 216, 3785-3798 (2017).

37. Theodosiou, M. et al. Kindlin-2 cooperates with talin to activate integrins and induces cell spreading by directly binding paxillin. Elife 5, e10130 (2016).

38. Zhu, L. et al. Structural Basis of Paxillin Recruitment by Kindlin-2 in Regulating Cell Adhesion. Structure 27, 1686-1697 e5 (2019).

39. Zhang, $X$. et al. Talin depletion reveals independence of initial cell spreading from integrin activation and traction. Nat Cell Biol 10, 1062-8 (2008).

40. Wixler, V. et al. The LIM-only protein DRAL/FHL2 binds to the cytoplasmic domain of several alpha and beta integrin chains and is recruited to adhesion complexes. $J$ Biol Chem 275, 33669-78 (2000).

41. Soto-Ribeiro, M. et al. beta1D integrin splice variant stabilizes integrin dynamics and reduces integrin signaling by limiting paxillin recruitment. J Cell Sci 132(2019).

42. Hytonen, V.P. \& Wehrle-Haller, B. Mechanosensing in cell-matrix adhesions - Converting tension into chemical signals. Exp Cell Res 343, 35-41 (2016).

43. Pinon, P. et al. Talin-bound NPLY motif recruits integrin-signaling adapters to regulate cell spreading and mechanosensing. J Cell Biol 205, 265-81 (2014).

44. Wegener, K.L. et al. Structural basis of integrin activation by talin. Cell 128, 171-82 (2007).

45. Cluzel, C. et al. The mechanisms and dynamics of (alpha)v(beta)3 integrin clustering in living cells. J Cell Biol 171, 383-92 (2005).

46. Hytonen, V.P. \& Wehrle-Haller, B. Protein conformation as a regulator of cell-matrix adhesion. Phys Chem Chem Phys 16, 6342-57 (2014). 
47. Wu, R. et al. Specificity of LIM domain interactions with receptor tyrosine kinases. J Biol Chem 271, 15934-41 (1996).

48. Wu, R.Y. \& Gill, G.N. LIM domain recognition of a tyrosine-containing tight turn. J Biol Chem 269, 25085-90 (1994).

49. Pfaff, M. \& Jurdic, P. Podosomes in osteoclast-like cells: structural analysis and cooperative roles of paxillin, proline-rich tyrosine kinase 2 (Pyk2) and integrin alphaVbeta3. J Cell Sci 114, 2775-86 (2001).

50. Schaller, M.D., Otey, C.A., Hildebrand, J.D. \& Parsons, J.T. Focal adhesion kinase and paxillin bind to peptides mimicking beta integrin cytoplasmic domains. J Cell Biol 130, 11817 (1995).

51. Deakin, N.O. \& Turner, C.E. Distinct roles for paxillin and Hic-5 in regulating breast cancer cell morphology, invasion, and metastasis. Mol Biol Cell 22, 327-41 (2011).

52. Kerppola, T.K. Bimolecular fluorescence complementation (BiFC) analysis as a probe of protein interactions in living cells. Annu Rev Biophys 37, 465-87 (2008).

53. Machiyama, H. et al. Displacement of p130Cas from focal adhesions links actomyosin contraction to cell migration. J Cell Sci 127, 3440-50 (2014).

54. Morell, M., Espargaro, A., Aviles, F.X. \& Ventura, S. Detection of transient protein-protein interactions by bimolecular fluorescence complementation: the Abl-SH3 case. Proteomics 7, 1023-36 (2007).

55. Kuo, J.C., Han, X., Yates, J.R., 3rd \& Waterman, C.M. Isolation of focal adhesion proteins for biochemical and proteomic analysis. Methods Mol Biol 757, 297-323 (2012).

56. Legerstee, K., Geverts, B., Slotman, J.A. \& Houtsmuller, A.B. Dynamics and distribution of paxillin, vinculin, zyxin and VASP depend on focal adhesion location and orientation. Sci Rep 9, 10460 (2019).

57. Choi, C.K., Zareno, J., Digman, M.A., Gratton, E. \& Horwitz, A.R. Cross-correlated fluctuation analysis reveals phosphorylation-regulated paxillin-FAK complexes in nascent adhesions. Biophys J 100, 583-592 (2011).

58. Turner, C.E. et al. Paxillin LD4 motif binds PAK and PIX through a novel 95-kD ankyrin repeat, ARF-GAP protein: A role in cytoskeletal remodeling. J Cell Biol 145, 851-63 (1999).

59. Turner, C.E., Glenney, J.R., Jr. \& Burridge, K. Paxillin: a new vinculin-binding protein present in focal adhesions. J Cell Biol 111, 1059-68 (1990).

60. Parat, M.O. \& Fox, P.L. Palmitoylation of caveolin-1 in endothelial cells is post-translational but irreversible. J Biol Chem 276, 15776-82 (2001).

61. Kanchanawong, P. et al. Nanoscale architecture of integrin-based cell adhesions. Nature 468, 580-4 (2010).

62. Zlatkine, P., Mehul, B. \& Magee, A.I. Retargeting of cytosolic proteins to the plasma membrane by the Lck protein tyrosine kinase dual acylation motif. J Cell Sci 110 ( Pt 5), 6739 (1997).

63. Bertolucci, C.M., Guibao, C.D. \& Zheng, J. Structural features of the focal adhesion kinasepaxillin complex give insight into the dynamics of focal adhesion assembly. Protein Sci 14, 644-52 (2005).

64. Keeble, A.H. et al. Approaching infinite affinity through engineering of peptide-protein interaction. Proc Natl Acad Sci U S A (2019).

65. Petit, M.M., Meulemans, S.M. \& Van de Ven, W.J. The focal adhesion and nuclear targeting capacity of the LIM-containing lipoma-preferred partner (LPP) protein. J Biol Chem 278, 2157-68 (2003).

66. Eich, C. et al. Changes in membrane sphingolipid composition modulate dynamics and adhesion of integrin nanoclusters. Sci Rep 6, 20693 (2016).

67. Goksoy, E. et al. Structural basis for the autoinhibition of talin in regulating integrin activation. Mol Cell 31, 124-33 (2008). 
68. Saltel, F. et al. New PI(4,5)P2- and membrane proximal integrin-binding motifs in the talin head control beta3-integrin clustering. J Cell Biol 187, 715-31 (2009).

69. Liu, J. et al. Structural basis of phosphoinositide binding to kindlin-2 protein pleckstrin homology domain in regulating integrin activation. J Biol Chem 286, 43334-42 (2011).

70. Liu, Y., Zhu, Y., Ye, S. \& Zhang, R. Crystal structure of kindlin-2 PH domain reveals a conformational transition for its membrane anchoring and regulation of integrin activation. Protein Cell 3, 434-40 (2012).

71. Goni, G.M. et al. Phosphatidylinositol 4,5-bisphosphate triggers activation of focal adhesion kinase by inducing clustering and conformational changes. Proc Natl Acad Sci U S A 111, E3177-86 (2014).

72. Herzog, F.A., Braun, L., Schoen, I. \& Vogel, V. Structural Insights How PIP2 Imposes Preferred Binding Orientations of FAK at Lipid Membranes. J Phys Chem B 121, 3523-3535 (2017).

73. Dong, J.M. et al. Proximity biotinylation provides insight into the molecular composition of focal adhesions at the nanometer scale. Sci Signal 9, rs4 (2016).

74. Zaidel-Bar, R., Itzkovitz, S., Ma'ayan, A., Iyengar, R. \& Geiger, B. Functional atlas of the integrin adhesome. Nat Cell Biol 9, 858-67 (2007).

75. Wolfenson, $\mathrm{H}$. et al. A role for the juxtamembrane cytoplasm in the molecular dynamics of focal adhesions. PLoS One 4, e4304 (2009).

76. Farnebäck, G. Two-Frame Motion Estimation Based on Polynomial Expansion, 363-370 (2003).

77. Chertkova, A.O. et al. Robust and Bright Genetically Encoded Fluorescent Markers for Highlighting Structures and Compartments in Mammalian Cells. bioRxiv, 160374 (2020).

78. Stutchbury, B., Atherton, P., Tsang, R., Wang, D.Y.\& Ballestrem, C. Distinct focal adhesion protein modules control different aspects of mechanotransduction. J Cell Sci 130, 1612-1624 (2017). 


\section{Figures}
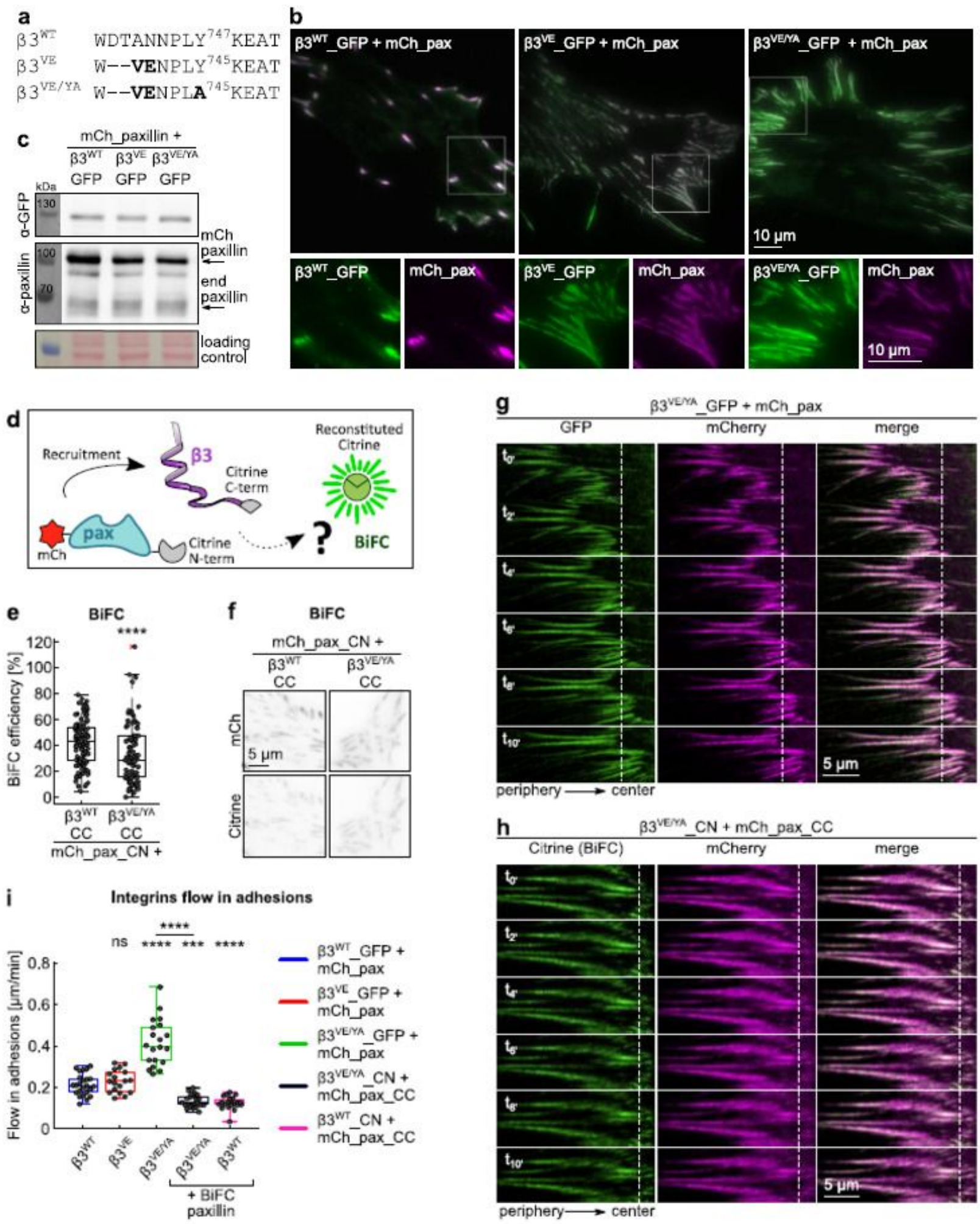

\section{Figure 1}

Morphology and dynamics of wild-type and mutant $\beta 3$ _GFP integrin-positive adhesions. (a) Sequence alignment of $\beta 3$ integrins, wt and mutants, at the NPLY747 motif. The high-affinity talin-binding chimeric $\beta 3$ integrins were obtained by introducing the VE motif from layilin. Bold indicates mutated residues. $(b, c)$ 
NIH-3T3 cells transiently transfected with 33 _GFP integrins, wild-type or mutants, and mCherry_paxillin. (b) Representative TIRF images of cells fixed 24 hoursafter spreading on glass coverslips in serum containing medium and (c) recombinant protein expression assessed by western blotting against GFP and total paxillin. Loading control: Ponceau staining. (d) Schematic representation of the BiFC assay used to evaluate paxillin recruitment to $\beta 3$ integrins. (e,f) BiFC upon co-expression of mCherry_paxillin_CN and $33 W T$ CCC or B3VE/YA_CC in NIH-3T3 fibroblasts. (e) Quantification of the BiFC efficiency. Statistical analysis is provided in Supplementary Table 1. (f) Representative TIRF images. (g,h) Kymographs representative of the displacement of $\beta 3 \mathrm{VE} / \mathrm{YA}$-containing adhesions, over a time period of 10 minutes, in the absence ( $\mathrm{g}$ )and in the presence $(\mathrm{h}$ ) of mechanically (BiFC) coupled paxillin. (i) Quantification of the $\beta 3$ integrins flow in adhesions, expressed as mean displacement over time $(\mu \mathrm{m} / \mathrm{min})$ per replicate.

Statistical 154 analysis is provided in Supplementary Table 2. 
a $\quad \beta 3$ integrins dissociation curves

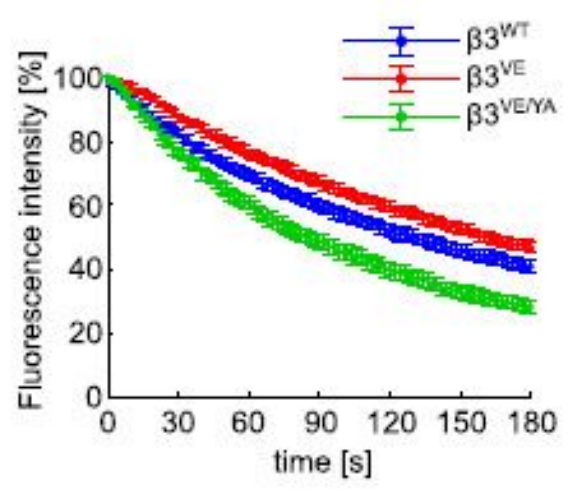

d Talin dissociation curves

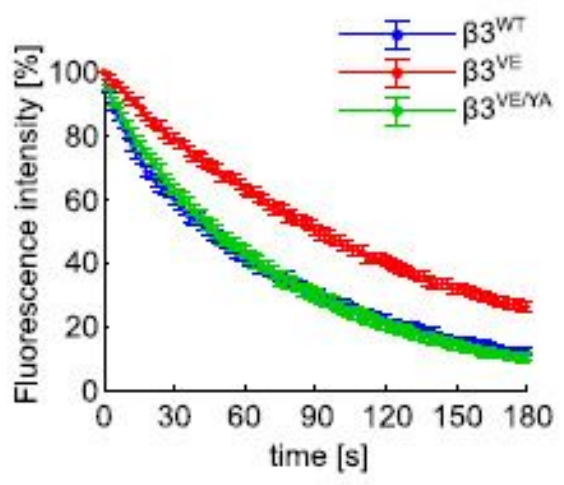

g Paxillin dissociation curves

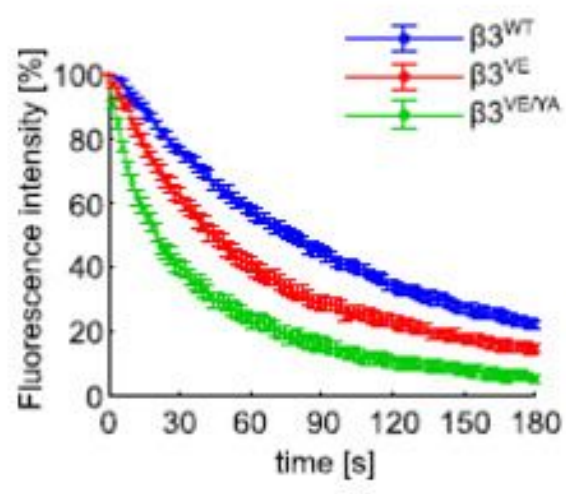

b Integrins half-lives

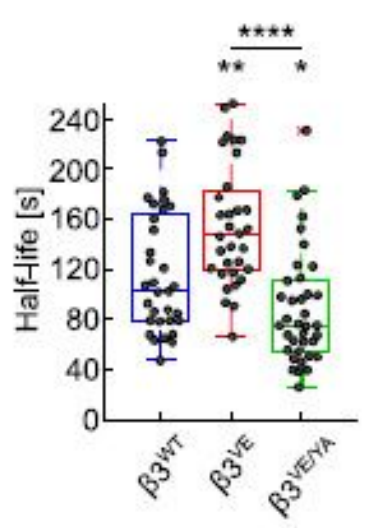

e

Talin half-life

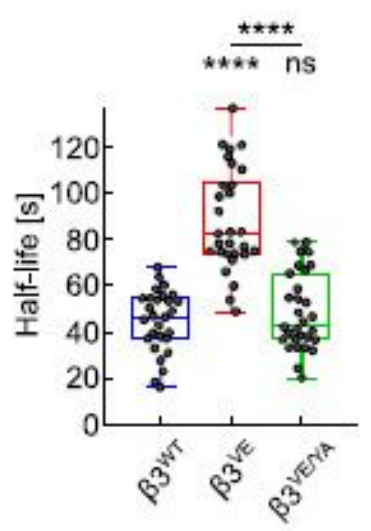

h Paxillin half-life

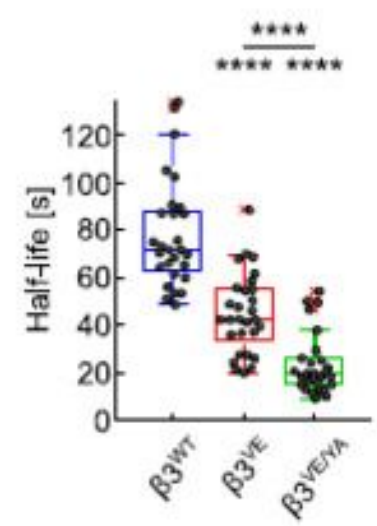

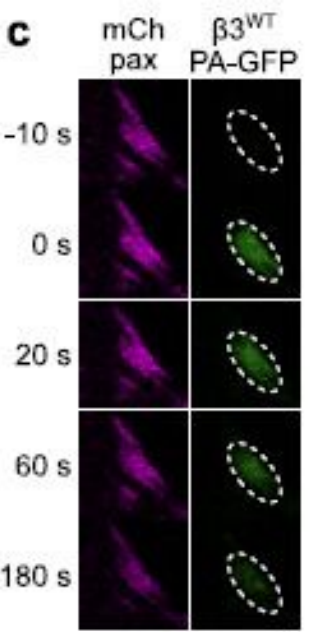

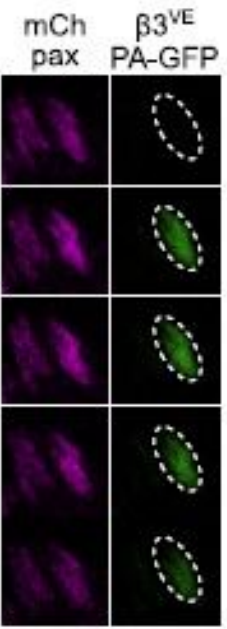

$\mathrm{mCh} \quad \beta 3^{\mathrm{VE} / \mathrm{A}}$

pax PA-GFP

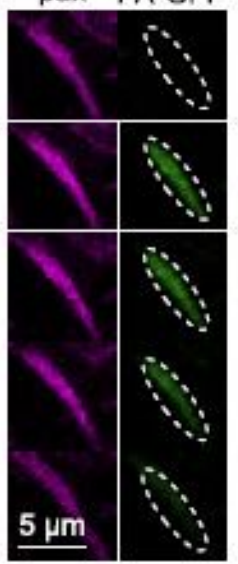

f

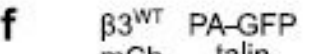

$\beta 3^{\mathrm{VE}}$ PA-GFP

$\beta 3^{\text {VENA }}$ PA-GFP
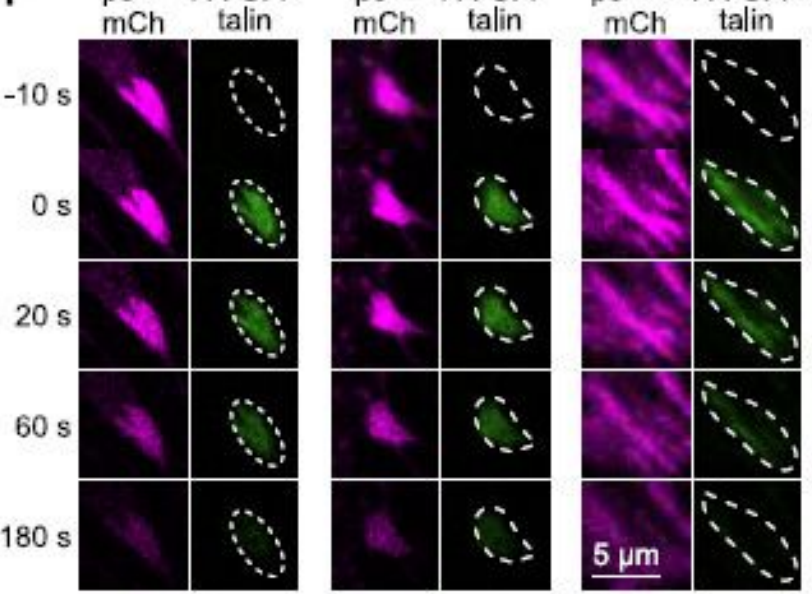

i
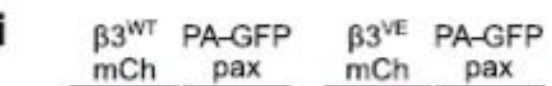

$\beta 3^{\text {VENA }}$ PA-GFP
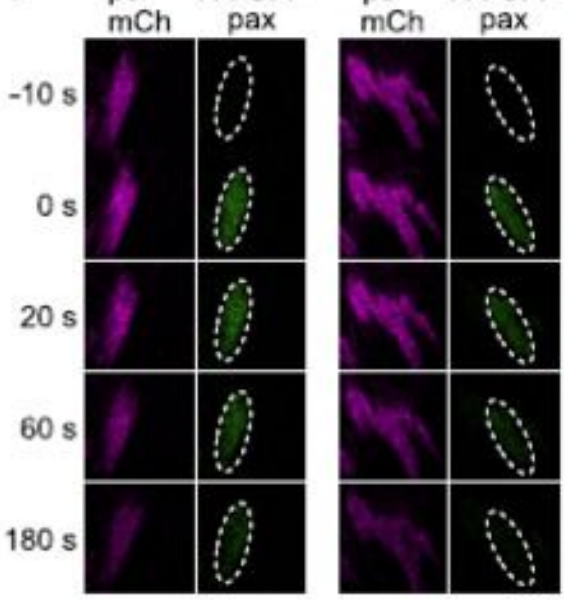

Figure 2

Analysis of the dissociation dynamics of the $\beta 3$ integrin/talin/paxillin complex. $(a, d, g)$ Experimental dissociation curves of (a) B3_PA-GFP integrins from mCherry_paxillin-positive focal adhesions and of (d) PA-GFP_talin and (g) PA-GFP_paxillin from $\beta 3$ _mCherry-positive focal adhesions. (b,e,h) Box plot of the half-lives of (b) B3_PA-GFP integrins in mCherry_paxillin-positive FAs and of (e) PA-GFP_talin and (h) PA- 
GFP_paxillin in 33 _mCherry-positive FAs. Statisticalanalysis is provided in Supplementary Table 3. (c,f,i) Representative images of photoactivation time series of experiments in a-b, d-e and f-g.
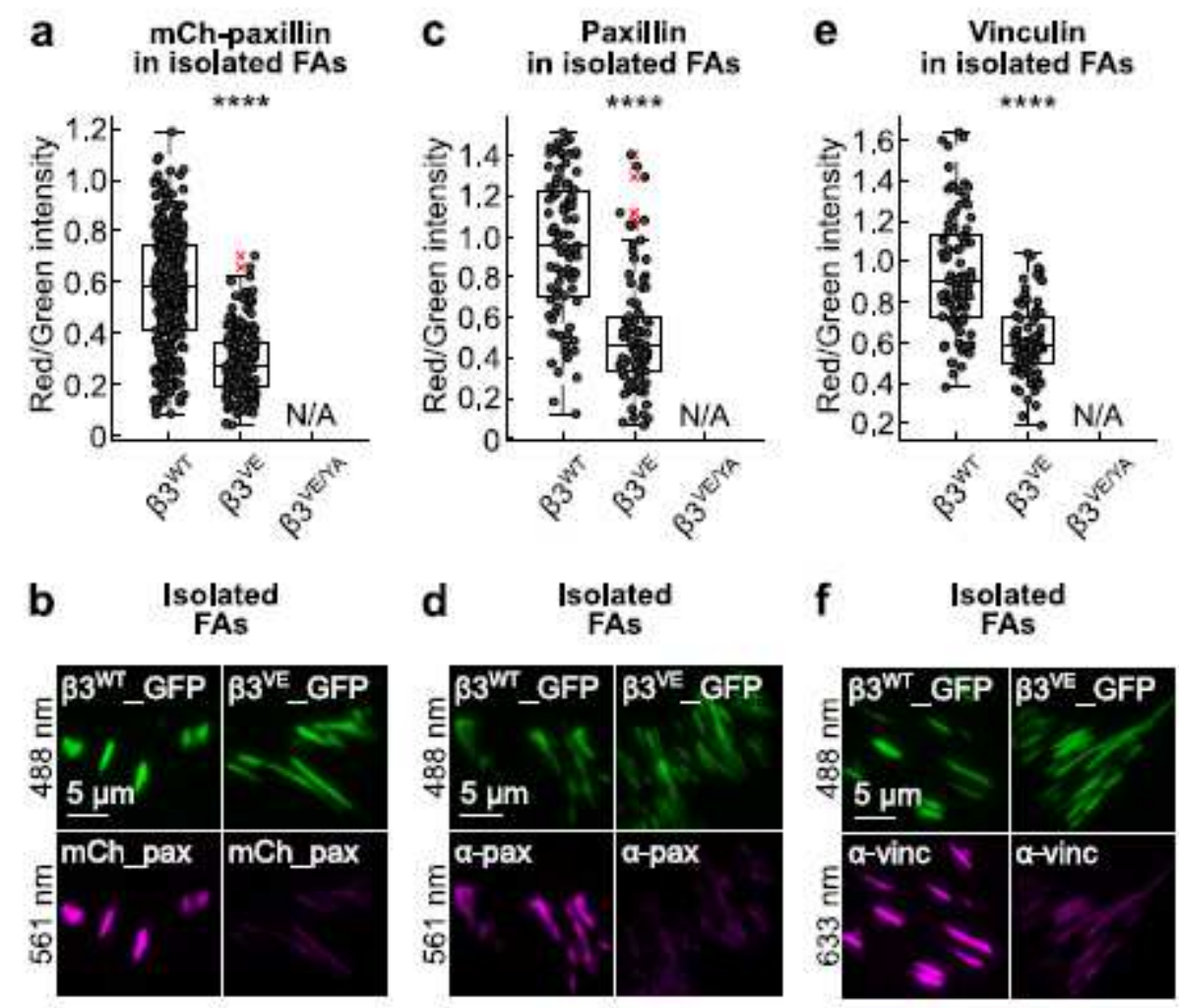

\section{Figure 3}

Analysis of paxillin and vinculin binding upon isolation of FA-complexes. $(a, c, e)$ Quantification of cytoplasmic proteins retained in $\beta 3$ WT_GFP- or $\beta 3$ VE_GFP-positive FAs, isolated from NIH-3T3 fibroblasts. (a) mCherry_paxillin, (c) endogenous paxillin and (e) endogenous vinculin. Statistical analysis is provided in Supplementary Table 4. (b,d,f) Representative TIRF images of FAs isolated from NIH-3T3 cells of experiments quantified in $a, c$ and $e$. 

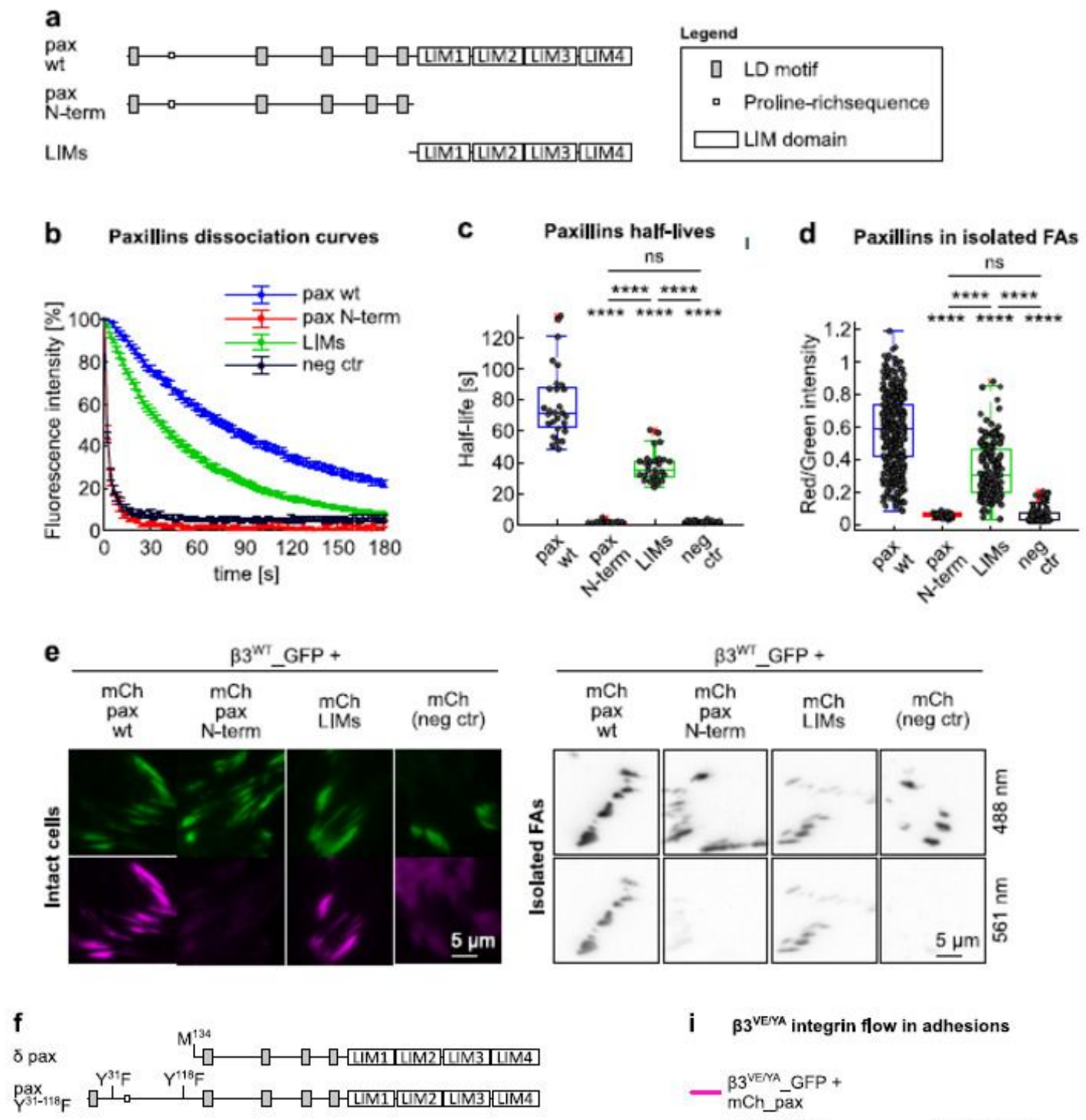

i $\beta 3^{\text {VEYA }}$ integrin flow in adhesions
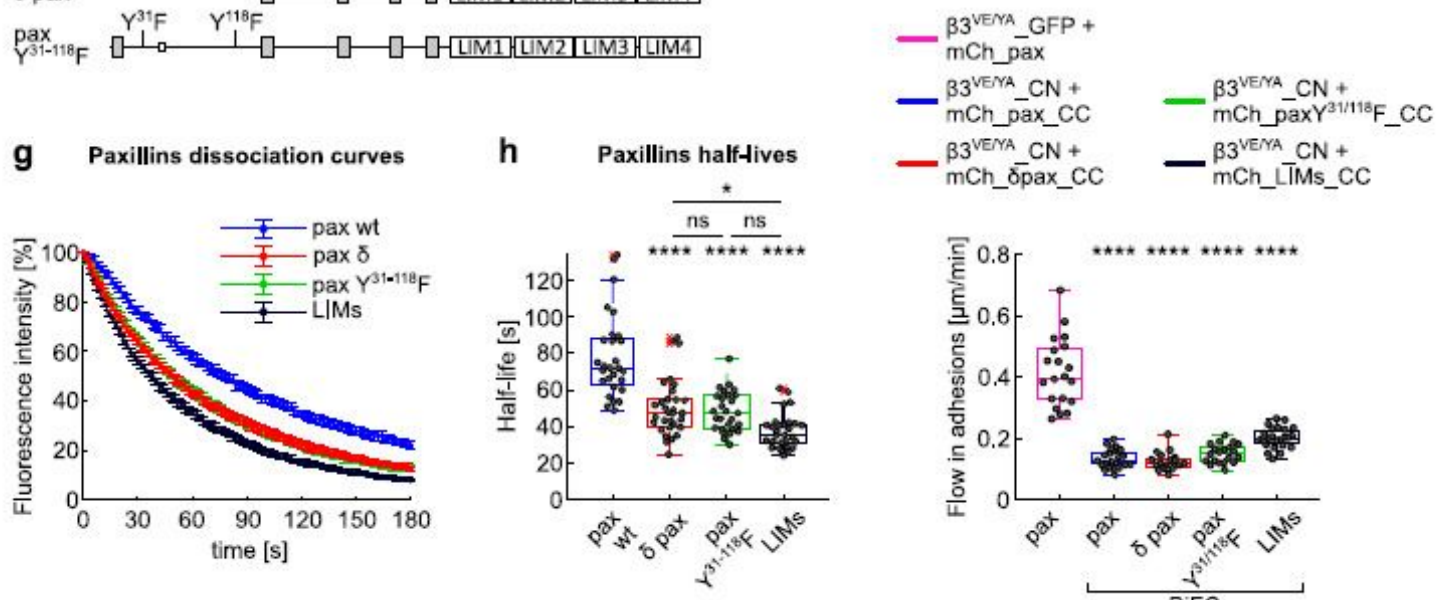

\section{Figure 4}

Paxillin binding to adhesions is driven by the LIM domains but stabilized by the N-terminus. $(a, f)$ Schematic representation of paxillin (a) truncation mutants and (f) N-terminal mutants. All proteins were $\mathrm{N}$-terminally tagged with PA-GFP or mCherry. $(b, g)$ Experimental dissociation curves of PA-GFP_paxillin wt and (b) truncation mutants or (g) N-terminal mutants, from $\beta 3$ _mCherry-positive FAs. (c,h) Box plot of the half-life of PA-GFP_paxillin wt and (c) truncation mutants or (h) N-terminal mutants, in $\beta 3$ _mCherry- 
positive FAs. Statistical analysis is provided in Supplementary Table 3. (d) Quantification of mCherry_paxillin wt and truncation mutants retained in FAs, isolated from NIH-3T3 cells co-expressing B3WT_GFP. Statistical analysis is provided in Supplementary Table 4. (e) Representative TIRF images of FAs in NIH-3T3 cells co-expressing $\beta 3$ WT_GFP and mCherry_paxillin proteins. Left panel: FAs in intact cells; right panel: isolated FAs. (i) Quantification of the $\beta 3 V E / Y A$ integrin flow in adhesions, expressed as mean displacement over time $(\mu \mathrm{m} / \mathrm{min})$ per replicate. Statistical analysis is provided in Supplementary Table 2. Neg ctr: PA-GFP in photoactivation experiments and mCherry in isolated FAs.

a

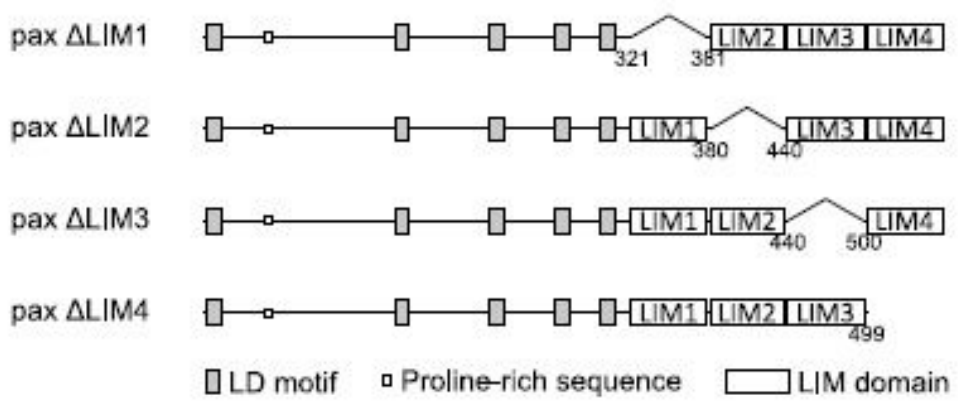

b

UM1 $\mathrm{G}^{322}$ VCGACKKPIAGQVVTAMGKTWHPEHFVCTHCQEEIGSRNFFERDGQPYCEKDYHNLFS ${ }^{330}$ UIM2 P $^{391}$ RCYYCNGPILDKVVTALDRTWHPEHFECAQCGAFEGPEGEHEKDGKAYCRKDYFDMFA ${ }^{439}$ LIM3 $\mathrm{P}^{440}$ KCGGCARAILENYI SALNTLWHPECFVCRECFT'PFVNGSFFEHDGQPYCEVVHYHERRG $^{498}$ LIM4 S $^{499}{ }^{2}$ CSGCQKPI TGRCI TAMAKKFHPEHFVCAFCLKQLNKGTEKEQNDKPYCONCFLKLIFC ${ }^{557}$

d Paxillins dissociation curves

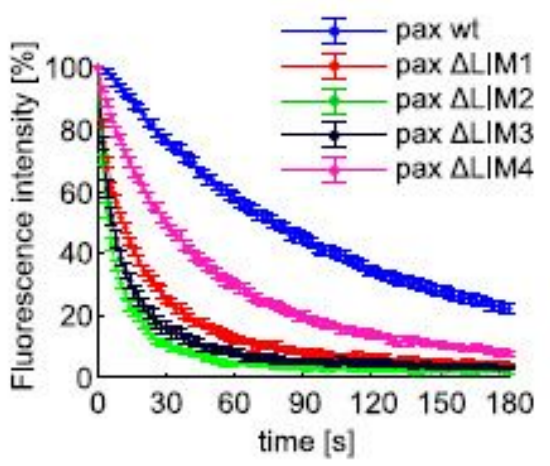

g

g$$
\beta 3^{\text {WT }} \text { GFP + }
$$

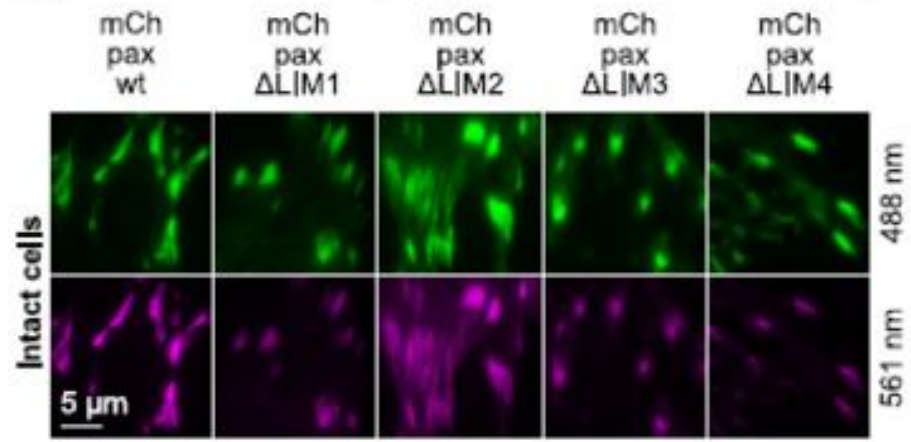

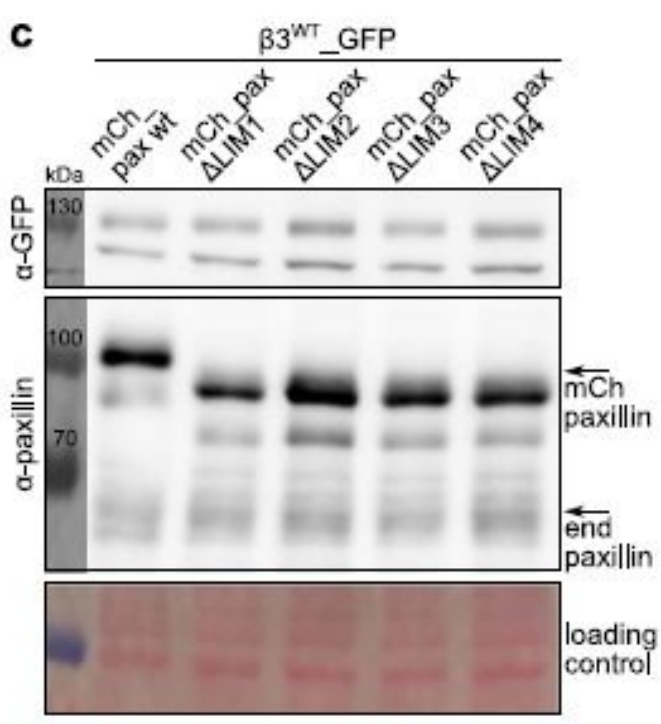
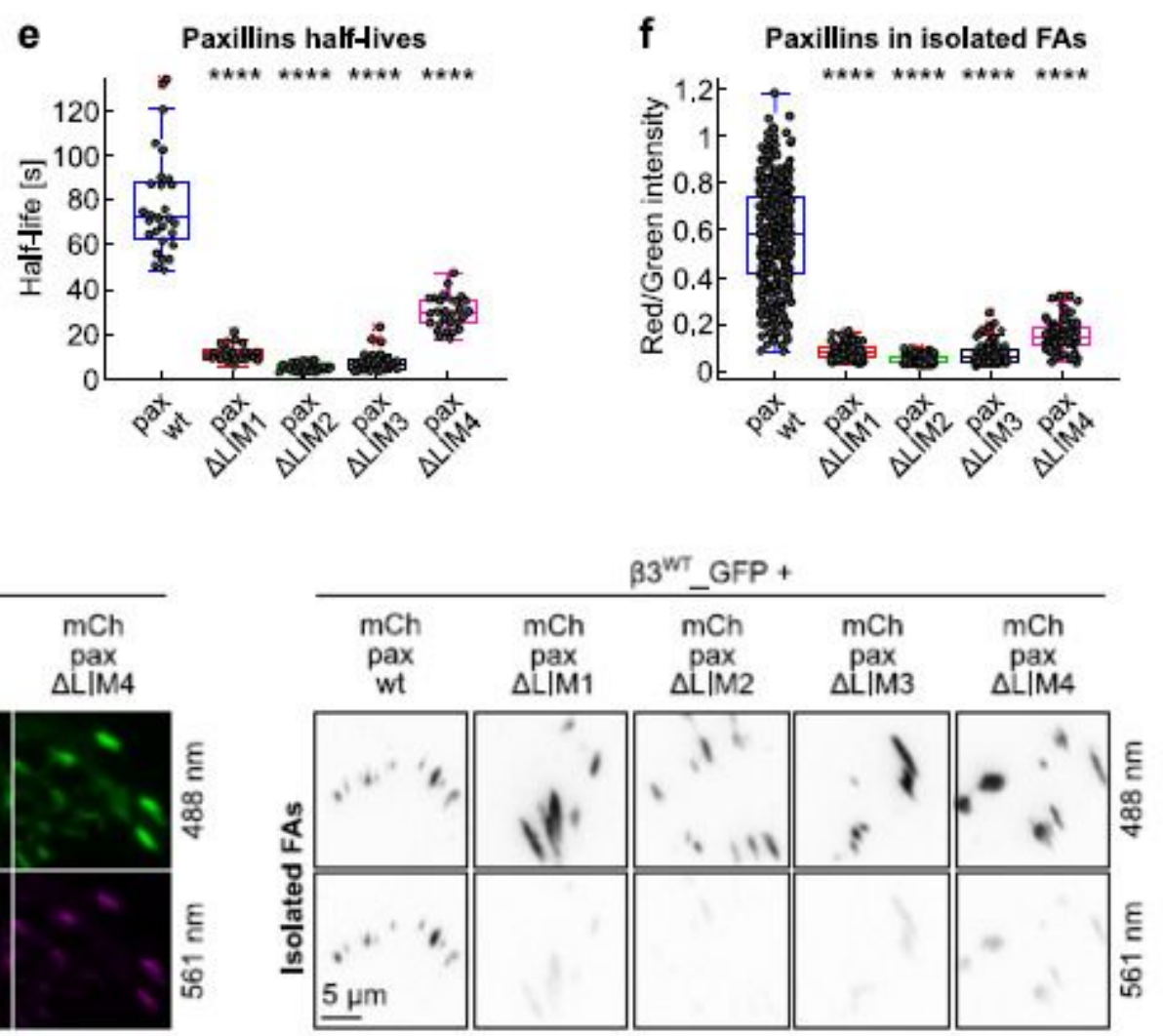

Figure 5 
LIM domain deletion analysis by photoactivation and FA-isolation. (a) Schematic representation of the paxillin LIM domain deletion mutants. All proteins were N-terminally tagged with PA-GFP or mCherry. (b) Alignment of the four paxillin LIM domains. Grey: zinc-binding residues. (c) Western blot of protein extracts from NIH-3T3 fibroblasts transiently co-expressing $\beta 3$ WT_GFP and mCherry-tagged paxillin proteins, wt or deletion mutants. Loading control: Ponceau staining. (d) Experimental dissociation curves of PA-GFP_paxillin wt and deletion mutants from B3WT_mCherry-positive FAs. (e) Box plot of the corresponding half-lives Statistical analysis is provided in Supplementary Table 3. (f) Quantification of mCherry_paxillin wt and mutants retained in FAs isolated from NIH-3T3 cells co-expressing B3WT_GFP. Statistical analysis is provided in Supplementary Table 4. (g) Representative TIRF images of FAs in $\mathrm{NIH}-$ 3T3 cells co-expressing $\beta 3$ WT_GFP and mCherry_paxillin proteins. Left panel: FAs in intact cells; right panel: isolated FAs. 


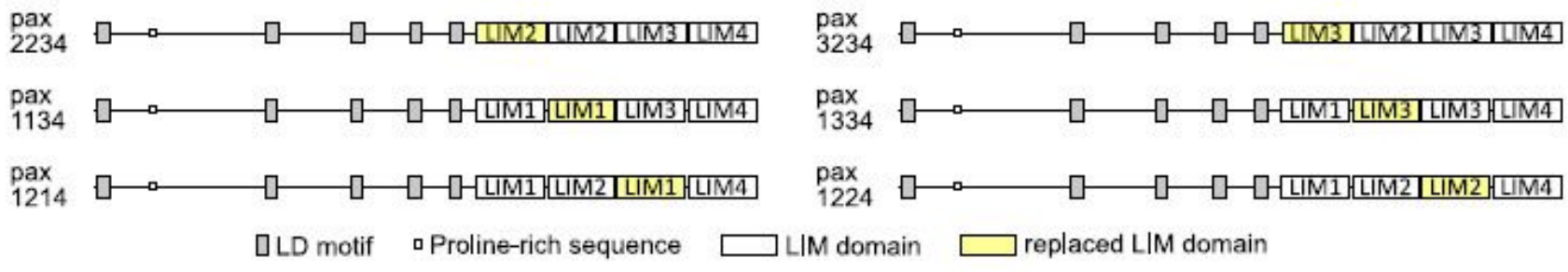

b Paxillins dissociation curves

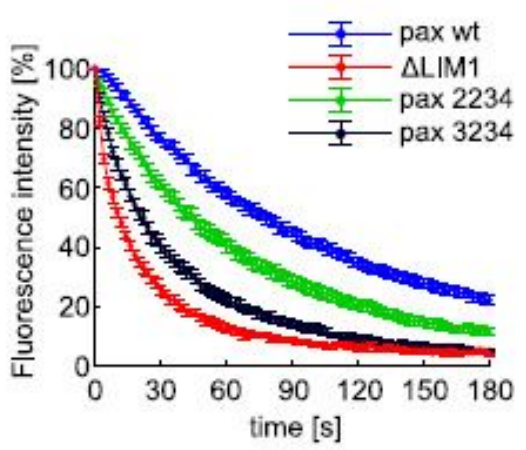

C Paxillins half-lives

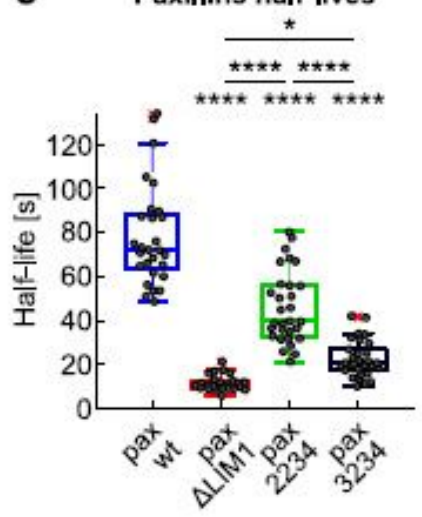

d Paxillins dissociation curves

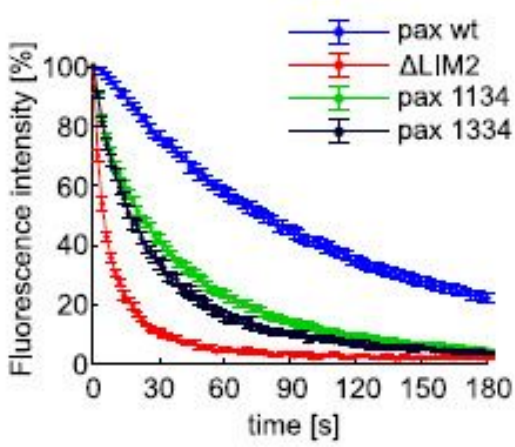

e

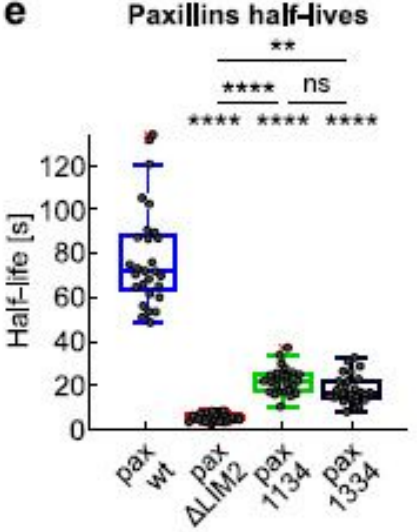

f Paxillins dissociation curves
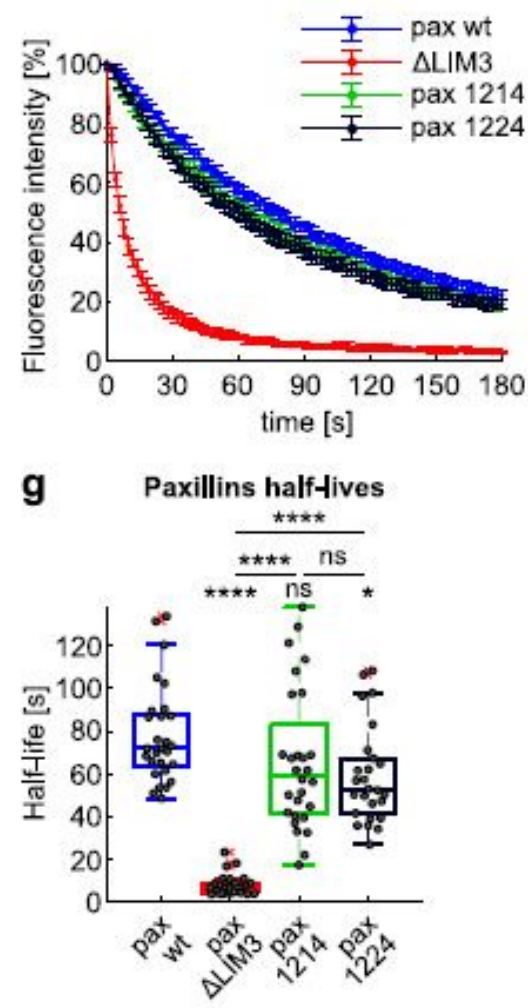
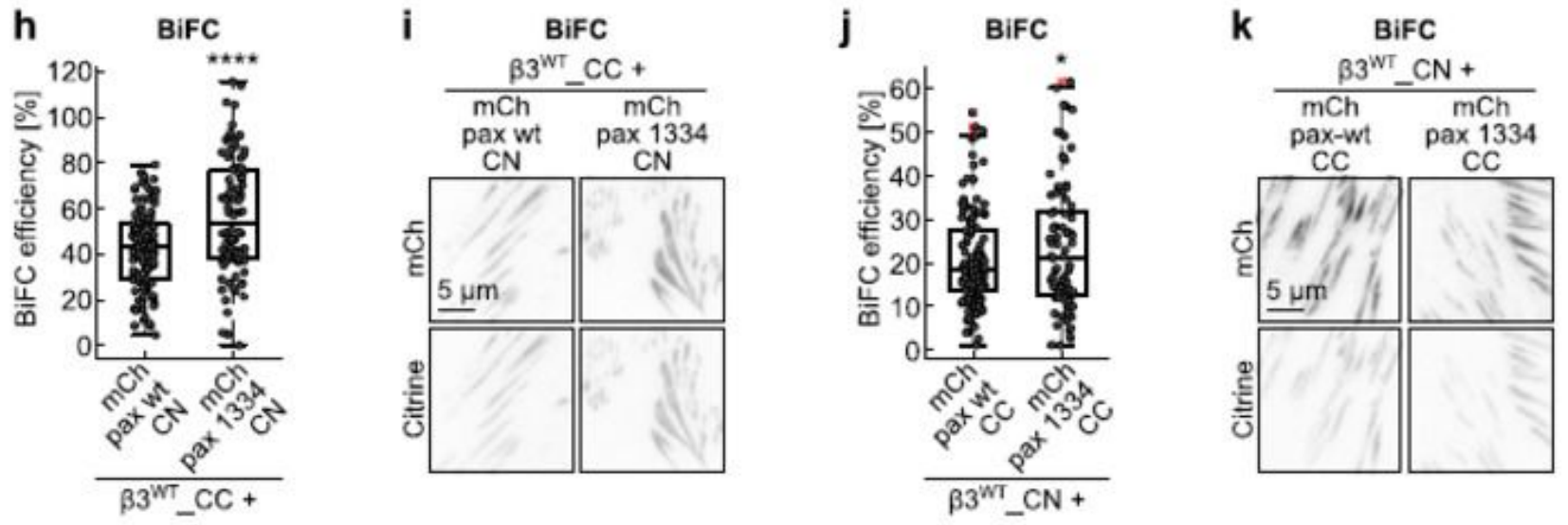

\section{Figure 6}

LIM domain-specific functions assessed by the LIM domain replacement strategy. (a) Schematic representation of the paxillin mutants obtained by the LIM domain replacement strategy. From the top line: replacement of LIM1, replacement of LIM2 and replacement of LIM3. All proteins were N-terminally tagged with PA-GFP. $(b, d, f)$ Experimental dissociation curves of PA- GFP_paxillin wt and (b) LIM1 mutants, (d) LIM2 mutants and (f) LIM3 mutants, from $\beta 3$ _mCherry-positive FAs. $(\mathrm{c}, \mathrm{e}, \mathrm{g})$ Box plot of the half-lives 
of PA-GFP_paxillin wt and (c) LIM1 mutants, (e) LIM2 mutants and (g) LIM3 mutants in 33 _mCherrypositive FAs. Statistical analysis is provided inSupplementary Table 3. (h,i) BiFC upon co-expression of mCherry_paxillin_CN or its corresponding paxillin 1334 mutant and $\beta 3 \mathrm{WT}$ integrin C-terminally tagged with the citrine $\mathrm{C}$-terminal fragment in NIH-3T3 cells. (h) Quantification of the BiFC efficiency. Satistical analysis is provided in Supplementary Table 1. (i) Representative TIRF images. (j,k) BiFC upon coexpression of mCherry_paxillin_CC or its corresponding paxillin 1334 mutant and $\beta 3 W T$ integrin Cterminally tagged with the citrine N-terminal fragment in NIH-3T3 cells. (j) Quantification of the BiFC efficiency. Statistical analysis is provided in Supplementary Table 1. (k) Representative TIRF images.

a Conservation of the paxillin LIM4 domain across species

R. norvegicus

M. musculus

PXN

CSGCQKPITGRCITAMAKKFHPEHFVCAFCLKQLNKGTFKEQNDKPYCONCFLKLFC

PXN

CSGCQKPITGRCITAMAKKEHPEHFVCAFCLKQLNKGTFKEQNDKPYCQSCFLKLFC

CSGCQKPITGRCITAMAKKEHPEHFVCAFCLKQLNKGTEKEQNDKPYCQSCFVKLFC

G. gallus

CSGCQKPITGRCITAMGKKFHPEHFVCAFCLKQLNKGTEKEQNDKPYCQNCFLKLFC

X. laevis

$\operatorname{PXN}$

CSCCQKPITGRCITAMGKKFHP HEVCAFCLKQLNKGTEKEQNDKPYCQNCEVKLEC

D. rerio

PXNA

D. melanogaster Pax

C. elegans

$\mathrm{PXL1}$

D. discoideum

PAXB

CSGCQKPITGRCITAMGKKEHPEHFVCAFCLKQLNKGTEKEQNDKPYCQSCFVKLFS
CAGCSKPITGRCITAMEKKEHPEHFVCAFCLKQLNKGTEKEQKDKPYCHTCFDKIFG

CSQCRGAINGRCVAAMGRKEHPEHERCSYCNHQLTKGTEKEVDRRP FCHKCYNNTYALTPA

CSCCGKAVSGRCVDALDKKWHPEHFVCAFCMNPI AGGSYTANNGKPYCKGCHNKLFA

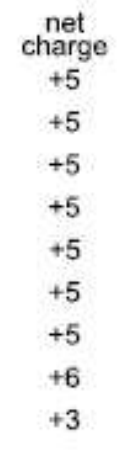

LIM4 triple mutant

CSGCOKPITGQCITAMAKKFḦPEHFVCAFCLIEQLNKGTFKEQNDKPYCQNCELDLEC

0

b
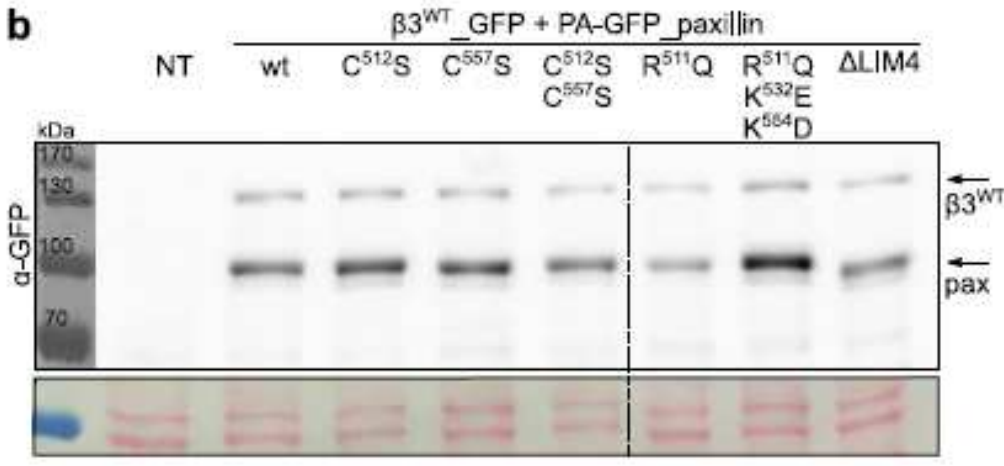

d

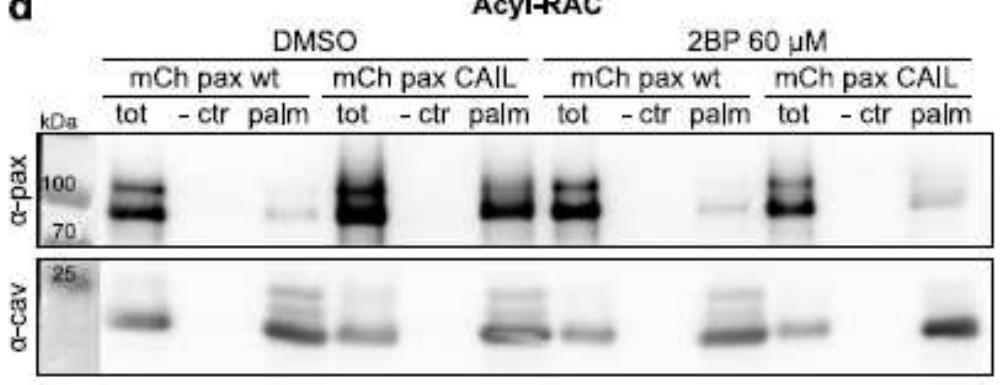

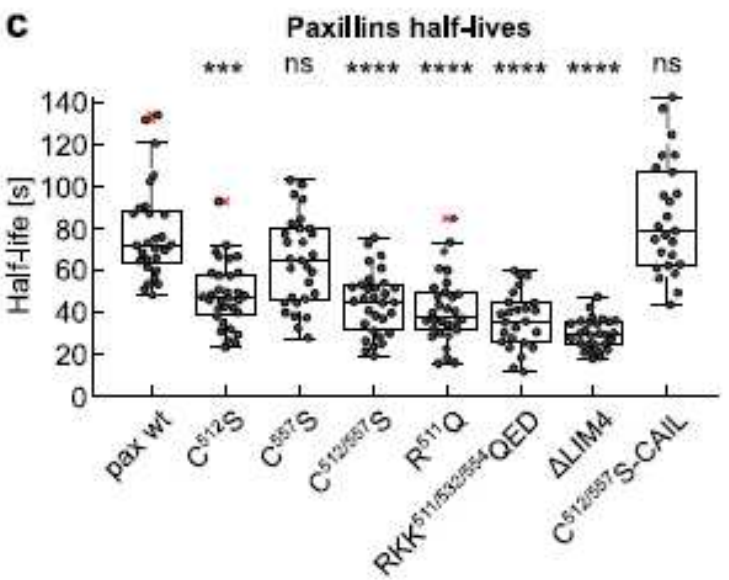

\section{Figure 7}

Paxillin LIM4 domain carries membrane binding features relevant for FA binding. (a) Alignment of the amino acid sequence of paxillin LIM4 across species and triple human paxillin LIM4 mutant (bottom). The latter was obtained by replacing three basic amino acids with neutral or acidic residues found at the corresponding positions in other paxillin LIM domains (RKK511/532/554QED). Grey: zinc-binding 
residues; blue: positive residues; red: negative residues; bold: non-conserved residues in respect to the human paxillin LIM4 domain; arrows indicate the conserved free cysteines; yellow: conserved GRC motif. (b) Expression analysis, via western blot, of the paxillin LIM4 mutants. Loading control: Ponceau staining. (c) Box plot of the half-lives of PA-4GFP_paxillin wt and LIM4 mutants in 33 WT_mCherry-positive FAs. Statistical analysis is provided in Supplementary Table 3. (d) Acyl-Resin Assisted Capture assay from cell transiently transfected with mCherry_paxillin wt or mCherry_paxillin-CAIL, untreated (DMSO) or treated with 2-bromopalmitate. NT: not transfected; tot: total cell lysate; - ctr: negative control (tris-base); palm: fraction of palmitoylated proteins (hydroxylamine); 2BP: 2-bromopalmitate. 

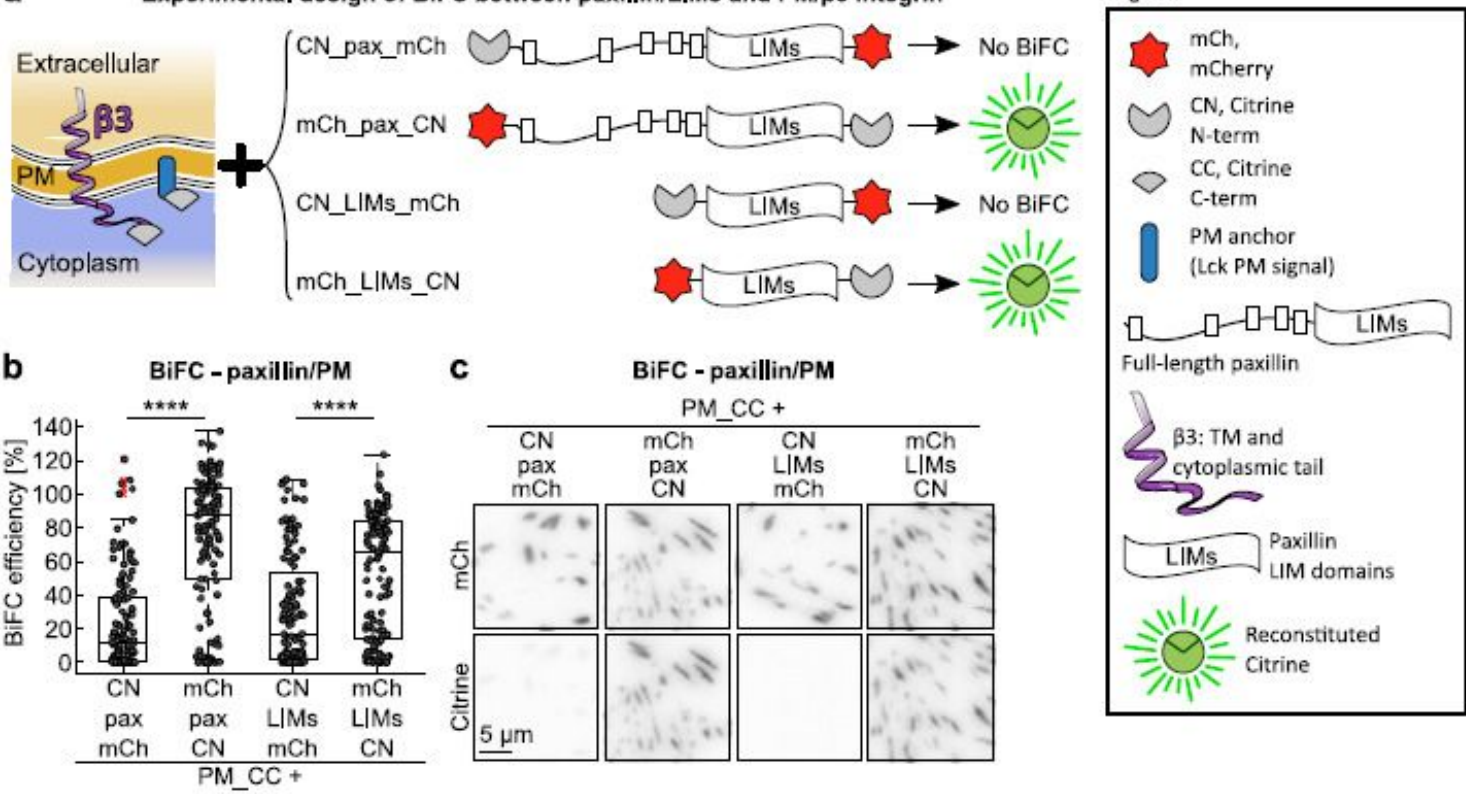

d BiFC - paxillin/ß33 integrin
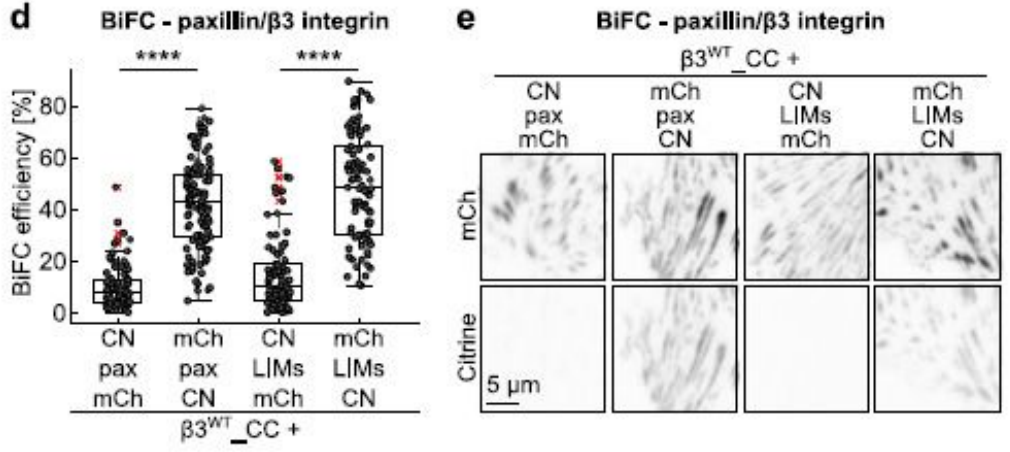

f

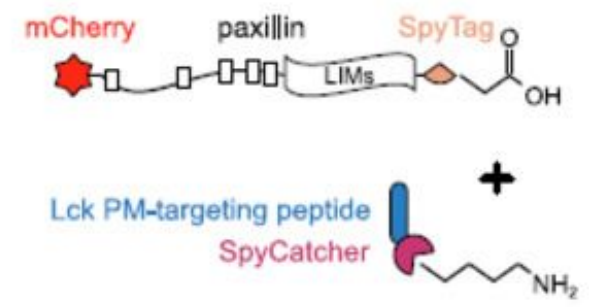

Spontaneous isopeptide bond formation

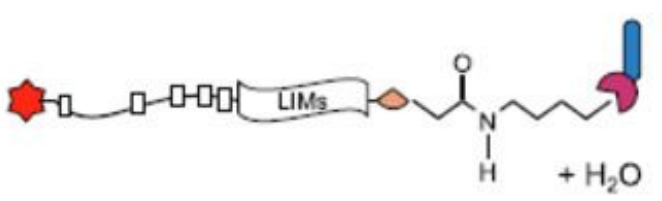

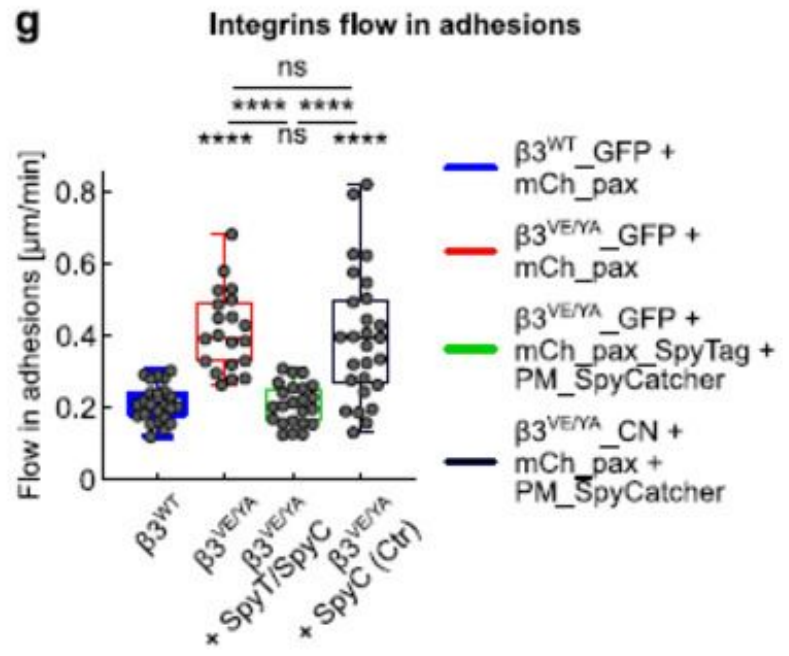

Figure 8

Preferential orientation of the paxillin LIM domains within FAs. (a) Overview of the molecules tested with the BiFC assays and a summary outcome, in terms of citrine fluorescence, of their pairwise expression. $(b, d)$ Quantification of the BiFC signal generated by the co-expression of each of the paxillin constructs shown in figure a with (b) the plasma membrane-localized CC fragment, in Swiss-3T3 or with (d) the $\beta 3$ WT C-terminally tagged with CC, in NIH-3T3 cells. Statistical analysis is provided in Supplementary 
Table 1. (c,e) Representative TIRF images of experiments quantified in b and d. (f) Schematic representation of the functioning SpyTag/SpyCatcher technology 64 and of how it has been used in our cellular system. (g) Quantification of the integrins flow in adhesions, expressed as mean displacement over time $(\mu \mathrm{m} / \mathrm{min})$ per replicate. Statistical analysis is provided in Supplementary Table 2.

\section{Supplementary Files}

This is a list of supplementary files associated with this preprint. Click to download.

- Movie1.avi

- Movie2.avi

- Movie3.avi

- SupplementaryTables14.xlsx

- FigS1final.jpg

- FigS2final.jpg

- FigS3final.jpg

- FigS4final.jpg 